\title{
Meroterpenoids: A Comprehensive Update Insight on Structural Diversity and Biology ${ }^{\dagger}$
}

\author{
Mamona Nazir 1,*, Muhammad Saleem ${ }^{2}$ (D), Muhammad Imran Tousif ${ }^{3}$ (D), Muhammad Aijaz Anwar ${ }^{4}$, \\ Frank Surup ${ }^{5}(\mathbb{D})$, Iftikhar Ali ${ }^{6,7}\left(\mathbb{D}\right.$, Daijie Wang ${ }^{6}$, Nilufar Z. Mamadalieva ${ }^{8,9}\left(\mathbb{D}\right.$, Elham Alshammari ${ }^{10}(\mathbb{D}$, \\ Mohamed L. Ashour 11,12 (D), Ahmed M. Ashour ${ }^{13}\left(\mathbb{D}\right.$, Ishtiaq Ahmed ${ }^{14, *}$, Elizbit ${ }^{15}$, Ivan R. Green ${ }^{16}(\mathbb{D}$ \\ and Hidayat Hussain $8, *$ (D)
}

check for updates

Citation: Nazir, M.; Saleem, M.; Tousif, M.I.; Anwar, M.A.; Surup, F.; Ali, I.; Wang, D.; Mamadalieva, N.Z.; Alshammari, E.; Ashour, M.L.; et al. Meroterpenoids: A Comprehensive Update Insight on Structural Diversity and Biology . Biomolecules 2021, 11, 957. https://doi.org/ 10.3390/biom11070957

Received: 4 June 2021

Accepted: 24 June 2021

Published: 29 June 2021

Publisher's Note: MDPI stays neutral with regard to jurisdictional claims in published maps and institutional affiliations.

Copyright: (c) 2021 by the authors. Licensee MDPI, Basel, Switzerland. This article is an open access article distributed under the terms and conditions of the Creative Commons Attribution (CC BY) license (https:/ / creativecommons.org/licenses/by/ $4.0 /)$.
1 Department of Chemistry, Government Sadiq College Women University Bahawalpur, Bahawalpur 63100, Pakistan

2 Institute of Chemistry, Baghdad-ul-Jadeed Campus, The Islamia University of Bahawalpur, Bahawalpur 63100, Pakistan; m.saleem@iub.edu.pk

3 Department of Chemistry, DG Khan Campus, University of Education Lahore, Dera Ghazi Khan 32200, Pakistan; imran.tousif@ue.edu.pk

4 Pharmaceutical Research Division, PCSIR Laboratories Complex Karachi, Karachi 75280, Pakistan; maanwarpk@yahoo.com

5 Microbial Drugs, Helmholtz Centre for Infection Research, 38124 Braunschweig, Germany; Frank.Surup@helmholtz-hzi.de

6 School of Pharmaceutical Sciences and Key Laboratory for Applied Technology of Sophisticated Analytical Instruments of Shandong Province, Shandong Analysis and Test Center, School of Pharmaceutical Sciences, Qilu University of Technology (Shandong Academy of Sciences), Jinan 250014, China; iftikhar.ali@kiu.edu.pk (I.A.); wangdaijie@qlu.edu.cn (D.W.)

7 Department of Chemistry, Karakoram International University, Gilgit 15100, Pakistan

8 Department of Bioorganic Chemistry, Leibniz Institute of Plant Biochemistry, Weinberg 3, D-06120 Halle, Germany; nmamadalieva@yahoo.com

9 Institute of the Chemistry of Plant Substances, Uzbekistan Academy of Sciences, Mirzo Ulugbek Str 77, Tashkent 100170, Uzbekistan

10 Department of Pharmacy Practice, College of Pharmacy, Princess Nourah Bint Abdulrahman University, Riyadh 11671, Saudi Arabia; ejalshammari@pnu.edu.sa

11 Department of Pharmaceutical Sciences, Pharmacy Program, Batterjee Medical College, Jeddah 21442, Saudi Arabia; mohamed.ashour@bmc.edu.sa or ashour@pharma.asu.edu.eg

12 Department of Pharmacognosy, Faculty of Pharmacy, Ain Shams University, Cairo 11566, Egypt

13 Department of Pharmacology and Toxicology, College of Pharmacy, Umm Al-Qura University, P.O. Box 13578, Makkah 21955, Saudi Arabia; amashour@uqu.edu.sa

14 Department of Chemical Engineering and Biotechnology, University of Cambridge, Cambridge CB2 1TN, UK

15 Department of Materials Engineering, National University of Sciences and Technology (NUST) H12, Islamabad 44000, Pakistan; malkaalizachem@gmail.com

16 Department of Chemistry and Polymer Science, University of Stellenbosch, Private Bag X1, Matieland, Stellenbosch 7600, South Africa; irg@sun.ac.za

* Correspondence: mamonanazir.de@gscwu.edu.pk or mamonanazir.de@gmail.com (M.N.); ishtiaq.chem@googlemail.com (I.A.); Hidayat.Hussain@ipb-halle.de or hussainchem3@gmail.com (H.H.)

+ This article is dedicated to Prof. Dr. Ludger Wessjohann on his 60th birthday.

Abstract: Meroterpenoids are secondary metabolites formed due to mixed biosynthetic pathways which are produced in part from a terpenoid co-substrate. These mixed biosynthetically hybrid compounds are widely produced by bacteria, algae, plants, and animals. Notably amazing chemical diversity is generated among meroterpenoids via a combination of terpenoid scaffolds with polyketides, alkaloids, phenols, and amino acids. This review deals with the isolation, chemical diversity, and biological effects of 452 new meroterpenoids reported from natural sources from January 2016 to December 2020. Most of the meroterpenoids possess antimicrobial, cytotoxic, antioxidant, anti-inflammatory, antiviral, enzyme inhibitory, and immunosupressive effects.

Keywords: meroterpenoids; structural diversity; antidiabetic; anticancer; antioxidant; anti-inflammatory 


\section{Introduction}

Natural products and their analogs in today's age play a crucial role in the development of novel drugs because of their tremendous structural diversity [1-5]. It has been reported that out of the 877 New Chemical Entities (NCEs) established between 1981 and 2002, ca. 49\% arose from natural products, or, synthetic molecules based on natural-product-pharmacophores [6,7]. However, pharmaceutical research into natural secondary metabolites has declined in the last two decades because of the difficulty in isolating compounds with skeletally novel frameworks from natural resources rather than from combinatorial synthetic protocols [7-9].

The term "meroterpenoid" was first used by Cornforth [10] in 1986 to describe natural products bearing a mixed terpenoid biogenesis. The prefix "mero-" is derived from the Greek word "merus" which means "fragment, or part, or partial" [11-13]. Meroterpenoids are thus a class of natural products derived from hybrid polyketide or non-polyketide and terpenoid biosynthesis. The unusual enzyme reactions responsible for connectivities among their structures, and their unique ring cores create a most interesting chemical diversity among meroterpenoids $[11,14,15]$. Interestingly, meroterpenoids are mostly reported from fungi and marine organisms while only a limited number of meroterpenoids were obtained from plants [9].

Ubiquinone-10, $\alpha$-tocopherol and the anticancer drugs, viz., vinblastine and vincristine are included in the meroterpenoid classification [12,13]. Due to the interesting chemical diversity, meroterpenoids illustrated an impressive range of biological effects, viz., antioxidant [16-18], anti-inflammatory [19-22], anti-atherosclerotic [23], anti-adipogenic [24], anti-diabetic [25], anti-carcinogenic [26,27], and neuroprotective [28] activities. In 2009, Geris and Simpson [12] published a review on fungal meroterpenoids. Peng and Qiu [13] reported on 135 meroterpenoids which were all obtained from the fungal genus Ganoderma sp. Quite recently, another four reviews appeared: (i) El-Demerdash et al. [29] summarized 320 meroterpenoids from marine fungi covering from 2009 to 2019. (ii) Zhao et al. [30] focused on terrestrial and marine-derived fungal 709 meroterpenoids (covered 2009-2020). (iii) Gozari et al. [31] reviewed only 190 meroterpenoids from bacteria and fungi that showed significant preclinical effects. (iv) Jiang et al. [32] summarized the described 1585 fungal meroterpenoids in 2009-2019.

Our review article describes the systematic and complete summary of potentially bioactive meroterpenoids from all natural sources except fungal meroterpenoids during the last five years 2016-2020 (January 2016 to December 2020). In point of fact, an amazing 452 new meroterpenoids were discovered during this period, which were mostly tested for their various biological activities.

\section{Meroterpenoid Classification}

The structures of meroterpenoids are exceptionally diverse and complex which is why classifications of these compounds are not easy. Meroterpenoids were often classified in two ways. The first way is to classify these compounds as polyketide- and non-polyketideterpenoids previously described by Geris and Simpson [12]. The second way is to classify these compounds based on a common scaffold, common natural product skeleton, or heterocyclic ring system, viz., phloroglucinol, syncarpic acid, sesquiterpene, phthalide, benzofuran and phenylfuran [11,12]. We followed the second method.

\section{Phloroglucinol-Based Meroterpenoids}

Among other anticancer meroterpenoids, diformylphloroglucinol-derived meroterpenoids from Psidium guajava $\mathrm{L}$. were identified by spectroscopic analyses and ECD calculations as psiguajavadials A (1) and B (2), and guajadial (3) (Figure 1) [33]. All these metabolites showed antitumor activity against HCT116, Huh7, DU145, CCRF-CEM, and A549 cells. (Table 1). Compounds 1 and 2 displayed dose-dependent inhibition of Top1 activity [33]. Guajadial (3), inhibited endothelial cell proliferation and migration as well as suppressing tumor growth in human NSCLC (A549 and H1650 cells) and xenograft 
mouse models. This potential has been reported as being a significant antineoplasmic activity of 3. The Western blotting method to study the underlying mechanisms of VEGF receptor (VEGFR)2-mediated revealed that compound 3 inhibited A549 $\left(\mathrm{IC}_{50}=3.58 \mu \mathrm{M}\right)$ proliferation via blocking the Ras/MAPK pathway [34]; this activity of 3 is higher than the potential of cisplatin $\left(\mathrm{IC}_{50}\right.$ value of 7.51$)$, which was used as a positive control.

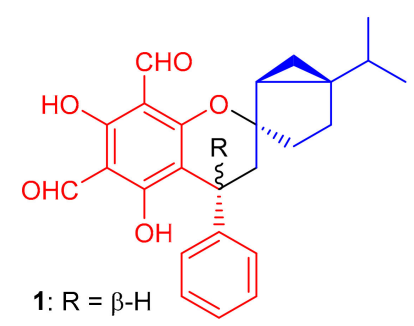

(

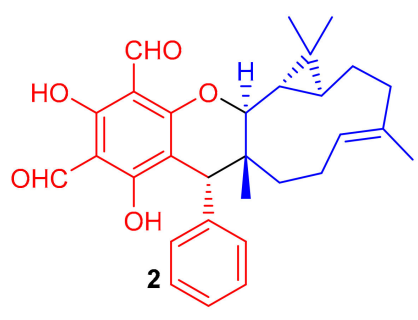

3: $\mathrm{R}_{1}=\beta-\mathrm{H}$,

$\mathrm{R}_{2}=\alpha-\mathrm{H}$,

$\mathrm{R}_{3}=\beta-\mathrm{Me}$

Figure 1. Structures of phloroglucinol-based meroterpenoids 1-3.

Diformylphloroglucinol-based meroterpenoids, viz., guajavadials A-C (4-6) (Figure 2) were obtained from P. guajava and also exhibited cytotoxicity against A-549, HL-60, MCF-7, SMMC-7721, and SW480 cancer cell lines with $\mathrm{IC}_{50}$ values between 2.28-3.38 $\mu \mathrm{M}$ (Table 1). Compound 6 displayed the highest potential $\left(\mathrm{IC}_{50}=3.54 \mu \mathrm{M}\right)$ against SMMC-7721 cell lines which is higher than the standard cisplatin $\left(\mathrm{IC}_{50}=19.8 \mu \mathrm{M}\right)$ [35]. The structures and activity differences revealed that the arrangement of the isoprene units is responsible for the activity potential, and thus the terpenoidal skeleton indeed plays a significant role in the activity level, as can be seen in compounds 5 and 6 [35].

Shang et al. [36] isolated a small range of cytotoxic formylphloroglucinol-derived meroterpenoids; the eucalrobusones A-I (7-15) (Figure 2) from Eucalyptus robusta. In the MTT assay, compound 7 moderately inhibited the growth of HepG2 ( $\left.\mathrm{IC}_{50}: 18.5 \mu \mathrm{M}\right)$ and U2OS ( $\mathrm{IC}_{50}: 45.0 \mu \mathrm{M}$ ), while metabolite 10 possesses a weaker potential against HepG2 $(26.7 \mu \mathrm{M})$ (Table 1). Compound 15 only exhibited moderate inhibition of U2OS cell lines with an $\mathrm{IC}_{50}$ value of $42.25 \mu \mathrm{M}$. Only metabolite 9 proved to be the most potent anticancer agent against the three target cancer cells with $\mathrm{IC}_{50}$ : of 7.40 to 8.99 . Activity of compound 9 has been reported to be comparable to doxorubicin $\left(\mathrm{IC}_{50}=5.23,2.66\right.$ and $1.14 \mu \mathrm{M}$, respectively). A study on the mechanism of action of compound 9 revealed that it significantly inhibited cell division exerting cell proliferation on MCF-7 in a concentration dependent manner. Eucalrobusones A (7) and B (8) bearing an unusual skeleton having a maaliane sesquiterpene core is linked to a phloroglucinol unit. On the other hand, the chroman ring in eucalrobusone $\mathrm{E}$ (11) is attached to a bicyclogermacrane unit at the C3/C-4 position. Meroterpenoid 12 bearing a phloroglucinol unit is linked to an aromatic dehydromenthane monoterpene group. Eucalrobusones G-I (13-15) are cubebane-based phloroglucinol-based meroterpenoids linked through a 1-oxaspiro[5.5] undecane unit [36].

Compounds (16-20) (Figure 3) were obtained from Eugenia umbelliflora belonging to the Myrtaceae family and tested for cytotoxic effects towards murine melanoma cells (B16F10 cell) and the leukemic cells (Nalm-6 cells and k562) (Table 1). Interestingly, compound 18 displayed the highest cytotoxicity against $\mathrm{K} 562$ cells with an $\mathrm{IC}_{50}$ value of $0.38 \mu \mathrm{M}$, while compounds 19 and eugenials E (20) exhibited inhibitory activity towards K562 cells with $\mathrm{IC}_{50}$ values of 1.9 and $4.97 \mu \mathrm{M}$, respectively. Compounds 18-20 inhibited the growth of B16F10 cells with $\mathrm{IC}_{50}$ values of $6.0,3.2$ and $8.8 \mu \mathrm{M}$, respectively [37]. Eugenials C-E (18-20) showed lower inhibitory potential against Nalm-6 cells (Table 1). On the other hand, compound $16\left(\mathrm{IC}_{50}=30.5 \mu \mathrm{M}\right)$ has been reported to be more potent than $17\left(\mathrm{IC}_{50}=42.8 \mu \mathrm{M}\right)$ against K562 cells (Table 1 ). 


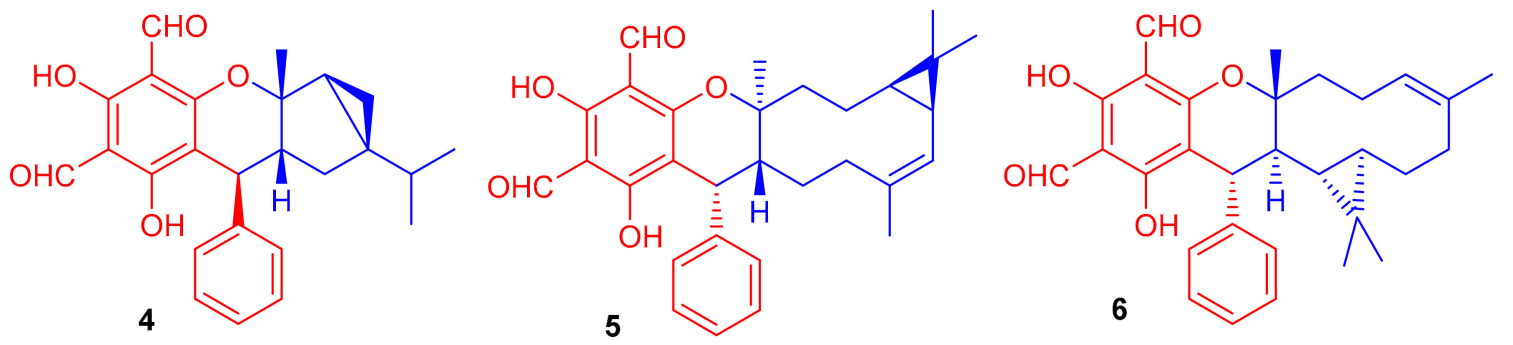

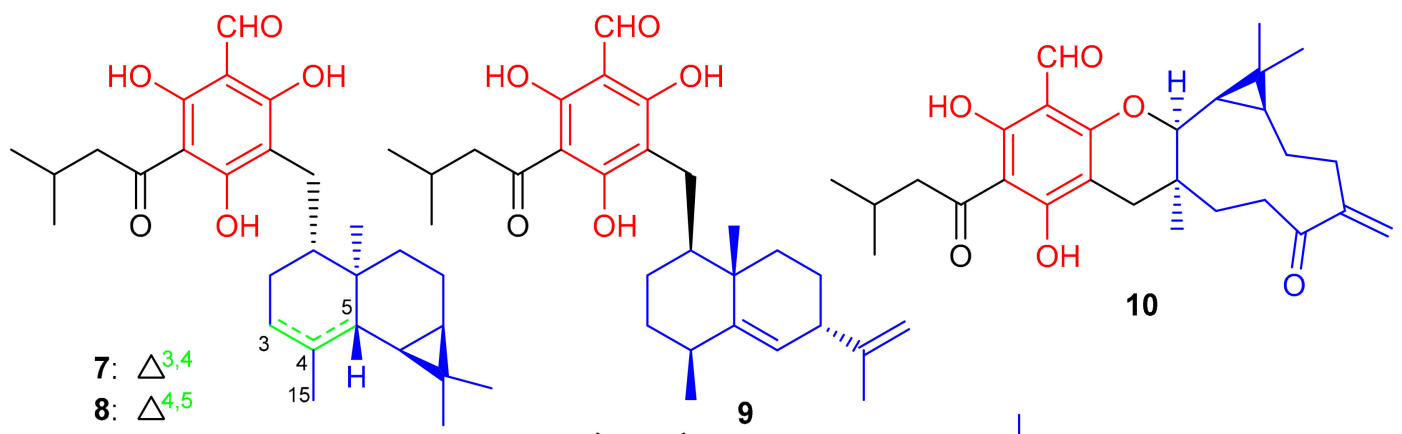

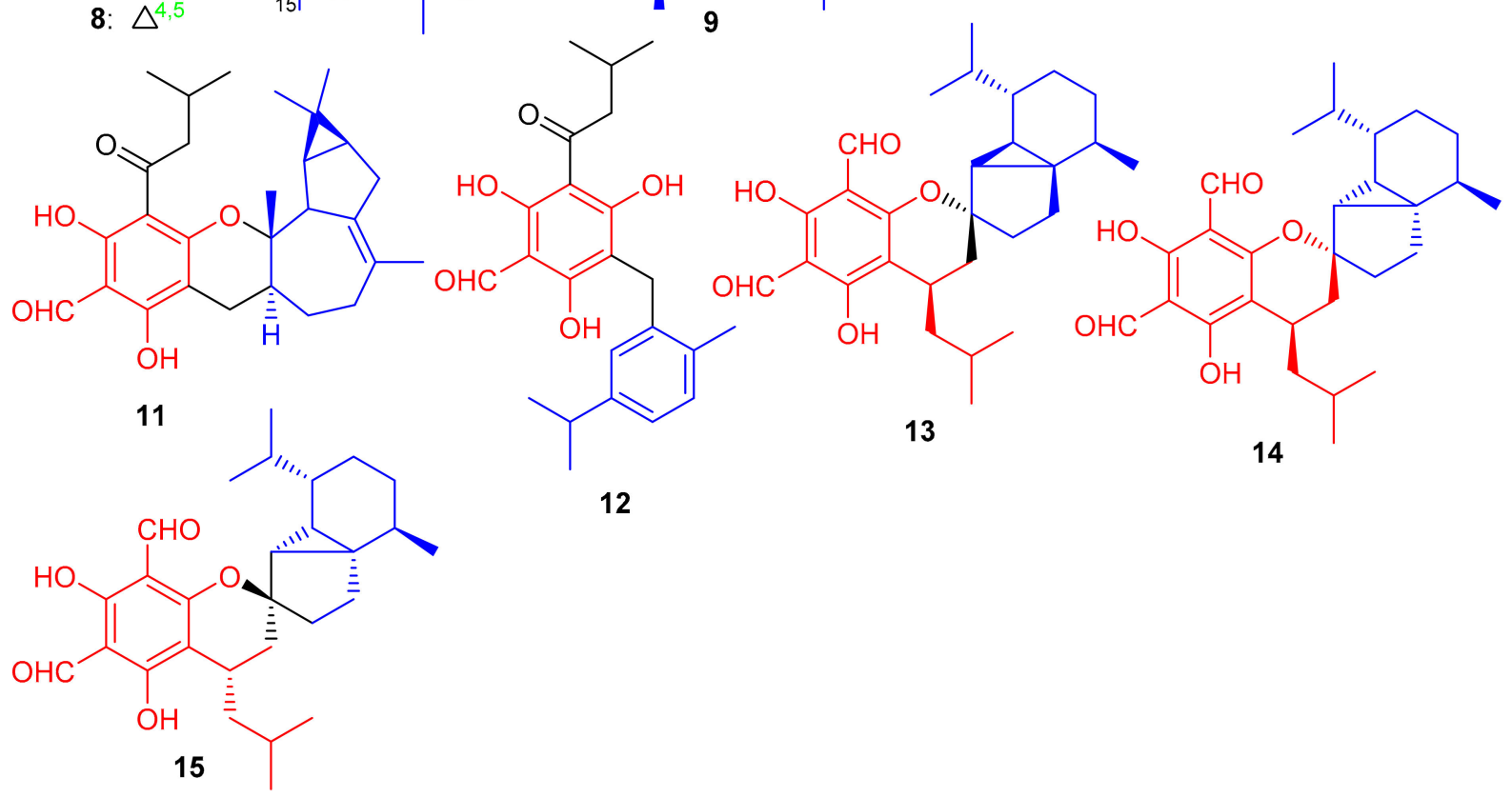

Figure 2. Structures of phloroglucinol-based meroterpenoids 4-15. 


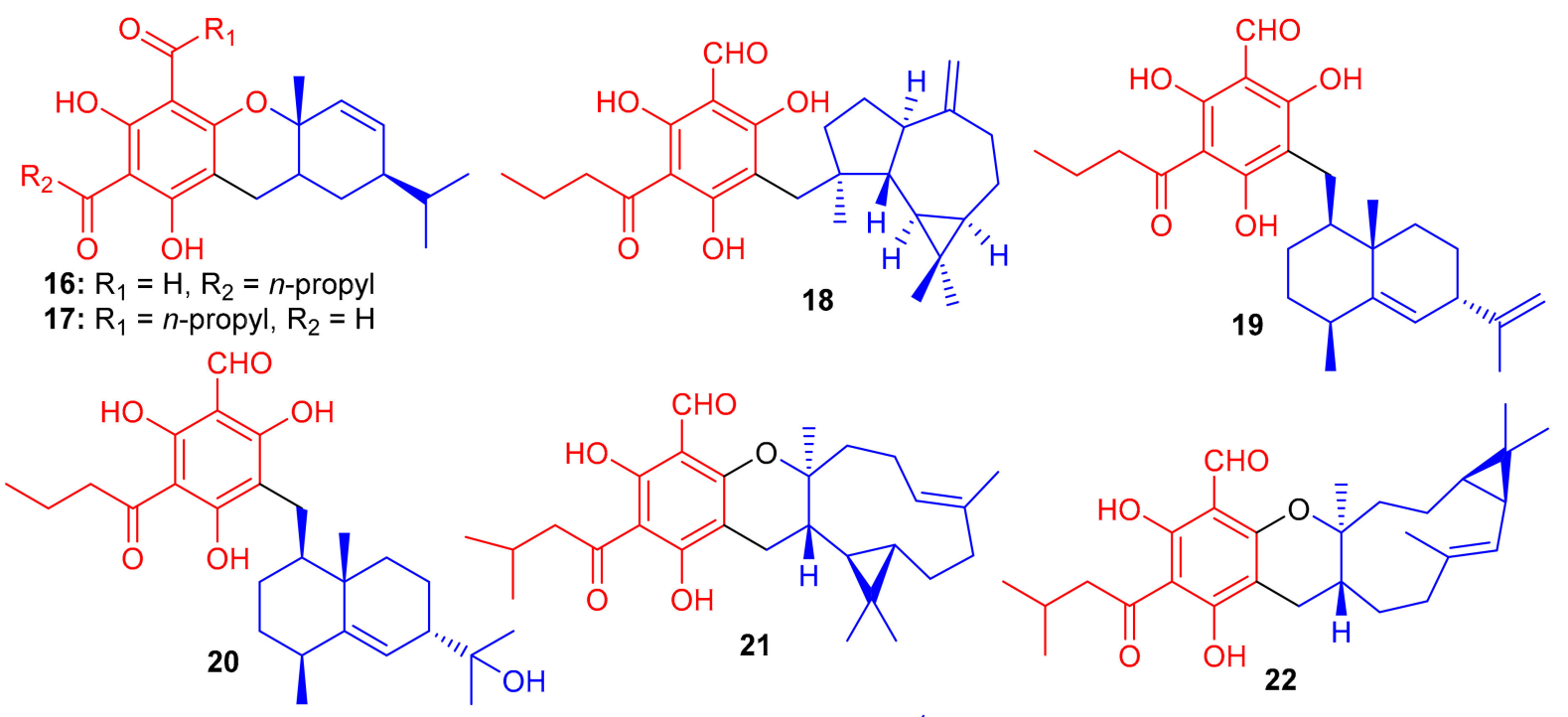

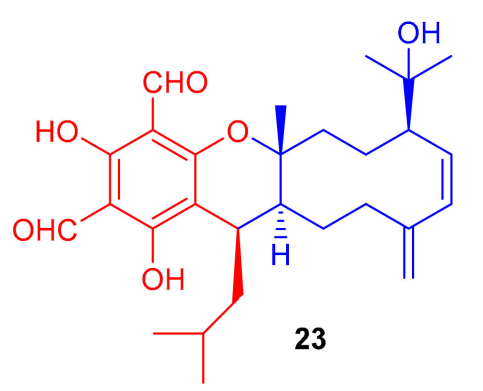

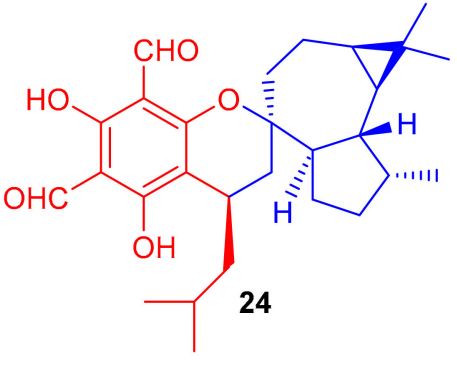

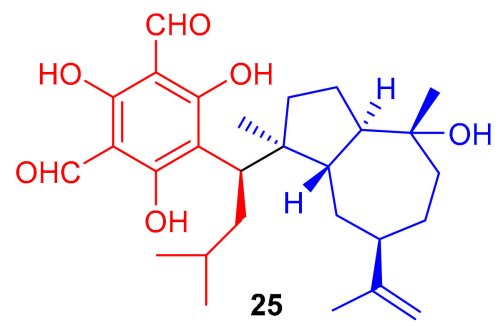

Figure 3. Structures of phloroglucinol-based meroterpenoids 16-25.

Compounds 16 and 17 have fused phloroglucinol-monoterpene systems, while compounds 18-20 do not have similar fused systems but only phloroglucinol-sesquiterpene attachments. Therefore, higher activity of compounds 18-20 can be attributed to the presence of the additional free phenol groups in their structures. Amongst eugenials $\mathrm{C}-\mathrm{E}$ (18-20), the other interesting factor affecting the activity level is their sesquiterpenyl derivatives. Compound $18\left(\mathrm{IC}_{50}=0.38 \mu \mathrm{M}\right)$, which has a tricyclic aromadendrene core, enhances the activity of this compound by 5-fold and 13-fold respectively, which is encouragingly higher than compound $19\left(\mathrm{IC}_{50}=1.9 \mu \mathrm{M}\right)$ and compound $20\left(\mathrm{IC}_{50}=4.97 \mu \mathrm{M}\right)$ [37].

Eucalyptus tereticornis is reported to produce formyl phloroglucinol meroterpenoids, since Liu et al. [38] isolated five formyl phloroglucinol-derived meroterpenoids viz., eucalteretials A-E (21-25). Besides spectroscopic analysis, ECD calculations were used to define the chirality of these compounds. At a concentration of $50 \mu \mathrm{M}$, compound 25 exhibited comparable topoisomerase I (Top1) inhibitory activity to that of camptothecin, whereas, only compound 23 displayed growth inhibition of HCT116 cell lines with an $\mathrm{IC}_{50}$ value of $4.8 \mu \mathrm{M}$ (Table 1). Among compounds 21-25, both 21 and 22 are rare natural products in which the germacrene core unit and the phloroglucinol core are connected in a different pattern (Figure 3) [38].

Eucalyptus globulus fruits are also rich in phloroglucinol-derived meroterpenoids, since several have been isolated previously. Indeed, Qin et al. [39] isolated 10 compounds from this source. The spectroscopic data and ECD calculations lead to their absolute structure determination as eucalypglobulusals A-J (26-35) (Figure 4) [39]. Eucalypglobulusal A (26) has an unusual structure bearing a phloroglucinol core coupled to a rearranged sesquiterpene skeleton. Among these compounds, eucalypglobulusal F (31) inhibited the growth of the human acute lymphoblastic cell line (CCRF-CEM) with an $\mathrm{IC}_{50}$ value of $3.3 \mu \mathrm{M}$ (Table 1 ), which is comparable with the positive control VP-16 $\left(\mathrm{IC}_{50}=1.1 \mu \mathrm{M}\right)$. However, the same compound was not active $\left(\mathrm{IC}_{50}:>10 \mu \mathrm{M}\right)$ towards HCT116, DU145, Huh7 and A549. Moreover, eucalypglobulusal A (26) inhibited DNA topoisomerase I (Top1) [39]. 


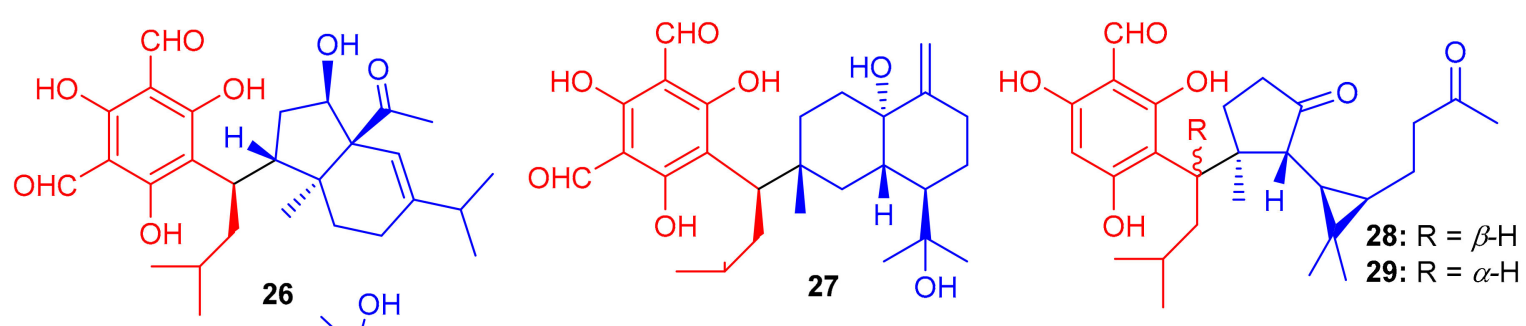

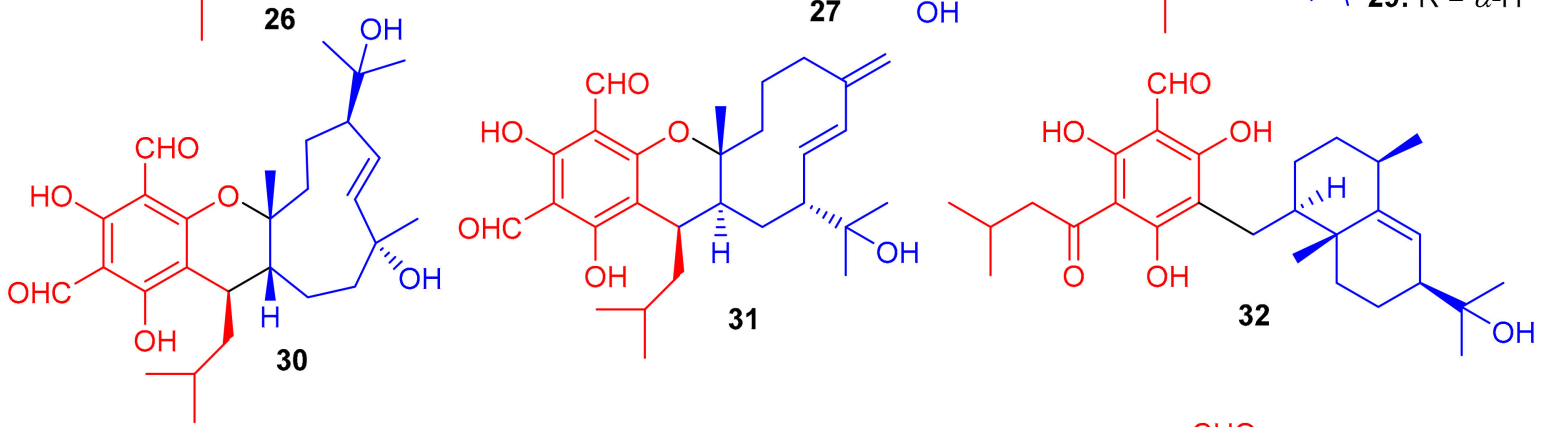<smiles>C=C1CC[C@@H]2[C@@H]([C@@H]3CC[C@](C)(Cc4c(O)c(C=O)c(O)c(C(=O)CC(C)C)c4O)[C@H]13)C2(C)C</smiles><smiles>CC(C)C[C@H]1c2c(O)c(C=O)c(O)c(C=O)c2O[C@]2(C)[C@H](O)[C@H]3O[C@]3(C(C)C)C[C@H]12</smiles><smiles>CC(C)[C@]1(O)C=C[C@]2(C)Oc3c(C=O)c(O)c(C=O)c(O)c3C[C@H]2C1</smiles>

Figure 4. Structures of phloroglucinol-based meroterpenoids 26-35.

Phloroglucinol-based meroterpenoids in the form of enantiomers viz., $( \pm)$-japonicols A-D (36a,b-39a,b) (Figure 5) were isolated from Hypericum japonicum. Moreover only compound 37a illustrated good KSHV activity $\left(\mathrm{EC}_{50}: 8.75 \mu \mathrm{M}\right.$; SI: 16.0$)$ while the other compounds exhibited $\mathrm{EC}_{50}$ values of between 17.6 and $\left.202.9 \mu \mathrm{M}\right)$. Biosynthetically, it is suggested that these acylphloroglucinol-derived meroterpenoids are formed by non-enzymatic reactions, since all were isolated from $H$. japonicum in both enantiomeric forms [40].<smiles>C#CC#CC(C)(C)C(C)(C)O[C@H]1CC[C@H]2Oc3c(c(O)cc(O)c3C(=O)C(C)C)C[C@H]2O1</smiles>

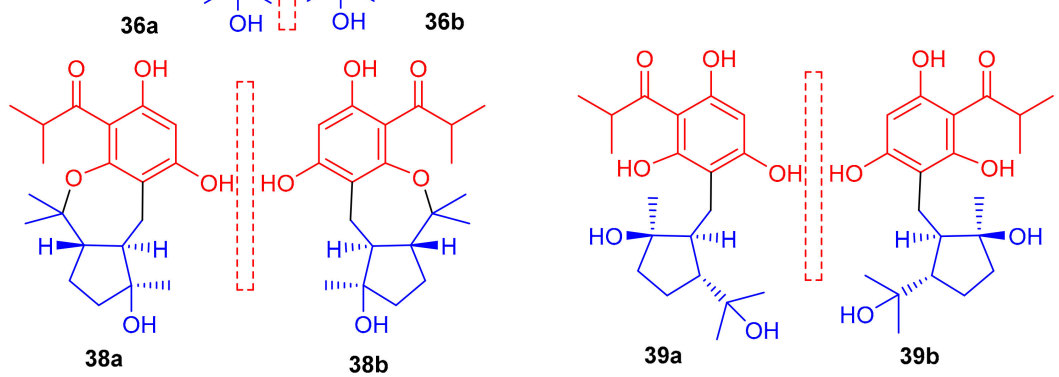

Figure 5. Structures of phloroglucinol-based meroterpenoids 36-39.

New acylphloroglucinol-based meroterpenoid enantiomers viz., (+)-japonicols E$\mathrm{H}(\mathbf{4 0 a} / \mathbf{b}-\mathbf{4 3 a} / \mathbf{b}$ (Figure 6) have been reported from $H$. japonicum and their structures were established via extensive NMR and ECD techniques. Among these compounds, meroterpenes $40 \mathrm{a}$ and $40 \mathrm{~b}$ bore the rare ring unit, cyclopenta[ $[b]$ chromene. Compounds 40a (IC $\mathrm{IC}_{50}: 8.30 \mu \mathrm{M}$; SI: 23.4) and 43a ( $\mathrm{IC}_{50}: 4.90 \mu \mathrm{M}$; SI: 25.7) exhibited inhibitory effects on KSHV (Table 1) [41]. On the other hand, the enantiomers of compounds 40a and 43a 
viz., $40 \mathrm{~b}\left(\mathrm{IC}_{50}: 24.4 \mu \mathrm{M}\right.$ and $43 \mathrm{~b}\left(\mathrm{IC}_{50}: 29.4 \mu \mathrm{M}\right)$ were not especially that active towards KSHV. In addition compound $42 \mathrm{~b}$ illustrated inhibitory effects with $\mathrm{IC}_{50}: 6.7 \mu \mathrm{M}$ and SI: 7.4 while the enantiomer, viz., compound $42 \mathrm{a}$ was not active $\left(\mathrm{IC}_{50}: 21.3 \mu \mathrm{M}\right)$. The authors believe this is illustrative that stereochemistry plays a definite role in enhancing KSHV inhibition. Notably, the activity of $42 \mathbf{a}$ is increased and the selectivity of $43 \mathbf{a}$ is due to the unique phenyl group at C-7' [41].<smiles>C#CC#C[C@H]1Cc2c(O)cc([18OH])c(C(=O)C(C)C)c2O[C@@]1(C)CCC=C(C)C</smiles>

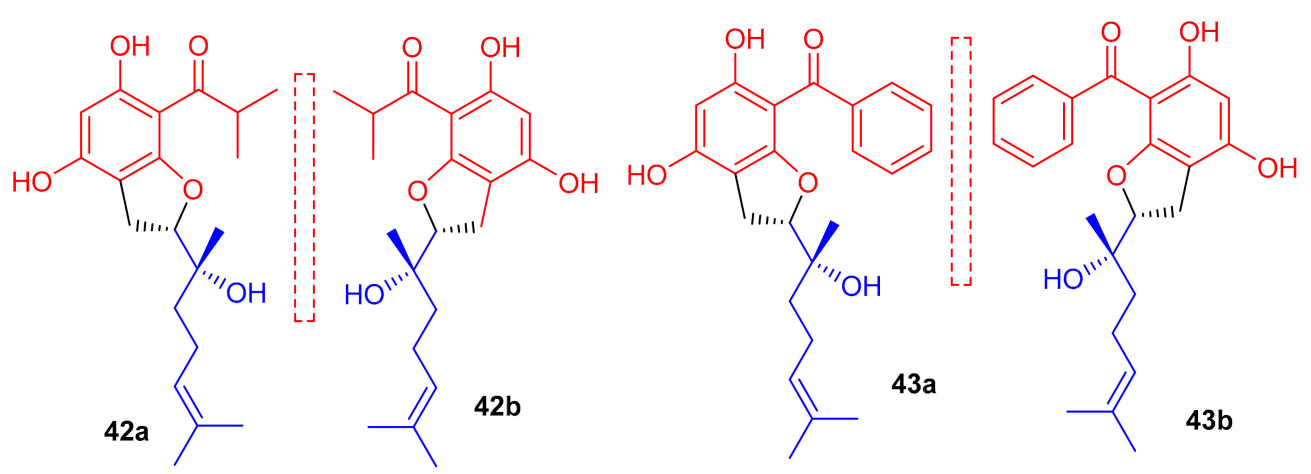

Figure 6. Structures of phloroglucinol-based meroterpenoids 40-43.

Phosphodiesterase-4 (PDE4D-4) inhibitors; psiguajadials A-K (44-54) (Figure 7) were isolated from P. guajava. All these natural products have been reported to be significant inhibitors of PDE4D-4 with $\mathrm{IC}_{50}$ values in the range of 1.34-7.26 $\mu \mathrm{M}$ (Table 1) [42]. This activity potential is comparable with the positive control rolipram, a standard PDE4 inhibitor $\left(\mathrm{IC}_{50} 0.62 \mu \mathrm{M}\right)$. Since a small difference has been reported in the activity level of all these compounds, it may lead to the conclusion that the diformylphloroglucinol moiety is required for PDE4D2 inhibitory activity. The genus Psidium produced a diverse range of meroterpenoids bearing phloroglucinol-coupled to sesquiterpenoids or monoterpenes. As illustrative of this variety, consider phloroglucinol-coupled to the cubebane sesquiterpenoid core to produce compounds 44 and 45 , and compound 46 has globulane as the terpene unit while 49 has caryolane and 50 has caryophyllane, whereas compounds 51-53 have cadinane as the terpene unit [42]. 
<smiles></smiles>

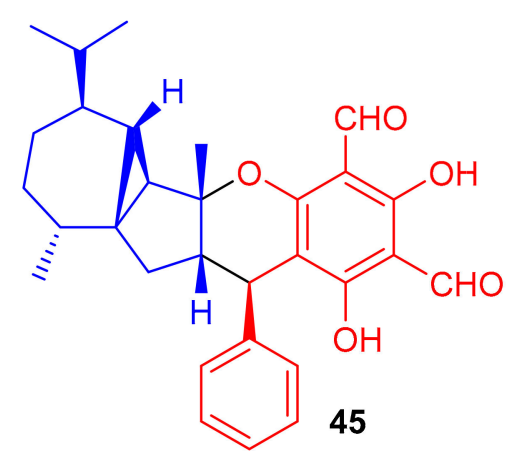

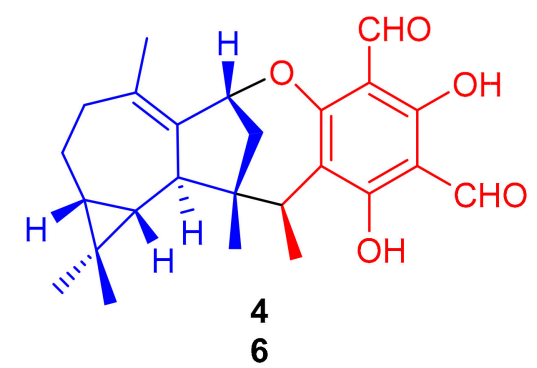<smiles>[Z17]C[C@]1(C)Cc2c(O)c(C=O)c(O)c(C(=O)O)c2O[C@]1(C)CC[C@H]1[C@@H](C)C[C@H]1C</smiles>

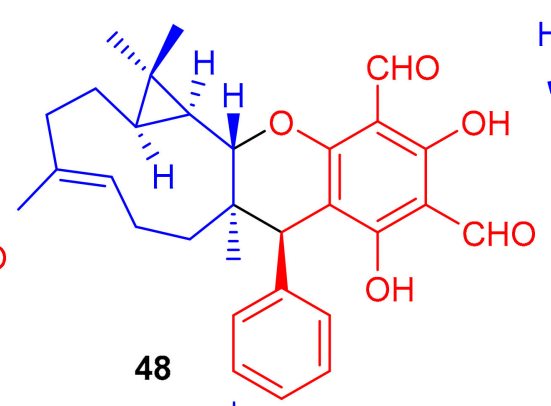

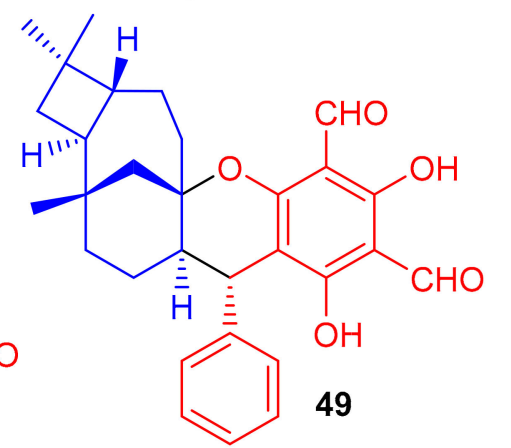<smiles>C=C1CC[C@]2(C)[C@@H](c3ccccc3)c3c(O)c(C=O)c(O)c(C=O)c3O[C@]2(C)CC[C@]12CC2(C)C</smiles><smiles>C/C=C/[C@H](C)C1CC[C@H](C)[C@H]2CC[C@]3(C=C12)C[C@H](c1ccccc1)c1c(O)c(C=O)c(O)c(C=O)c1O3</smiles><smiles>CCCCCCCC[C@@H](C)C(C)C</smiles>

52<smiles>CC(C)[C@H]1CC[C@@H](C)[C@H]2C[C@@H]3c4c(O)c(C=O)c(O)c(C=O)c4O[C@]3(C)C=C12</smiles><smiles>C/C=C\c1c(O)c(C=O)c(O)c2c1O[C@]1(CC[C@@H](C(C)C)CC1)C[C@H]2c1ccccc1</smiles>

54

Figure 7. Structures of phloroglucinol-based meroterpenoids 44-54.

Phytomeroterpenoid eucalrobusones J-P (55-61) and compound 62 (Figure 8) were isolated from Eucalyptus robusta and evaluated for their antifungal activity against $C$. albicans and C. glabrata [43]. Structural diversity among the meroterpenoids 55-62 is generated through the wide range of coupling patterns between the sesquiterpenoid and phloroglucinol units. Meroterpene 55 bearing an unusual carbon skeleton, viz., the 1-oxaspiro[5.6]dodecane unit is formed via the aromadendrane core C-14 rather than C4. On the other hand, metabolite $\mathbf{5 6}$ is a guaiane based meroterpene and interestingly, this compound has two oxo bridges between C-10/C-11 and C-3' /C- 6 which generates the most unusual polycyclic ring system [43]. Moreover, meroterpenes 57-59 are rare aristolane-based meroterpenoids with only a few examples being reported to date [44]. These metabolites showed different microbial inhibitory potentials (Table 1). Compounds 
60 and 62 were moderately active against C. albicans with MIC values of 12.54 and 12.50 $\mu \mathrm{g} / \mathrm{mL}$ respectively, while other members exhibit MIC values of more than $50 \mu \mathrm{g} / \mathrm{mL}$. Compounds 55, 60 and 62 potentially inhibited the growth of C. glabrata with MIC values determined as 2.57, 1.95, and $2.49 \mu \mathrm{g} / \mathrm{mL}$, respectively (Table 1) [43] and these activates are closer to the standard drugs fluconazole (MIC $=0.25 \mu \mathrm{g} / \mathrm{mL})$ and amphotericin B $(\mathrm{MIC}=0.26 \mu \mathrm{g} / \mathrm{mL})$.

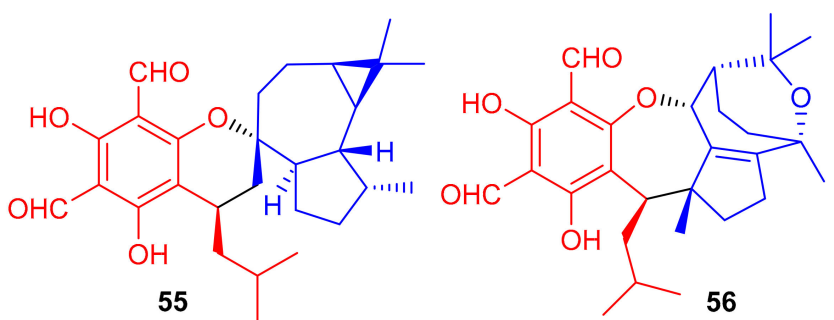

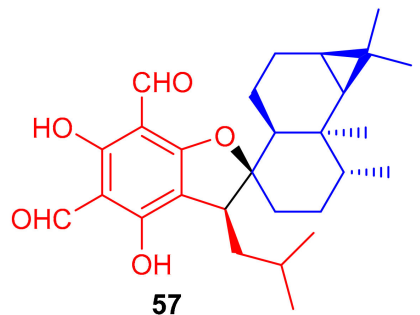

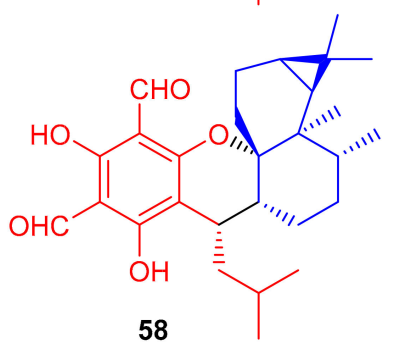<smiles>CC(C)C[C@H](c1c(O)c(C=O)c(O)c(C=O)c1O)C1CC[C@@H](C)[C@]2(C)C1C1C2C1(C)C</smiles>

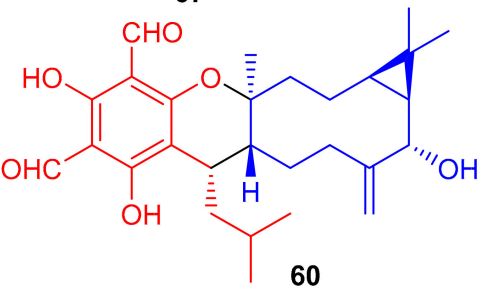<smiles>C=C1CC[C@@]2(C)Oc3c(C=O)c(O)c(C=O)c(O)c3[C@H](CC(C)C)[C@@]2(C)CCC2C(C)C2C(C(=O)C=O)C1(C)C</smiles>

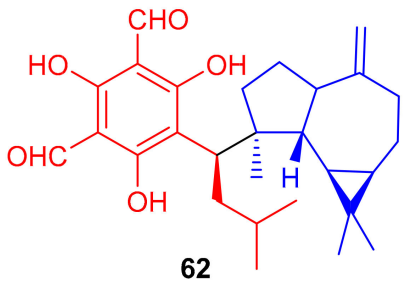

Figure 8. Structures of phloroglucinol-based meroterpenoids 55-62. 
Table 1. Phloroglucinol-Derived Metroterpenoids and their biological effects.

\begin{tabular}{|c|c|c|c|}
\hline Compounds & Source & Activities & Ref. \\
\hline Psiguajavadial A (1) & Psidium guajava & $\begin{array}{l}\text { Cytotoxic effects: HCT116 = } \mathrm{IC}_{50} 7.60 \mu \mathrm{M} ; \\
\text { CCRF-CEM = } \mathrm{IC}_{50} 25.2 \mu \mathrm{M} ; \mathrm{DU} 145=\mathrm{IC}_{50} 20.2 \\
\mu \mathrm{M} ; \mathrm{Huh} 7=\mathrm{IC}_{50} 48.8 \mu \mathrm{M} ; \mathrm{A} 549=\mathrm{IC}_{50} 2.99 \mu \mathrm{M}\end{array}$ & {$[33,34]$} \\
\hline Psiguajavadial B (2) & Psidium guajava & $\begin{array}{l}\text { Cytotoxic effects: HCT116 = } \mathrm{IC}_{50} 21.6 \mu \mathrm{M} ; \\
\text { CCRF-CEM = } \mathrm{IC}_{50} 9.63 \mu \mathrm{M} ; \mathrm{DU} 145=\mathrm{IC}_{50} 26.3 \\
\mu \mathrm{M} ; \mathrm{Huh} 7=\mathrm{IC}_{50} 13.7 \mu \mathrm{M} ; \mathrm{A} 549=\mathrm{IC}_{50} 0.90 \mu \mathrm{M}\end{array}$ & {$[33,34]$} \\
\hline Guadial A (3) & Psidium guajava & $\begin{array}{l}\text { Cytotoxic effects: HCT116 = } \mathrm{IC}_{50} 5.74 \mu \mathrm{M} ; \\
\text { CCRF-CEM = IC } \mathrm{IC}_{50} 2.95 \mu \mathrm{M} ; \mathrm{DU} 145=\mathrm{IC}_{50} 5.35 \\
\mu \mathrm{M} ; \text { Huh7 = IC } \mathrm{IC}_{50} 28.0 \mu \mathrm{M} ; \mathrm{A} 549=\mathrm{IC}_{50} 9.62 \mu \mathrm{M} ; \\
\text { Enzyme Inhibition: PDE4D-4 = } \mathrm{IC}_{50} 2.70 \mu \mathrm{M}\end{array}$ & {$[33,34]$} \\
\hline Guajavadial A (4) & Psidium guajava & $\begin{array}{c}\text { Cytotoxic effects: HL- } 60=\mathrm{IC}_{50} 4.73 \mu \mathrm{M} \text {; A-549= } \\
\mathrm{IC}_{50} 5.62 \mu \mathrm{M} \text {; SMMC-7721 = IC } \mathrm{IC}_{50} 4.37 \mu \mathrm{M} \text {; MCF-7 } \\
=\mathrm{IC}_{50} 22.28 \mu \mathrm{M} ; \mathrm{SW} 480=\mathrm{IC}_{50} 14.55 \mu \mathrm{M} \text {; Enzyme } \\
\text { Inhibition: PDE4D-4 = IC } \mathrm{IC}_{50} 2.01 \mu \mathrm{M}\end{array}$ & [35] \\
\hline Guajavadial B (5) & Psidium guajava & $\begin{array}{l}\text { Cytotoxic effects: HL- } 60=\mathrm{IC}_{50} 6.49 \mu \mathrm{M} ; \mathrm{A}-549= \\
\mathrm{IC}_{50} 5.78 \mu \mathrm{M} ; \mathrm{SMMC}-7721=\mathrm{IC}_{50} 5.05 \mu \mathrm{M} ; \mathrm{MCF}-7 \\
=\mathrm{IC}_{50} 18.02 \mu \mathrm{M} ; \mathrm{SW} 480=\mathrm{IC}_{50} 13.07 \mu \mathrm{M}\end{array}$ & [35] \\
\hline Guajavadial C (6) & Psidium guajava & $\begin{array}{l}\text { Cytotoxic effects: HL-60 = IC } \mathrm{IC}_{50} 3.38 \mu \mathrm{M} ; \mathrm{A}-549= \\
\mathrm{IC}_{50} 5.66 \mu \mathrm{M} \text {; SMMC-7721 = } \mathrm{IC}_{50} 3.54 \mu \mathrm{M} ; \mathrm{MCF}-7 \\
\quad=\mathrm{IC}_{50} 14.54 \mu \mathrm{M} ; \mathrm{SW} 480=\mathrm{IC}_{50} 18.97 \mu \mathrm{M}\end{array}$ & [35] \\
\hline Eucalrobusone A (7) & Eucalyptus robusta & $\begin{array}{c}\text { Cytotoxic effects: HepG2 }=\mathrm{IC}_{50} 18.52 \mu \mathrm{M} ; \text { U2OS } \\
=\mathrm{IC}_{50} 45.00 \mu \mathrm{M}\end{array}$ & [36] \\
\hline Eucalrobusone C (9) & Eucalyptus robusta & $\begin{array}{c}\text { Cytotoxic effects: HepG2 }=\mathrm{IC}_{50} 7.40 \mu \mathrm{M} ; \mathrm{U} 2 \mathrm{OS} \\
=\mathrm{IC}_{50} 8.99 \mu \mathrm{M} ; \mathrm{MCF}-7=\mathrm{IC}_{50} 8.50 \mu \mathrm{M}\end{array}$ & [36] \\
\hline Eucalrobusone D (10) & Eucalyptus robusta & Cytotoxic effects: HepG2 $=\mathrm{IC}_{50} 26.78 \mu \mathrm{M}$ & [36] \\
\hline Eucalrobusone H (14) & Eucalyptus robusta & Cytotoxic effects: $\mathrm{U} 2 \mathrm{OS}=\mathrm{IC}_{50} 42.25 \mu \mathrm{M}$ & [36] \\
\hline
\end{tabular}


Table 1. Cont.

\begin{tabular}{|c|c|c|c|}
\hline Compounds & Source & Activities & Ref. \\
\hline Eugenial B (17) & Eugenia umbelliflora & $\begin{array}{c}\text { Cytotoxic effects: } \mathrm{K} 562=\mathrm{IC}_{50} 42.8 \mu \mathrm{M} \\
\text { Nalm-6 }=\mathrm{IC}_{50} 70.5 \mu \mathrm{M} ; \mathrm{B} 16 \mathrm{~F} 10=\mathrm{IC}_{50} 12.0 \mu \mathrm{M}\end{array}$ & [37] \\
\hline Eugenial C (18) & Eugenia umbelliflora & $\begin{array}{c}\text { Cytotoxic effects: } \mathrm{K} 562=\mathrm{IC}_{50} 0.38 \mu \mathrm{M} \\
\text { Nalm-6 }=\mathrm{IC}_{50} 10.5 \mu \mathrm{M} ; \mathrm{B} 16 \mathrm{~F} 10=\mathrm{IC}_{50} 6.0 \mu \mathrm{M}\end{array}$ & [37] \\
\hline Eugenial D (19) & Eugenia umbelliflora & $\begin{array}{c}\text { Cytotoxic effects: } \mathrm{K} 562=\mathrm{IC}_{50} 1.90 \mu \mathrm{M} ; \\
\text { Nalm-6 }=\mathrm{IC}_{50} 7.75 \mu \mathrm{M} ; \mathrm{B} 16 \mathrm{~F} 10=\mathrm{IC}_{50} 3.20 \mu \mathrm{M}\end{array}$ & [37] \\
\hline Eugenial E (20) & Eugenia umbelliflora & $\begin{array}{c}\text { Cytotoxic effects: } \mathrm{K} 562=\mathrm{IC}_{50} 4.97 \mu \mathrm{M} \\
\text { Nalm-6 = } \mathrm{IC}_{50} 29.1 \mu \mathrm{M} ; \mathrm{B} 16 \mathrm{~F} 10=\mathrm{IC}_{50} 8.80 \mu \mathrm{M}\end{array}$ & [37] \\
\hline Eucalteretials C (23) & Eugenia tereticornis & Cytotoxic effects: $\mathrm{HCT} 116=\mathrm{IC}_{50} 4.8 \mu \mathrm{M}$ & [37] \\
\hline Eucalypglobulusal F (31) & Eugenia globulus & Cytotoxic effects: CCRF-CEM = $\mathrm{IC}_{50} 3.3 \mu \mathrm{M}$ & [37] \\
\hline (+)-Japonicol B (37a) & Hypericum japonicum & Antiviral effects: $\mathrm{KSHV}=\mathrm{EC}_{50} 8.75 \mu \mathrm{M}$ & [40] \\
\hline (+)-Japonicol E (40a) & Hypericum japonicum & Antiviral effects: $\mathrm{KSHV}=\mathrm{IC}_{50}: 8.30 \mu \mathrm{M}$ & {$[41]$} \\
\hline (-)-Japonicol E (40b) & Hypericum japonicum & Antiviral effects: $\mathrm{KSHV}=\mathrm{IC}_{50}: 24.4 \mu \mathrm{M}$ & {$[41]$} \\
\hline (+)-Japonicol G (42a) & Hypericum japonicum & Antiviral effects: $\mathrm{KSHV}=\mathrm{IC}_{50}: 21.3 \mu \mathrm{M}$ & {$[41]$} \\
\hline (+)-Japonicol H (43a) & Hypericum japonicum & Antiviral effects: $\mathrm{KSHV}=\mathrm{IC}_{50}: 4.90 \mu \mathrm{M}$ & [41] \\
\hline (-)-Japonicol H (43b) & Hypericum japonicum & Antiviral effects: $\mathrm{KSHV}=\mathrm{IC}_{50}: 29.4 \mu \mathrm{M}$ & {$[41]$} \\
\hline Psiguajadial A (44) & Psidium guajava & Enzyme Inhibition: PDE4D-4 = $\mathrm{IC}_{50} 3.11 \mu \mathrm{M}$ & [42] \\
\hline Psiguajadial B (45) & Psidium guajava & Enzyme Inhibition: PDE4D-4 = $\mathrm{IC}_{50} 5.03 \mu \mathrm{M}$ & [42] \\
\hline Psiguajadial C (46) & Psidium guajava & Enzyme Inhibition: PDE4D-4 = $\mathrm{IC}_{50} 4.50 \mu \mathrm{M}$ & [42] \\
\hline Psiguajadial D (47) & Psidium guajava & Enzyme Inhibition: PDE4D-4 = $\mathrm{IC}_{50} 4.14 \mu \mathrm{M}$ & [42] \\
\hline Psiguajadial E (48) & Psidium guajava & Enzyme Inhibition: PDE4D-4 = $\mathrm{IC}_{50} 3.25 \mu \mathrm{M}$ & [42] \\
\hline Psiguajadial F (49) & Psidium guajava & Enzyme Inhibition: PDE4D-4 = $\mathrm{IC}_{50} 2.63 \mu \mathrm{M}$ & [42] \\
\hline Psiguajadial G (50) & Psidium guajava & Enzyme Inhibition: PDE4D-4 = $\mathrm{IC}_{50} 1.34 \mu \mathrm{M}$ & [42] \\
\hline Psiguajadial H (51) & Psidium guajava & Enzyme Inhibition: PDE4D-4 = $\mathrm{IC}_{50} 1.81 \mu \mathrm{M}$ & [42] \\
\hline Psiguajadial I (52) & Psidium guajava & Enzyme Inhibition: PDE4D-4 = $\mathrm{IC}_{50} 2.51 \mu \mathrm{M}$ & [42] \\
\hline
\end{tabular}


Table 1. Cont.

\begin{tabular}{|c|c|c|c|}
\hline Compounds & Source & Activities & Ref. \\
\hline Psiguajadial J (53) & Psidium guajava & Enzyme Inhibition: PDE4D-4 = $\mathrm{IC}_{50} 2.53 \mu \mathrm{M}$ & [42] \\
\hline Psiguajadial K (54) & Psidium guajava & Enzyme Inhibition: PDE4D-4 = $\mathrm{IC}_{50} 3.68 \mu \mathrm{M}$ & {$[42]$} \\
\hline Guapsidial A (56) & Psidium guajava & Enzyme Inhibition: PDE4D-4 = $\mathrm{IC}_{50} 5.61 \mu \mathrm{M}$ & [42] \\
\hline Psiguajadial L (57) & Psidium guajava & Enzyme Inhibition: PDE4D-4 = $\mathrm{IC}_{50} 1.37 \mu \mathrm{M}$ & [42] \\
\hline Eucarobustol A (63) & Eucalyptus robusta & Enzyme Inhibition: $\mathrm{PTP} 1 \mathrm{~B}=\mathrm{IC}_{50} 1.3 \mu \mathrm{M}$ & [44] \\
\hline Eucarobustol B (64) & Eucalyptus robusta & Enzyme Inhibition: $\mathrm{PTP} 1 \mathrm{~B}=\mathrm{IC}_{50} 4.3 \mu \mathrm{M}$ & [44] \\
\hline Eucarobustol C (65) & Eucalyptus robusta & Enzyme Inhibition: PTP1B $=\mathrm{IC}_{50} 4.3 \mu \mathrm{M}$ & [44] \\
\hline Eucarobustol D (66) & Eucalyptus robusta & Enzyme Inhibition: PTP1B $=\mathrm{IC}_{50} 2.9 \mu \mathrm{M}$ & {$[44]$} \\
\hline Eucarobustol E (67) & Eucalyptus robusta & Enzyme Inhibition: $\mathrm{PTP} 1 \mathrm{~B}=\mathrm{IC}_{50} 4.1 \mu \mathrm{M}$ & {$[44]$} \\
\hline Eucarobustol F (68) & Eucalyptus robusta & Enzyme Inhibition: PTP1B $=\mathrm{IC}_{50} 5.6 \mu \mathrm{M}$ & [44] \\
\hline Eucarobustol G (69) & Eucalyptus robusta & Enzyme Inhibition: PTP1B $=\mathrm{IC}_{50} 1.8 \mu \mathrm{M}$ & [44] \\
\hline Eucarobustol H (70) & Eucalyptus robusta & Enzyme Inhibition: PTP1B $=\mathrm{IC}_{50} 3.0 \mu \mathrm{M}$ & [44] \\
\hline Eucalyptusdimers A (73) & Eucalyptus robusta & Enzyme Inhibition: $\mathrm{AChE}=\mathrm{IC}_{50} 17.71 \mu \mathrm{M}$ & {$[44]$} \\
\hline
\end{tabular}


Eucalyptus robusta produces eucarobustols A-I (63-71) (Figure 9), which have been identified as PTP1B inhibitors, since all these isolates displayed significant inhibitory potential $\left(\mathrm{IC}_{50}=1.3-5.6 \mu \mathrm{M}\right.$, Table 1$)$. In this assay, the standard compound oleanolic acid inhibited the enzyme activity with an $\mathrm{IC}_{50}$ value of $2.34 \mu \mathrm{M}$ [44]. The published results (Table 1$)$ revealed that compounds $63\left(\mathrm{IC}_{50}: 1.3 \mu \mathrm{M}\right), 67\left(\mathrm{IC}_{50}: 1.8 \mu \mathrm{M}\right)$, and $85\left(\mathrm{IC}_{50}: 1.6 \mu \mathrm{M}\right)$ were even more potent than oleanolic acid. It is speculated from the cases of the two pairs of epimers (63/64 and 69/70) that the relative configuration of $\mathrm{H}-9^{\prime}$ can play a central role in the activity and that this provides useful information for further investigations into a structure-activity relationship [44]. Metabolite 65 displays an unusual coupling moiety of acylphloroglucinol and guaiane through the C-4-C-7' bond. Compound 63 has an acylphloroglucinol coupled sesquiterpene viz., aristolane-type while compounds 67-71 have aromadendrane type sesquiterpene linked to acylphloroglucinol units. Guavadial (72), isolated from Psidium guajava L. has caryophyllene attached to a diformyl phloroglucinol core [45]. Eucalyptusdimers A-C (73-75) were reported from Eucalyptus robusta and were identified via intensive spectroscopic methods. These compounds were shown to bear a fused skeleton between two acylphloroglucinol and two phellandrene cores. Furthermore only compound 73 illustrated anti-AChE effects with an $\mathrm{IC}_{50}: 17.71 \mu \mathrm{M}$ [46].

Meroterpenoids, callisalignenes A-C (76-78) (Figure 10) were produced by Callistemon salignus and these compounds were not active in antimicrobial screening [47]. Meroterpenoids, drychampones A-C (79-81), were produced by Dryopteris championii. Moreover compounds 80 and 81 featuring an 11/6/6 core coupled with a pyronone moiety [48]. Guajavadimer A (82) was produced by Psidium guajava and illustrated moderate hepatoprotective effects. Moreover guajavadimer A (82) featured unusual dimeric meroterpenoids bearing two caryophyllene skeletons coupled with a benzylphloroglucinol along with flavonone unit [49].

$( \pm)$-Dryocrassoids E-J [( \pm -83-88], (Figure 11) were reported from Dryopteris crassirhizoma and these metabolites illustrated moderate anti-HSV-1, and anti-RSV effects [50]. Psiguadiols A-J (89-98) were produce by Psidium guajava and evaluated for PTP1B inhibition. Moreover, compounds $83\left(\mathrm{IC}_{50}: 4.7 \mu \mathrm{M}\right), 95\left(\mathrm{IC}_{50}: 6.2 \mu \mathrm{M}\right)$, and $96\left(\mathrm{IC}_{50}: 9.2 \mu \mathrm{M}\right)$ demonstrated significant PTP1B inhibition while meroterpenes 90-92 illustrated good inhibition with $\mathrm{IC}_{50}$ of $11.0,11.9$, and $10.7 \mu \mathrm{M}$, respectively. On the other hand the remaining compounds possess PTP1B inhibition with $\mathrm{IC}_{50}$ of $<23.0$ while all compounds were more potent than standard oleanolic acid $\left(\mathrm{IC}_{50}: 40.7 \mu \mathrm{M}\right)$ [51].

Littordials A-E (99-103), (Figure 12) phloroglucinol- $\beta$-caryophyllene coupled meroterpenoids were produce by Psidium littorale. Moreover, meroterpenes 100, 101 and 103 illustrated cytotoxic effects towards $\mathrm{B} 16$ and $\mathrm{MDA}-\mathrm{MB}-231$ with $\mathrm{IC}_{50}$ : ranging from 6.6 to $9.2 \mu \mathrm{M}$ [52]. Meroterpenes, eucalrobusone Q-Z (104-115) were isolated from Eucalyptus robusta and these metabolites have terpene moieties such as cadinane, aromadendrane, cubebane, pinane and aromatic menthane. (+)-Eucalrobusone $X(113)$ illustrated the most significant antifungal effects towards Candida albicans $\left(\mathrm{MIC}_{50}: 10.7 \mu \mathrm{g} / \mathrm{mL}\right.$ ) while eucalrobusone U (109) demonstrated the highest anti-C. glabrata effects with $\mathrm{MIC}_{50}: 1.53 \mu \mathrm{g} / \mathrm{mL}$ [53]. 

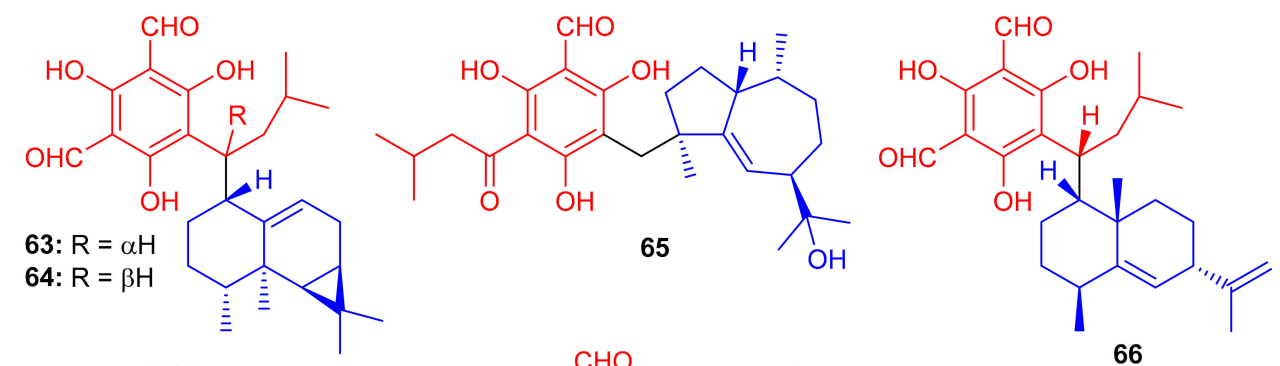

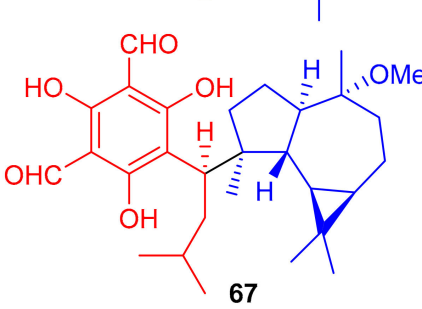<smiles>CC(C)C[C@H](c1c(O)c(C=O)c(O)c(C=O)c1O)[C@H]1CC[C@H]2[C@H](C)CCC3[C@@H]([C@@H]32)C1(C)C</smiles>

68

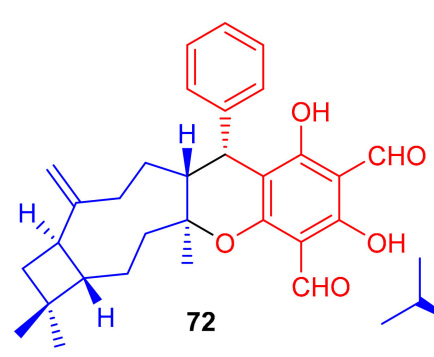<smiles>CC(C)C[C@H](c1c(O)c(C=O)c(O)c(C=O)c1O)[C@H]1CCC2=C1[C@H]1[C@@H](CC[C@H]2C)C1(C)C</smiles>

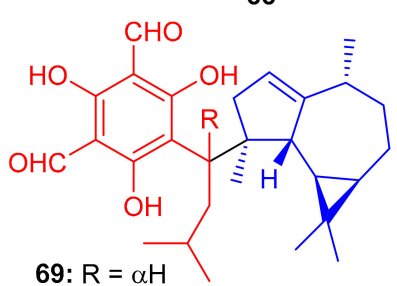

70: $\mathrm{R}=\beta \mathrm{H}$

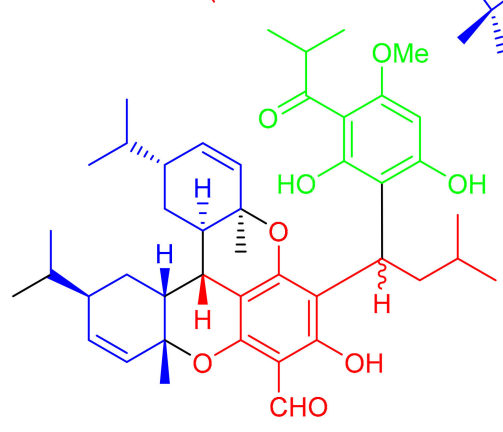<smiles>CC[C@H](C)COC</smiles>

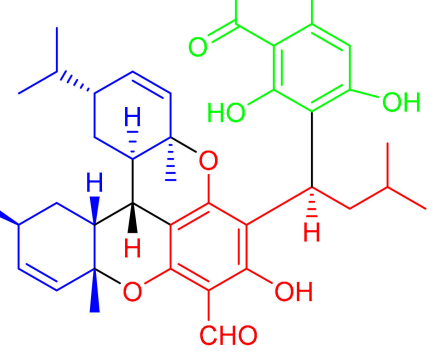

73

\section{4: $9 \mathrm{R}$}

75: $9 S$

Figure 9. Structures of phloroglucinol-based meroterpenoids 63-75.

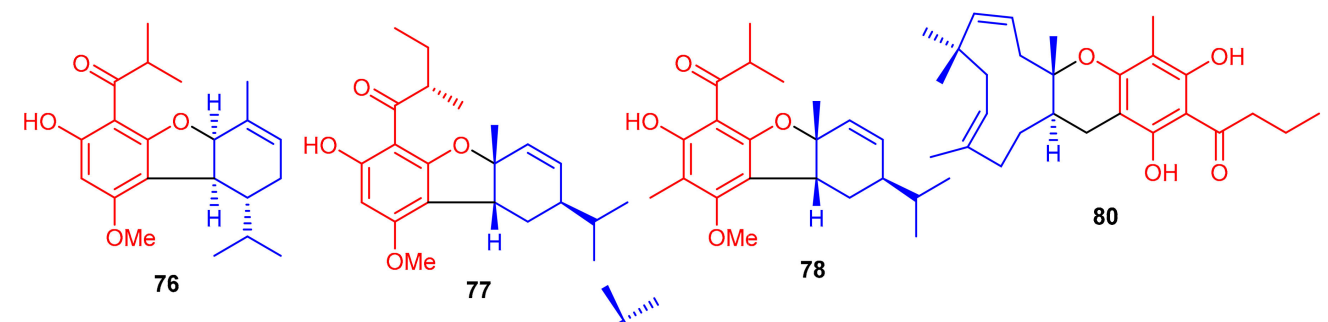

76

$$
77
$$

78<smiles>[R]CC(=O)c1c(O)c(Cc2cc(O)c(C)oc2=O)c2c(c1O)C[C@@H]1CC/C(C)=C\CC(C)(C)/C=C/C[C@@](C)(O)OC[C@@H]21</smiles>

$\begin{aligned} & \text { 79: } R=E t \\ & \text { 81: } R=H\end{aligned}$

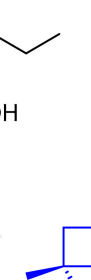<smiles>CC1(C)CCCCC1</smiles> 
<smiles>[R]C(=O)c1c(O)cc2c(c1O)C[C@H](CC/C(C)=C/CCC(C)(O)C=C)C(C)(C)O2</smiles>

(+)-83 10'S R $=n \mathrm{Pr}$

$(-)-8310^{\prime} R \mathrm{R}=n \mathrm{Pr}$

(+)-85 10'S R = Et

$(-)-85 \quad 10^{\prime} R \mathrm{R}=\mathrm{Et}$

$(+)-8710^{\prime} \mathrm{S} R=\mathrm{Me}$

$(-)-87 \quad 10^{\prime} R \mathrm{R}=\mathrm{Me}$<smiles>[R]C(=O)c1c(O)cc2c(c1O)C[C@H](CCC(C)(O)C=C)[C@@](C)(/C=C\C=C(C)C)O2</smiles>

(+)-84 6'S, 7'R R = nPr

(-)-84 6'R, 7'S R $=n \mathrm{Pr}$

$(+)-866^{\prime} S, 7^{\prime} R \quad R=E t$

$(-)-86 \quad 6^{\prime} R, 7^{\prime} S \mathrm{R}=\mathrm{Et}$

$(+)-886^{\prime} S, 7^{\prime} R \mathrm{R}=\mathrm{Me}$

$(-)-88$ 6'R, 7'S R $=\mathrm{Me}$

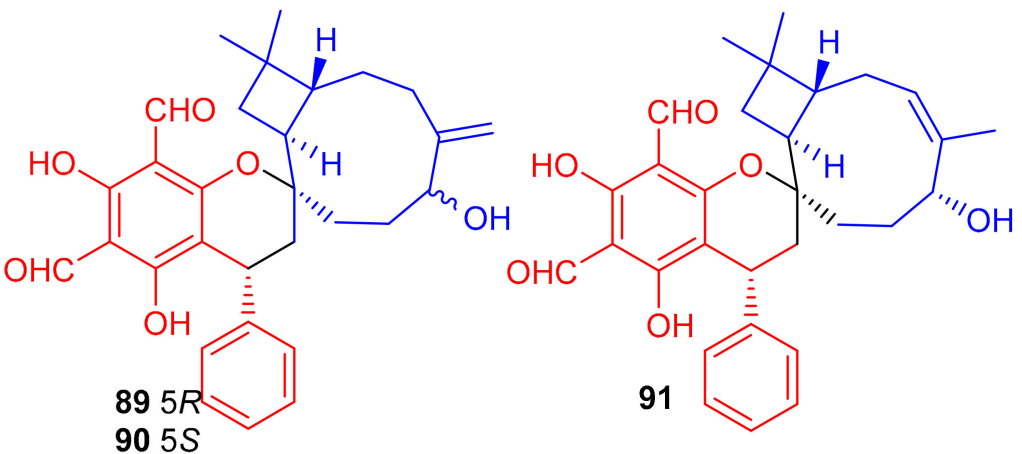

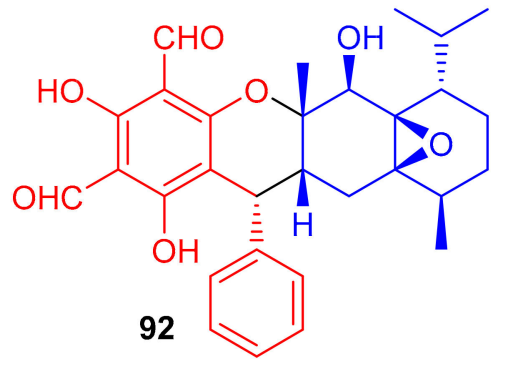

$905 S$

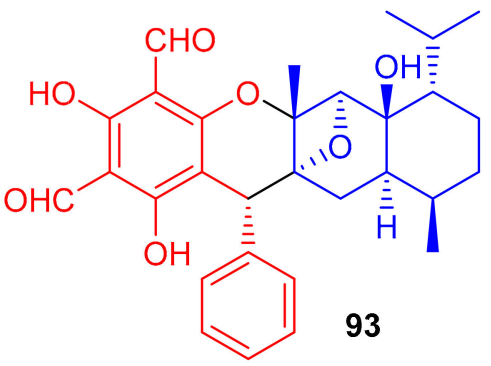

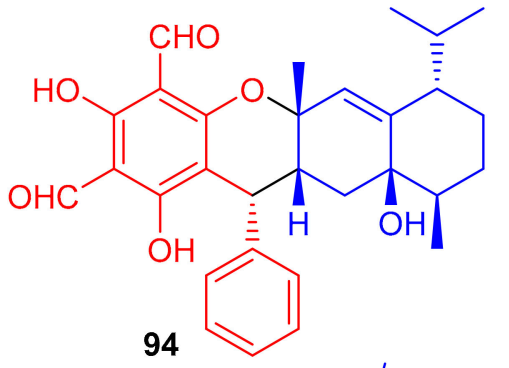<smiles>CC(C)[C@H]1CC[C@H](C)[C@]2(C)C[C@]3(O)[C@H](c4ccccc4)c4c(O)c(C=O)c(O)c(C=O)c4O[C@]3(C)C=C12</smiles>

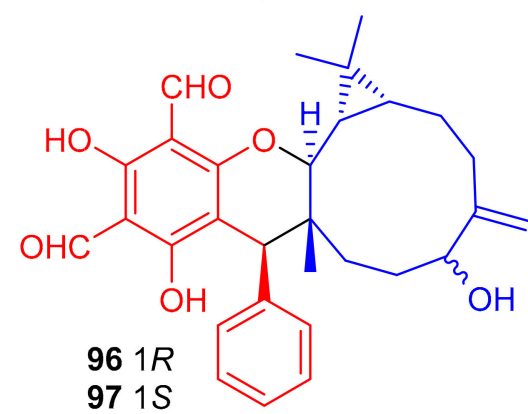<smiles>C/C1=C/C[C@@H]2[C@@H]([C@@H]3Oc4c(C=O)c(O)c(C=O)c(O)c4[C@@H](c4ccccc4)[C@@]3(C)CC[C@@H]1O)C2(C)C</smiles>

Figure 11. Structures of phloroglucinol-based meroterpenoids 83-98. 


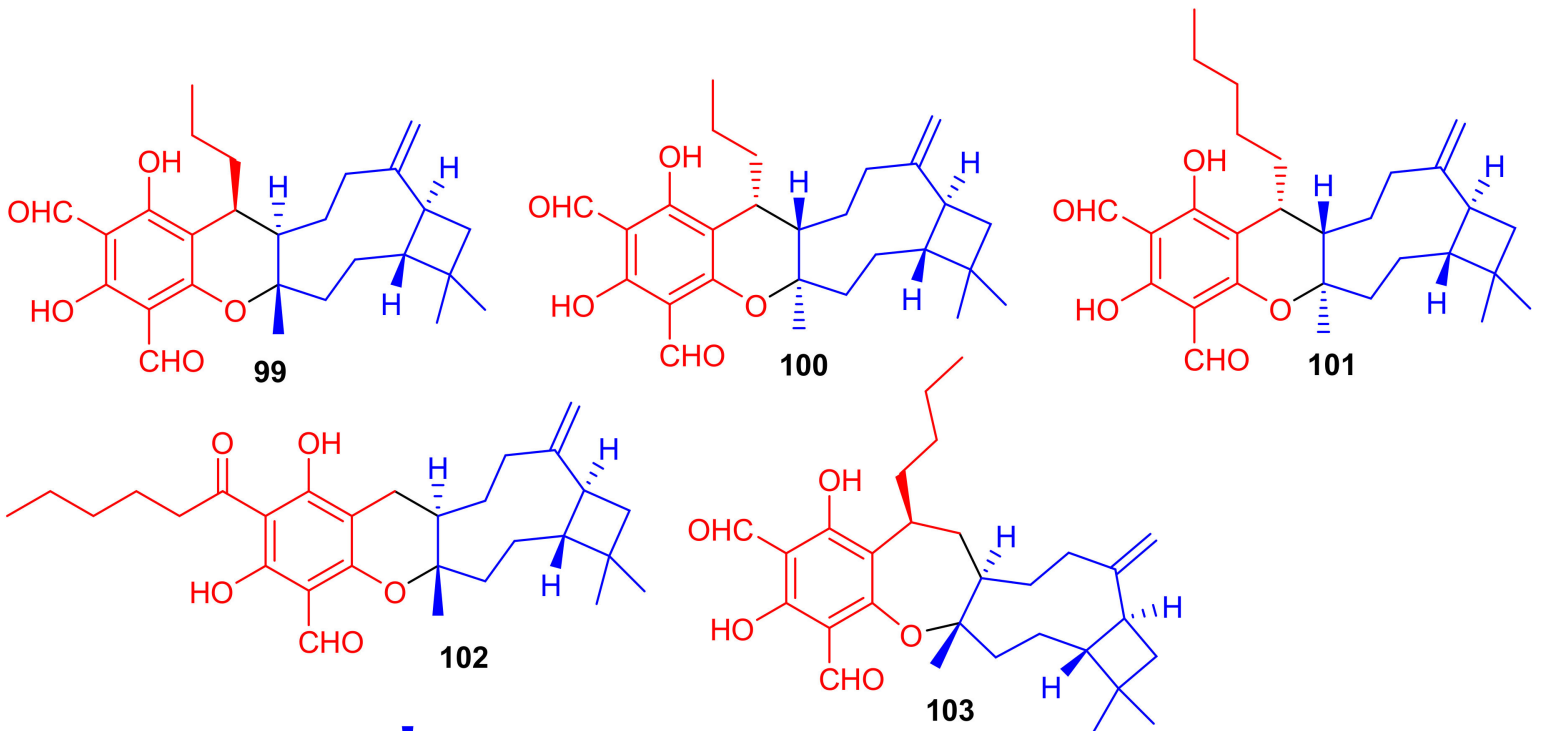<smiles>CC1=C2CC[C@](C)([C@H](CC(C)C)c3c(O)c(C=O)c(O)c(C=O)c3O)[C@H]3OC(C)(C)[C@@H](CC1)[C@@]23C</smiles>

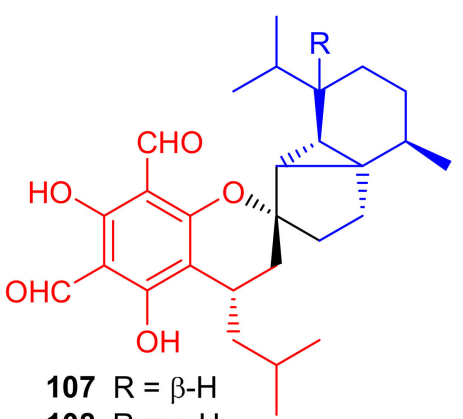

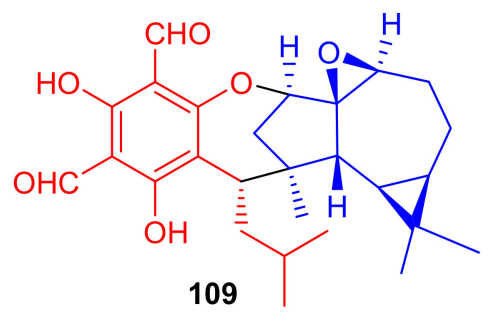

$108 \mathrm{R}=\alpha-\mathrm{H}$<smiles>CC1C[C@H](c2c(O)c(C=O)c(O)c(C=O)c2O)[C@@]2(CC[C@@]3(O)[C@@H](C)CCC4[C@H]3[C@@H]4C2(C)C)C1</smiles><smiles>CCCC12CC1(C)c1c(O)c(C=O)c(O)c(C=O)c1O[C@@](C)(c1ccc(C)cc1)C2</smiles>

( $\pm 112 \mathrm{R}=\beta-\mathrm{H}$

( \pm ) $113 \mathrm{R}=\alpha-\mathrm{H}$

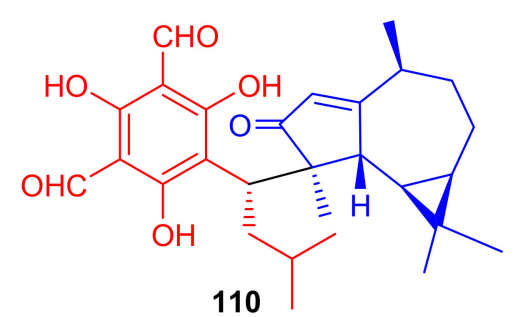<smiles>CC(C)C[C@H](c1c(O)c(C=O)c(O)c(C=O)c1O)[C@@]1(C)CC[C@]23CC1[C@@H](C(C)(C)O)CC[C@H]2C3</smiles>

106<smiles>CC(C)C[C@H]1c2c(O)c(C=O)c(O)c(C=O)c2O[C@H]1C1=CC2CCC1(C)C2(C)C</smiles><smiles>CC(C)C[C@@H](c1c(O)c(C=O)c(O)c(C=O)c1O)C(O)C1=CCC2CC1C2(C)C</smiles>

Figure 12. Structures of phloroglucinol-based meroterpenoids 99-115.

Melaleucadines A (116) and B (117), (Figure 13) phloroglucinol-terpene meroterpenoid metabolites have the phloroglucinol unit coupled with $\beta$-pinene to form the humulene-type sesquiterpenes respectively. Moreover, meroterpenes 116 and 117 illustrated a significant 
neuroprotective effect with cell viability of $53.7 \%$ and $58.3 \%$, respectively [54]. Littordial F (118) was isolated from Psidium littorale which has an unusual 6/8/9/4-tetracyclic core. This compound exhibited moderate cytotoxic effects on B16, MDA-MB-321, and A549 cells with $\mathrm{IC}_{50}$ ranging from 21.5 to $41.5 \mu \mathrm{M}$ [55]. Phloroglucinol based meroterpenes named fischernolides A-D (119-122), having a diterpene core (Figure 13), were produced by Euphorbia fischeriana. Meroterpenes 120 and 122 illustrated significant cytotoxic effects towards HT-29, Bel-7402, MCF-7, A549, and HeLa with $\mathrm{IC}_{50}$ values from 2.0 to $8.6 \mu \mathrm{M}$ [56]. Euphoractone (123) was isolated from Euphorbia fischeriana and displayed cytotoxic effects towards $\mathrm{H} 23$ and $\mathrm{H} 460$ cells with the $\mathrm{IC}_{50}$ : 21.0 and $20.9 \mathrm{mmol} / \mathrm{L}$ respectively [57].<smiles>COc1cc2c(c(O)c1C)[C@@H](c1ccccc1)c1cc(OC)c(C)c(O)c1[C@@H](c1ccccc1)C[C@]1(CO2)CC2CC1C2(C)C</smiles><smiles>C=C1CC[C@H](CC[C@@]2(C)C[C@@H](C)Cc3c(O)c(C=O)c(O)c(C=O)c3O2)[C@@H](C)CC1(C)C</smiles>

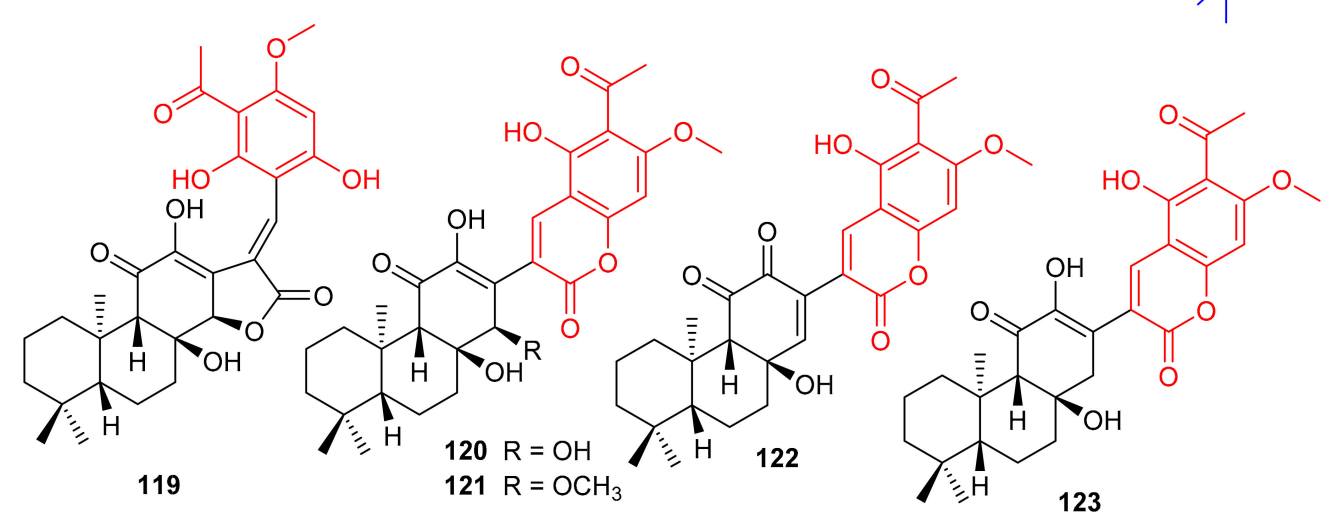

Figure 13. Structures of phloroglucinol-based meroterpenoids 116-123.

\section{Syncarpic Acid/ $\beta$-Triketones-Based Meroterpenes}

Baeckea frutescens has been reported to produce a small library of polymethylated phloroglucinol meroterpenoids, viz., baeckfrutones A-L (124-135) (Figure 14). The structures of these metabolites were also elucidated via spectroscopic methods, X-ray diffraction techniques, and ECD calculations [58]. Meroterpenoids 124 and 125-127 are novel hybrid compounds of the enone-type phloroglucinols linked with phellandrene and sabinene, respectively. Biogenetically, meroterpenoids 124-135 involve regio- and stereoselective [4 +2$]$ cycloaddition reactions between demethylated tasmanone or tasmanone and monoterpenoids, viz., sabinene, $\beta$-phellandrene, and $\beta$-piene. Enone-type phloroglucinol (-)-125 $\left(\mathrm{IC}_{50}: 1.33 \mu \mathrm{M}\right)$ and $133\left(\mathrm{IC}_{50}: 4.04 \mu \mathrm{M}\right)$ showed potent inhibition of DU145 cell lines, which is more potent than VP-16, the standard inhibitor $\left(\mathrm{IC}_{50}=5.22 \mu \mathrm{M}\right)$. On the other hand, compounds 129 and 134 displayed moderate growth inhibition of $\mathrm{A} 549\left(\mathrm{IC}_{50}: 15.6 \mu \mathrm{M}\right)$ and HCT116 $\left(\mathrm{IC}_{50}: 12.8 \mu \mathrm{M}\right)$ cell lines (Table 2). Moreover compounds 129 (74.4\%), 130 (75.3\%), (+)-132 (55.1\%), and $\mathbf{1 3 1}(75 \%)$ illustrated significant anti-inflammatory effects. Additionally, compound $133\left(\mathrm{IC}_{50}: 43.0 \mu \mathrm{M}\right)$ displayed good effects towards AChE. The remainder of the metabolites were either weakly active or inactive [58]. 


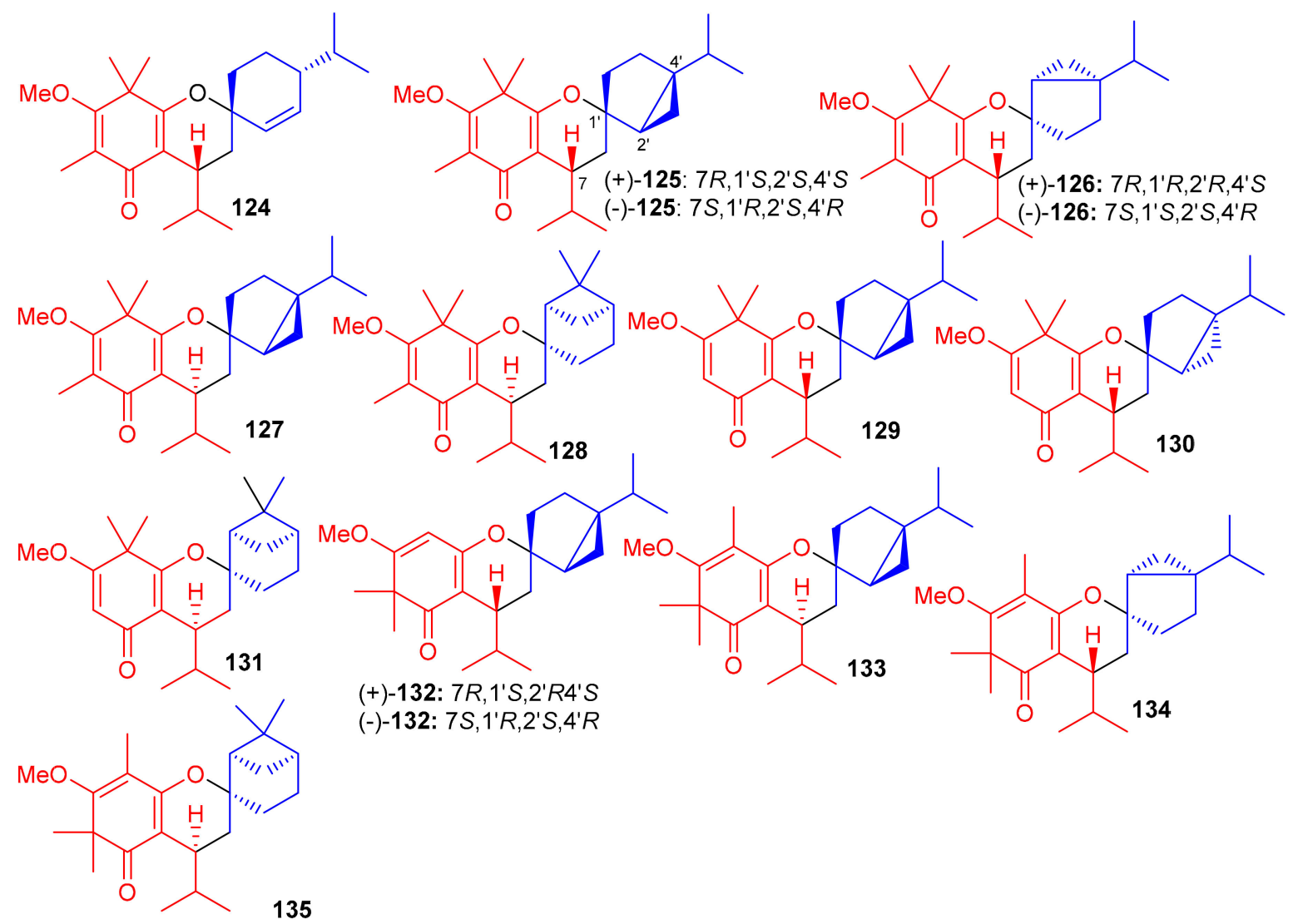

Figure 14. Structures of syncarpic acid/ $\beta$-triketones-based meroterpenes 124-135.

Further phloroglucinol-derived phytomeroterpenoids, named baeckfrutones $\mathrm{M}-\mathrm{S}$ (136-142) (Figure 15), were isolated from B. frutescens. Biogenetically, meroterpenoids 136-142 also involve a [4+2] cycloaddition condensation between demethylated tasmanone or tasmanone and monoterpenoids viz., sabinene, thujene, and caryophyllene [59]. Compounds 136-142 were tested for anti-inflammatory activity, in which it was found that only (+)-136 ( $\left.\mathrm{IC}_{50}: 20.8 \mu \mathrm{M}\right)$ and $\mathbf{1 4 2}\left(\mathrm{IC}_{50}: 36.2 \mu \mathrm{M}\right)$ displayed potent effects and their activities were more potent than the positive control L-NG-monomethyl arginine (L-NMMA, $\left.\mathrm{IC}_{50}=54.0 \mu \mathrm{M}\right)$. Moreover, none of these compounds were active towards HL-60, A-549, MCF-7, SW480, and SMMC-7721 cancer cells [59].

Hyperjaponol H (143) (Figure 16), obtained from Hypericum japonicum, was identified with the help of spectroscopic analyses and a comparison of the Cotton effects of an ECD spectrum. Hyperjaponol H (143) is a hybrid of tasmanone and the monoterpene germacrane. An assay on lytic DNA replication of EBV in B95-8 cells indicated that this compound displayed moderate inhibitory effects with an $\mathrm{EC}_{50}$ value of $25.00 \mu \mathrm{M}$ [60] (Table 2). Spectroscopic identification of the secondary metabolites of Rhodomyrtus tomentosa revealed that tomentosenol A (144), $4 S$-focifolidione (145) and $4 R$-focifolidione (146) contain a unique free syncarpic acid-derived meroterpenoid skeleton. Compound 144 was also confirmed through biomimetic synthesis and was shown to potentially inhibit the growth of $S$. aureus with an MIC value of $4.74 \mu \mathrm{M}$, which has been reported to be comparable with the standard drug vancomycin $(\mathrm{MIC}=1.23 \mu \mathrm{M})$. Since the other compounds 113 and 146 have been reported as being inactive, it seems that the pyran ring is responsible for reducing the antibacterial activity. In addition, tomentosenol A (144) moderately inhibited the growth of MCF-7 $\left(\mathrm{IC}_{50}=8.66 \mu \mathrm{M}\right)$, NCI-H460 $\left(\mathrm{IC}_{50}=8.62 \mu \mathrm{M}\right)$, SF-268 $\left(\mathrm{IC}_{50}=10.01\right.$ $\mu \mathrm{M})$ and HepG-2 $\left(\mathrm{IC}_{50}=9.44 \mu \mathrm{M}\right)($ Table 2$)[61]$. 


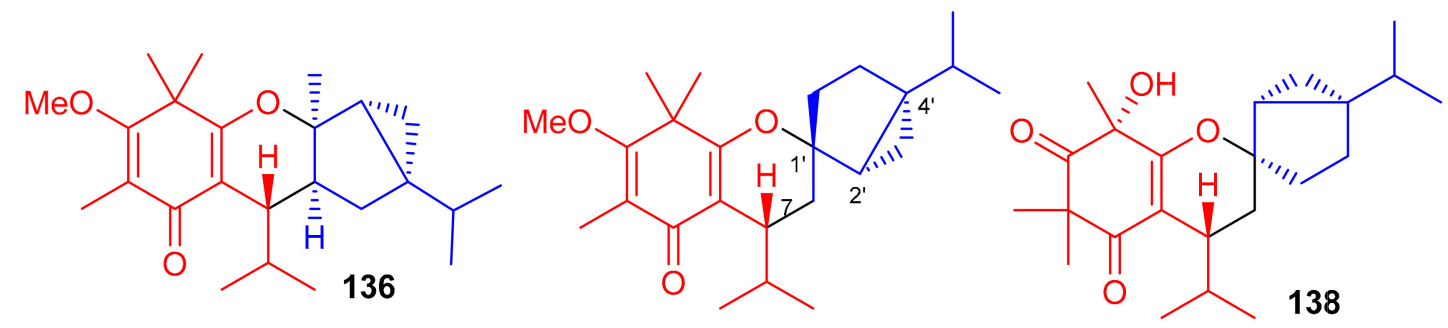

(+)-137: $7 R, 1^{\prime} S, 2^{\prime} S, 4^{\prime} R$

(-)-137: $7 S, 1^{\prime} R, 2^{\prime} R, 4^{\prime} S$<smiles>CC(C)[C@@H]1CC[C@]2(CC3=C(O2)C(C)(O)C(=O)C(C)(C)C3=O)C[C@@H]1C(C)C</smiles><smiles>[R]C1=C(OC)C(C)(C)C(=O)C2=C1O[C@]1(CC[C@H](C(C)C)CC1)C[C@@H](C(C)C)C2=[R4](C)[R4]([H])=C</smiles>

Figure 15. Structures of syncarpic acid/ $\beta$-triketones-based meroterpenes 136-142.<smiles>CC(C)CC[C@H]1CC2=C(OC1=O)C(C)(C)C(=O)C(C(=O)C(C)C)=C2O</smiles><smiles>CC(C)C[C@H](CC1CC2CCC1C2(C)C)C1=C(O)C(C)(C)C(=O)C(C)(C)C1=O</smiles><smiles>CC(C)C[C@H]1C[C@@]2(CC3CCC2C3(C)C)OC2=C1C(=O)C(C)(C)C(=O)C2(C)C</smiles><smiles>CC(C)C[C@H]1C[C@@]2(CC[C@@H]3CC2(C)C3(C)C)OC2=C1C(=O)C(C)(C)C(=O)C2(C)C</smiles>

Figure 16. Structures of syncarpic acid/ $\beta$-triketones-based meroterpenes 143-146.

Liu et al. [62] isolated syncarpic acid-derived meroterpenoids from Myrtus communis. Spectroscopic analysis revealed that myrtucommulones (147-149) (Figure 17) and ( \pm )-150 having a different skeleton to compound 147 affords a unique octahydrospiro\{bicyclo[7.2.0] undecane-2,2'-chromene\} tetracyclic ring system. Compounds 147-149 bear a syncarpic acid coupled with the sesquiterpene viz., caryophyllene while compound $\mathbf{1 5 0}$ has humulene as the sesquiterpene core [54]. In an MTT assay, compound 147 inhibited the growth of HepG2 ( $\left.\mathrm{IC}_{50}: 4.3 \mu \mathrm{M}\right)$ and MDA-MB-231 cells $\left(\mathrm{IC}_{50}: 19.9 \mu \mathrm{M}\right)$, whereas metabolite 150 demonstrated $\mathrm{IC}_{50}$ values of 40.7 and $40.0 \mu \mathrm{M}$, respectively (Table 2). Compounds 149 and 150 were inactive under these conditions [28]. In 2012, Cottiglia et al. [63] reported myrtucommulone K (149a) from M. communis and based on NMR data, the authors confirmed that the structure of 149 is identical to myrtucommulone K (149a).

Frutescone A-G [(151-156), (+)-157 and (-)-157] were obtained from Baeckea frutescens L. and were shown to possess chemical structures similar to compounds 147-149. Compounds 151-156 bear a triketone coupled with the sesquiterpene, viz., caryophyllene while compound 150 has humulene as the sesquiterpene core (Figure 17) [64]. Compounds 151 and 154 displayed anticancer activity against Caco-2 with $\mathrm{IC}_{50}$ values of $8.08 \mu \mathrm{M}$ and 10.20 $\mu \mathrm{M}$, respectively, whereas, compound 155 inhibited the growth of Caco-2 and A549 cell lines with $\mathrm{IC}_{50}$ values of $7.96 \mu \mathrm{M}$. The other metabolites were only weakly active (Table 2), but all were inactive against HepG2 cells [64]. 


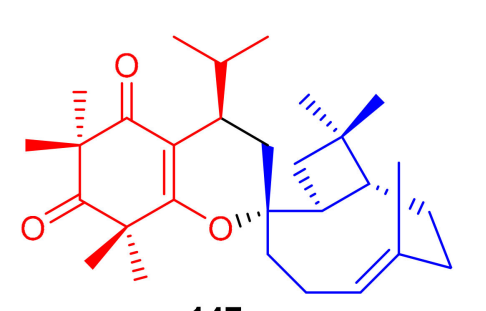

147

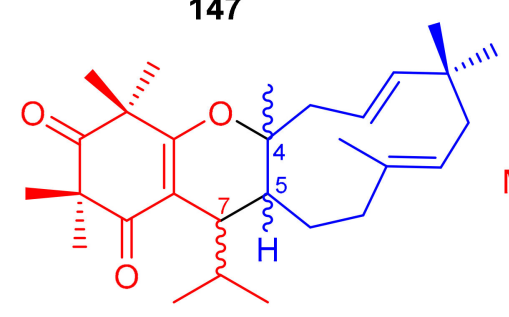

(+)-150: 4S, 5R, 7'S (-)-150: 4R, 5S, 7'R

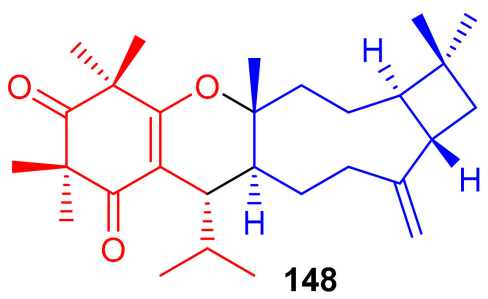

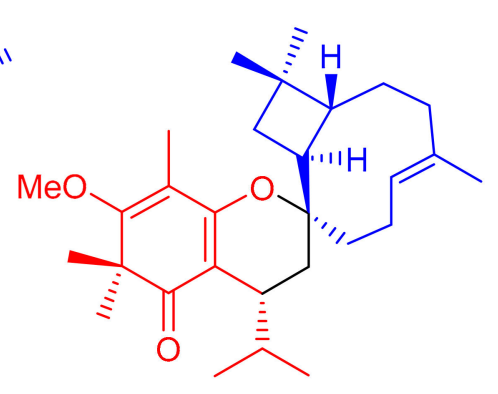

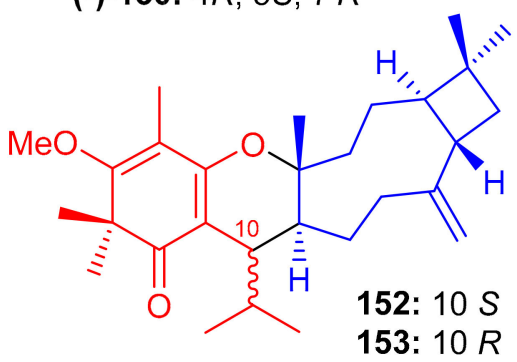<smiles></smiles>

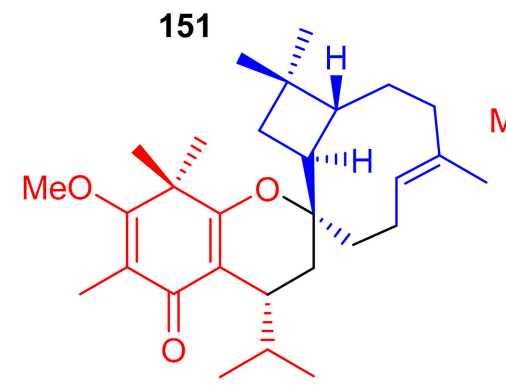

154

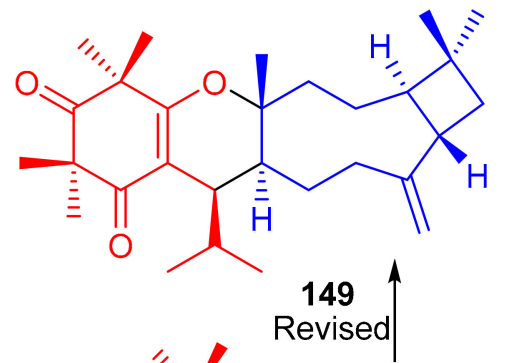<smiles>C=C1CC[C@]2(C)OC3=C(C(=O)C(C)(C)C(=O)C3(C)C)[C@H](C(C)C)[C@]2(C)[C@H]1C1CCCC1(C)C</smiles>

$149 a$

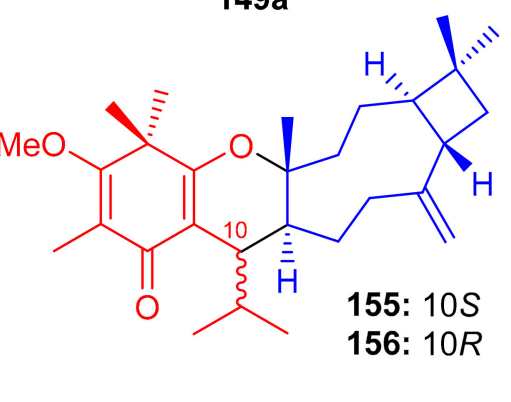

(+)-157: 10S, 4'S, 5'R

(-)-157: 10R, 4'R, 5'S

Figure 17. Structures of syncarpic acid/ $\beta$-triketones-based meroterpenes 147-157.

Triketone-caryophyllene-based meroterpenoids isolated from Rhodomyrtus tomentosa were identified as rhodomyrtials A and B (158 and 159), rhodomentone A (160) and tomentodiones A-D (161-164) (Figure 18) and all were evaluated for their inhibitory potential on tumor metastasis. Compound $\mathbf{1 6 1}$ has a unique 1-oxaspiro[5,8]tridecane core bearing two units of triketone. Biological evaluation demonstrated that only compound $\mathbf{1 6 4}$ displayed significant metastatic effects towards DLD-1 cells. Since no study has been carried out on the mode of action, it should be mentioned that the stereochemistry at C-7 could play an important role in the activity [65].

Zhang et al. [66] separated the tomentodiones E-M (165-173) (Figure 18) from an extract of Rhodomyrtus tomentosa. It was hypothesized that compounds 165-173 could form via a Diels-Alder reaction between triketone and three appropriate terpenes viz., $\beta$-calacorene, (+)-sabinene, and myrcene [66]. Since compounds 165, $( \pm)-\mathbf{1 6 8},( \pm)-\mathbf{1 6 9}$ and 170-173 were non-cytotoxic towards doxorubicin-resistant human breast carcinoma cells (MCF-7/DOX), and compound (+)-168 exhibited a significant potentiation effect by 16.5 reversal fold for MCF-7/DOX, activity was also observed for (+)-169 (10.1 fold), (-)169 (7.4 fold), 173 (5.6 fold), (-)-168 (4.7 fold), and 169 (4.5 fold). Comparison of the activity and structural features of these compounds, suggests that the stereochemistry at C-7' might be significant in determining and enhancing biological effects [66]. 


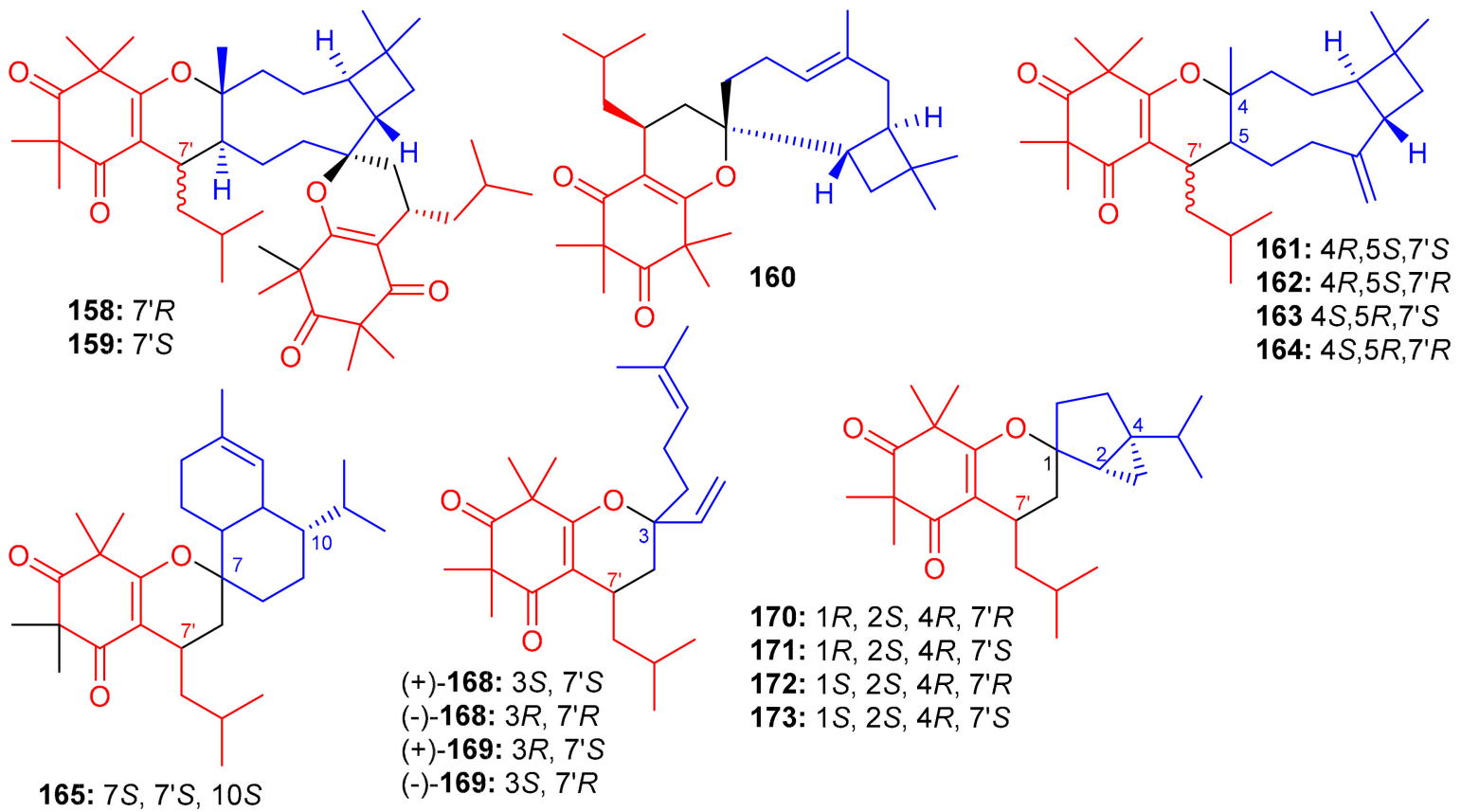

166: $7 R, 7^{\prime} R, 10 S$

167: $7 R, 7 ' S, 10 S$

Figure 18. Structures of syncarpic acid/ $\beta$-triketones-based meroterpenes 158-173.

Callisalignenes G-I (174-176) (Figure 19), isolated from the medicinal plant Callistemon salignus, illustrate $\beta$-triketone and monoterpene moieties in their structures. Spectroscopic analysis and CD calculations revealed that compounds 175 and 176 also possess a secbutyl moiety at C-7, which is not so common in natural products. The three metabolites inhibited the growth of HCT116 cancer cell lines with $\mathrm{IC}_{50}$ values of $8.51,9.12$ and $16.33 \mu \mathrm{M}$, respectively (Table 2). This activity has been reported to be even better than the positive control (VP-16, $20.26 \mu \mathrm{M})$. Compounds 174 and 176 also exhibited cytotoxicity against A549 cell lines with $\mathrm{IC}_{50}$ values of 12.85 and $10.03 \mu \mathrm{M}$, respectively, which is also better than VP-16 $\left(\mathrm{IC}_{50}=25.79 \pm 6.2 \mu \mathrm{M}\right)$, respectively [67]. 
Table 2. Syncarpic acid/ $\beta$-triketones-based meroterpenes.

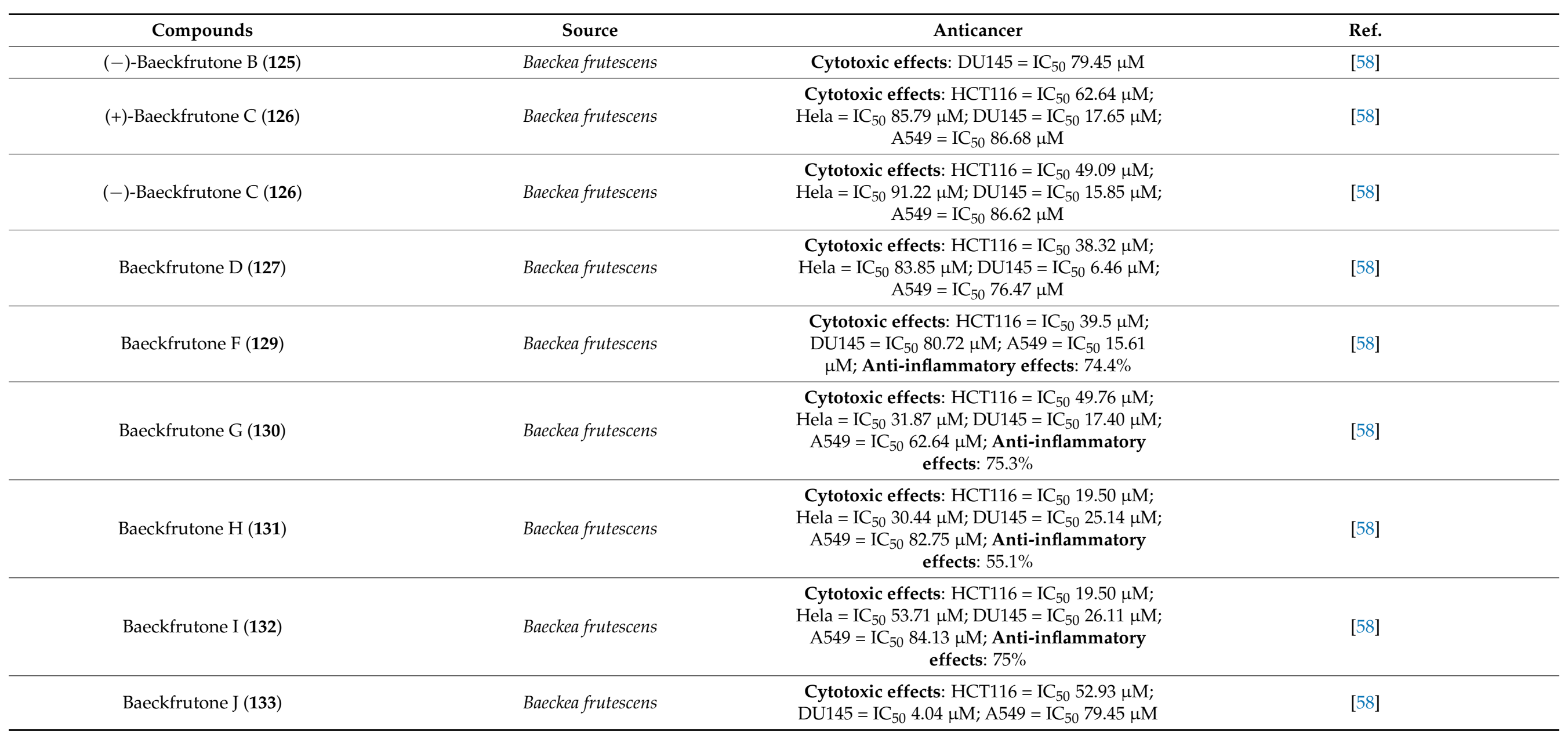


Table 2. Cont.

\begin{tabular}{|c|c|c|c|}
\hline Compounds & Source & Anticancer & Ref. \\
\hline Baeckfrutone K (134) & Baeckea frutescens & $\begin{array}{c}\text { Cytotoxic effects: HCT116 = IC } 5012.89 \mu \mathrm{M} \\
\text { DU145 = IC } 5 \text {;0 } 77.06 \mu \mathrm{M} ; \mathrm{A} 549=\mathrm{IC}_{50} 80.11 \mu \mathrm{M}\end{array}$ & [58] \\
\hline Baeckfrutone L (135) & Baeckea frutescens & $\begin{array}{c}\text { Cytotoxic effects: HCT116 = IC } \mathrm{IC}_{50} 16.48 \mu \mathrm{M} ; \\
\text { Hela = IC } \mathrm{IC}_{50} 19.81 \mu \mathrm{M} ; \mathrm{DU} 145=\mathrm{IC}_{50} 10.0 \mu \mathrm{M} \\
\text { A549 }=\mathrm{IC}_{50} 88.81 \mu \mathrm{M}\end{array}$ & [58] \\
\hline Hyperjaponol H (143) & Hypericum japonicum & Antiviral effects: $\mathrm{EBV}=\mathrm{EC}_{50} 25.0 \mu \mathrm{M}$ & {$[61]$} \\
\hline Tomentosenol A (144) & Rhodomyrtus tomentosa & $\begin{array}{c}\text { Cytotoxic effects: MCF-7 = IC } 508.66 \mu \mathrm{M} ; \\
\text { NCI-H460 = IC } C_{50} 8.62 \mu \mathrm{M} ; \mathrm{SF}-268=\mathrm{IC}_{50} 10.01 \\
\mu \mathrm{M} ; \mathrm{HepG}-2=\mathrm{IC}_{50} 9.44 \mu \mathrm{M}\end{array}$ & [61] \\
\hline Myrtucommulone (147) & Myrtus communis & $\begin{array}{c}\text { Cytotoxic effects: HepG2 }=\mathrm{IC}_{50} 4.39 \mu \mathrm{M} ; \\
\text { MDA-MB-231 }=\mathrm{IC}_{50} 19.92 \mu \mathrm{M}\end{array}$ & [62] \\
\hline Myrtucommulone (148) & Myrtus communis & $\begin{array}{c}\text { Cytotoxic effects: HepG2 }=\mathrm{IC}_{50} 40.7 \mu \mathrm{M} ; \\
\text { MDA-MB-231 }=\mathrm{IC}_{50} 40.0 \mu \mathrm{M}\end{array}$ & [62] \\
\hline Frutescone A (151) & Baeckea frutescens & $\begin{array}{c}\text { Cytotoxic effects: Caco-2 }=\mathrm{IC}_{50} 8.08 ; \mathrm{A} 549= \\
\mathrm{IC}_{50} 20.07 \mu \mathrm{M}\end{array}$ & {$[64]$} \\
\hline Frutescone C (153) & Baeckea frutescens & $\begin{array}{c}\text { Cytotoxic effects: Caco-2 = } \mathrm{IC}_{50} 14.83 \mu \mathrm{M} ; \\
\text { A549 = } \mathrm{IC}_{50} 27.74 \mu \mathrm{M}\end{array}$ & [64] \\
\hline Frutescone D (154) & Baeckea frutescens & $\begin{array}{c}\text { Cytotoxic effects: Caco-2 = } \mathrm{IC}_{50} 10.20 \mu \mathrm{M} ; \\
\text { A549 = } \mathrm{IC}_{50} 26.25 \mu \mathrm{M}\end{array}$ & {$[64]$} \\
\hline Frutescone E (155) & Baeckea frutescens & $\begin{array}{c}\text { Cytotoxic effects: Caco-2 }=\mathrm{IC}_{50} 7.96 \mu \mathrm{M} ; \\
\text { A549 }=\mathrm{IC}_{50} 5.55 \mu \mathrm{M}\end{array}$ & {$[64]$} \\
\hline Frutescone F (156) & Baeckea frutescens & $\begin{array}{c}\text { Cytotoxic effects: Caco-2 }=\mathrm{IC}_{50} 16.51 \mu \mathrm{M} ; \\
\text { A549 }=\mathrm{IC}_{50} 39.02 \mu \mathrm{M}\end{array}$ & {$[64]$} \\
\hline ( \pm )-Frutescone G (157) & Baeckea frutescens & $\begin{array}{c}\text { Cytotoxic effects: Caco-2 = IC } \mathrm{I}_{50} 14.31 \mu \mathrm{M} ; \\
\text { A549 = } \mathrm{IC}_{50} 25.71 \mu \mathrm{M}\end{array}$ & [64] \\
\hline Callisalignene G (174) & Callistemon salignus & $\begin{array}{c}\text { Cytotoxic effects: HCT116 = } \mathrm{IC}_{50} 8.51 \mu \mathrm{M} ; \\
\text { A549 }=\mathrm{IC}_{50} 12.85 \mu \mathrm{M}\end{array}$ & [67] \\
\hline
\end{tabular}


Table 2. Cont

\begin{tabular}{|c|c|c|c|}
\hline Compounds & Source & Anticancer & Ref. \\
\hline Callisalignene H (175) & Callistemon salignus & Cytotoxic effects: HCT116 $=\mathrm{IC}_{50} 9.12 \mu \mathrm{M}$ & [67] \\
\hline Callisalignene I (176) & Callistemon salignus & $\begin{array}{l}\text { Cytotoxic effects: } \mathrm{HCT} 116=\mathrm{IC}_{50} 16.33 \mu \mathrm{M} ; \mathrm{A} 549=\mathrm{IC}_{50} \\
10.03 \mu \mathrm{M}\end{array}$ & [67] \\
\hline Frutescone I (178) & Baeckea frutescens & Anti-inflammatory effects: NO production $=\mathrm{IC}_{50} 18.75 \mu \mathrm{M}$ & [68] \\
\hline Frutescone L (179) & Baeckea frutescens & Anti-inflammatory effects: NO production $=\mathrm{IC}_{50} 30.54 \mu \mathrm{M}$ & [68] \\
\hline Frutescone M (180) & Baeckea frutescens & Anti-inflammatory effects: $\mathrm{NO}$ production $=\mathrm{IC}_{50} 15.17 \mu \mathrm{M}$ & [68] \\
\hline ( \pm )-Compound (181) & Baeckea frutescens & Anti-inflammatory effects: $\mathrm{NO}$ production $=\mathrm{IC}_{50} 1.80 \mu \mathrm{M}$ & [68] \\
\hline Compound (182) & Baeckea frutescens & Anti-inflammatory effects: $\mathrm{NO}$ production $=\mathrm{IC}_{50} 0.36 \mu \mathrm{M}$ & [68] \\
\hline Compound (183) & Baeckea frutescens & Anti-inflammatory effects: $\mathrm{NO}$ production $=\mathrm{IC}_{50} 3.70 \mu \mathrm{M}$ & [68] \\
\hline$( \pm)$-Compound (184) & Baeckea frutescens & Anti-inflammatory effects: NO production $=\mathrm{IC}_{50} 2.07 \mu \mathrm{M}$ & [68] \\
\hline ( \pm )-Compound (185) & Baeckea frutescens & Anti-inflammatory effects: NO production $=\mathrm{IC}_{50} 6.50 \mu \mathrm{M}$ & [68] \\
\hline Baefrutone A (188) & Baeckea frutescens & Anti-inflammatory effects: NO Production $=\mathrm{IC}_{50} 9.15 \mu \mathrm{M}$ & [69] \\
\hline Baefrutone B (189) & Baeckea frutescens & Anti-inflammatory effects: NO Production $=\mathrm{IC}_{50} 17.73 \mu \mathrm{M}$ & [69] \\
\hline Baefrutone D (191) & Baeckea frutescens & Anti-inflammatory effects: NO Production $=\mathrm{IC}_{50} 18.04 \mu \mathrm{M}$ & [69] \\
\hline hyperjaponols A (194a) & Hypericum japonicum & Antiviral effects: $\mathrm{EBV}=\mathrm{EC}_{50} 10.33 \mu \mathrm{M}$ & [70] \\
\hline Hyperjaponol B (195a) & Hypericum japonicum & Antiviral effects: $\mathrm{EBV}=\mathrm{EC}_{50} 0.57 \mu \mathrm{M}$ & [70] \\
\hline Hyperjaponol B (195b) & Hypericum japonicum & Antiviral effects: $\mathrm{EBV}=\mathrm{EC}_{50} 6.60 \mu \mathrm{M}$ & [70] \\
\hline Hyperjaponol D (197) & Hypericum japonicum & Antiviral effects: $\mathrm{EBV}=\mathrm{EC}_{50} 0.49 \mu \mathrm{M}$ & [70] \\
\hline
\end{tabular}




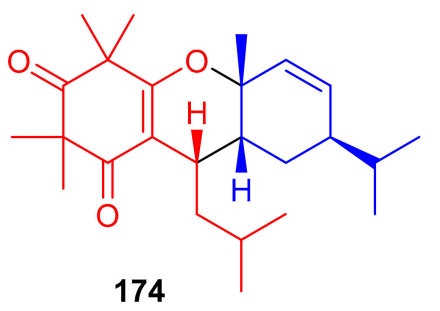

174

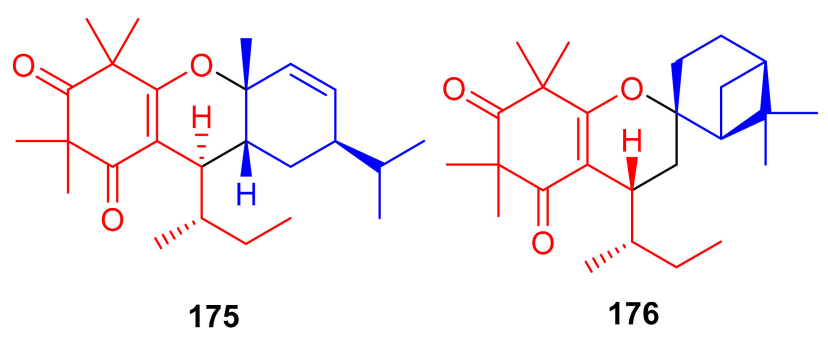

175

Figure 19. Structures of syncarpic acid/ $\beta$-triketones-based meroterpenes 174-176.

The small library of compounds 177-187 (Figure 20) were separated from Baeckea frutescens and identified through spectroscopic analyses, ECD calculations and X-ray crystallography. Metabolites 177-187 could form between the common triketone (tasmanone) sesquiterpenes/monoterpenes viz., bicyclosequiphellandrene (compounds 145-147), $\beta$-caryophyllene (compound 148), $\beta$-cubebene (compounds 181 and 182), (-)-sabinene (compounds 183 and 184), $\beta$-pinene (compound 185), and myrcene (compounds 186 and 187) [68].<smiles>COC1=C(C)C(=O)C2=C(O[C@@]3(C=C4[C@@H](C(C)C)CC[C@H](C)[C@H]4CC3)C[C@H](C(C)C)C2(C)C)C(C)(C)C(OC)=C(C)C1=O</smiles><smiles>C=C(CC[C@]1(C)OC2=C(C(=O)C(C)=C(OC)C2(C)C)[C@H]1C(C)C)[C@H]1CC(C)(C)[C@H]1C</smiles><smiles>COC1=C(C)C(=O)C2=C(O[C@]3(CC[C@H]4[C@@H](C)CC[C@H](C(C)C)[C@H]4[C@H]3C(C)C)CC2C(C)C)C1(C)C</smiles><smiles></smiles><smiles>COC1=C(C)C2=C(C(=O)C1(C)C)[C@@H](C(C)C)C[C@@]1(CCC(C(C)C)(C(C)C)[C@@H]1C)O2</smiles>

(+)-183: 10R, 1'S, 2'S, 4'R $(-)-183: 10 S, 1^{\prime} R, 2^{\prime} R, 4^{\prime} S$<smiles>COC1=C(C)C2=C(C(=O)C(C)(C)C(C)(C)O2)[C@H](C(C)C)C[C@]12CCC1(C(C)C)C[C@@H]12</smiles><smiles>COC1=C(C)C(=O)C2=C(O[C@]3(CC[C@H]4CC[C@H]3C4(C)C)C[C@@H]2C(C)C)C1(C)C</smiles><smiles>CCCCC(CC)C(C)C</smiles>

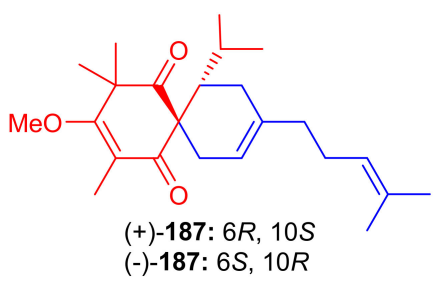

$(-)-186: 6 R, 10 R$

Figure 20. Structures of syncarpic acid/ $\beta$-triketones-based meroterpenes 177-187.

Among other antioxidant meroterpenoids, frutescones H-R (177-182) only moderately inhibited the NO production in LPS-induced RAW 264.7 cells with $\mathrm{IC}_{50}$ values in the range of 15.17-50.0 $\mu \mathrm{M}$ (Table 2), whereas, compounds 183-187 exhibited significant IC $_{50}$ values $(0.36-6.50 \mu \mathrm{M})$ [68]. These compounds are more potent inhibitors of NO production than the standard $N$-monomethyl-L-arginine (L-NMMA, $\mathrm{IC}_{50}=30.92 \mu \mathrm{M}$ ). Since compound 184 showed the highest anti-inflammatory potential $\left(\mathrm{IC}_{50}=0.36 \mu \mathrm{M}\right)$, it was further evaluated against LPS-induced upregulation of TNF- $\alpha$ and IL- 6 . A review of the activity level and structural features of compounds 177-187 revealed that the overall structure of ring A 
plays a more important role in the potential of these compounds, especially the position of the double bonds and keto groups [68].

Baefrutones A-F (188-193) (Figure 21) bearing a rather rare triketone-phloroglucinol unit coupled to sesquiterpene/monoterpene skeletons were isolated from Baeckea frutescens. Interestingly, in compounds 188-191, the triketone-phloroglucinol core was attached to $\alpha$-thujene while meroterpenoids 192 and 193 have a $\beta$-caryophyllene framework instead of the $\alpha$-thujene. Moreover compounds 188-191 displayed anti-inflammatory effects towards NO production with $\mathrm{IC}_{50}=9.1$ to $18.0 \mu \mathrm{M}$ (Table 2), while meroterpenoids 192 and 193 were not active. Notably compounds $\mathbf{1 8 8}-\mathbf{1 9 1}$ were more potent than the positive control L-NMMA $\left(\mathrm{IC}_{50}=30.9 \mu \mathrm{M}\right)[69]$.<smiles>COC1=C(C)C(=O)C([C@@H](c2c(O)c(C(=O)C(C)C)c(O)c3c2O[C@]2(C)[C@@H](C)C[C@H]2C[C@@H]3C(C)C)C(C)C)=C(O)C1(C)C</smiles><smiles>COC1=C(C)C(=O)C([C@@H](c2c(O)c(C(=O)C(C)C)c(O)c3c2O[C@]2(C)[C@@H](C)C[C@]4(C(C)C)C[C@@]42[C@@H]3C)C(C)C)=C(O)C1(C)C</smiles>

189<smiles>COC1=C(C)C(=O)C([C@H](c2c(O)c3c(c(C(=O)C(C)C)c2O)O[C@]2(C)[C@@H](C3)C[C@@]3(C(C)C)C[C@H]32)C(C)C)=C(O)C1(C)C</smiles><smiles>COC(C)=C(C)C</smiles><smiles>CC1=C(C)C(C)=C(O)C(C)(C)C1=CC(C)C</smiles>

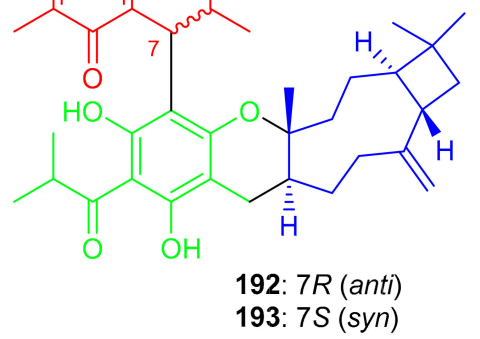<smiles>COC1=C(C)C(=O)C([C@H](c2c(O)c3c(c(C(=O)C(C)C)c2O)O[C@@]2(C)[C@@H](C3)C[C@](C)(C(C)C)[C@H]2C)C(C)C)=C(O)C1(C)C</smiles>

Figure 21. Structures of syncarpic acid/ $\beta$-triketones-based meroterpenes 188-193.

Filicinic acid-based meroterpenoids, the hyperjaponols A-G (194-200) (Figure 22) were isolated from Hypericum japonicum and comprise 6/6/7/5, 6/6/11, or 6/6/10 sized ring frameworks. Moreover compounds 194-196 were reported as enantiomeric pairs and these compounds displayed weak to moderate anti-EBV effects while compound 200 was not active. Enantiomer 194a displayed an $\mathrm{EC}_{50}$ value of $10.33 \mu \mathrm{M}$, while its enantiomer $195 \mathbf{b}$ was weakly active (Table 2). In a similar enantiomeric differentiation, metabolite 195a $\left(\mathrm{EC}_{50}=0.57 \mu \mathrm{M}\right)$ was more active than $163 \mathrm{~b}\left(\mathrm{EC}_{50}=6.60 \mu \mathrm{M}\right)$ (Table 2). Among other isolates, compound 197 exhibited the lowest $\mathrm{EC}_{50}$ value of $0.49 \mu \mathrm{M}$, which is 5-fold lower than the standard drug ganciclovir $\left(\mathrm{EC}_{50} 2.86 \mu \mathrm{M}\right)$ [70].

Meroterpenoids, callisalignenes D-F (201-203) (Figure 23) were produced by Callistemon salignus [47] and based on MIC values [71] these compounds were not active in antimicrobial screening. In another report, meroterpenoids, myrtucomvalones A-C (204-206) were reported from Myrtus communis and compound 206 illustrated moderate antiviral effects towards the respiratory syncytial virus (RSV) with $\mathrm{IC}_{50}: 15.8 \mu \mathrm{M}$ [72]. Liu et al. [73] reported two meroterpenoids, rhodomentones A (207) and B (208) were produced by Rhodomyrtus tomentosa and featured an uncommon caryophyllene-conjugated oxa-spiro[5.8]tetradecadiene core. In addition, Senadeera et al. [74] reported intermediones A-D (209-212) from the tree Corymbia intermedia. Compounds 209, 210, and 212 possessed moderate antiplasmodial effects towards Plasmodium falciparum with $\mathrm{IC}_{50}$ : $12.5,9.9$ to $20.8 \mu \mathrm{M}$, respectively. 


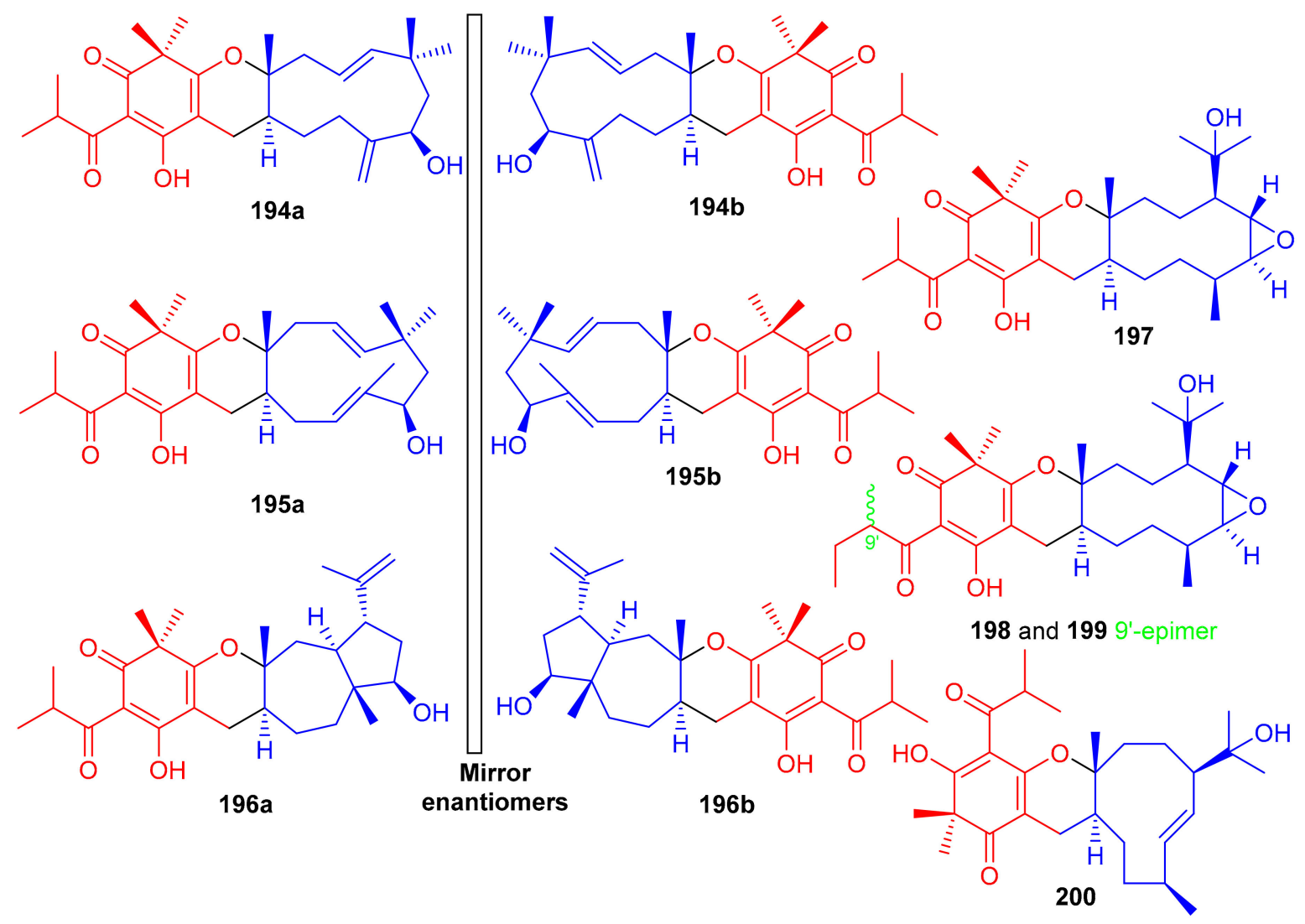

Figure 22. Structures of syncarpic acid/ $\beta$-triketones-based meroterpenes 194-200.
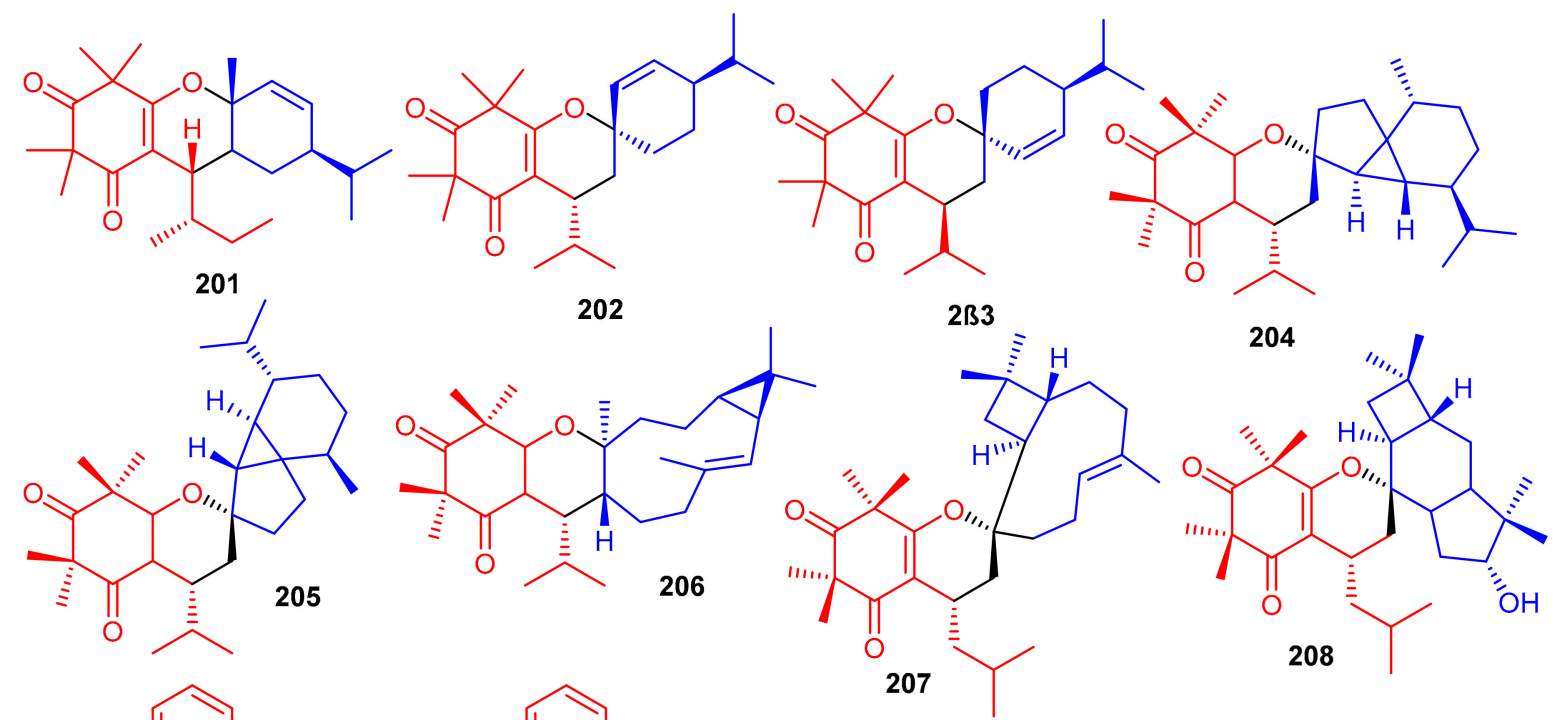

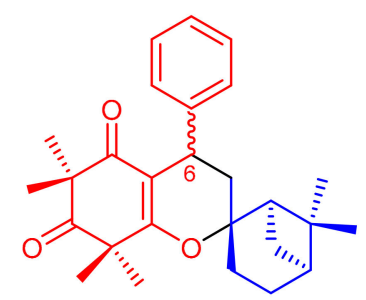

209: $6 \beta$

210: $6 \alpha$<smiles>CC1(C)C(=O)C2=C([C@@H](c3ccccc3)C[C@@]3(CC[C@H]4CC[C@H]3C4(C)C)O2)C(C)(C)C1=O</smiles>

211

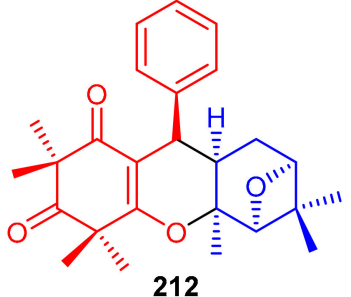

212

Figure 23. Structures of syncarpic acid/ $\beta$-triketones-based meroterpenes 201-212. 
Elodeoidols A-I (213-221) (Figure 24) were isolated from Hypericum elodeoides. Compounds 217, 220 and 221 illustrated moderate antibacterial effects towards Streptococcus mutans, Fusobacterium nucleatum, and Streptococcus sanguis. In addition, compounds 215, 219 and 220 demonstrated potent NO inhibitory effects towards LPS induced RAW264.7 cells with $\mathrm{IC}_{50}$ ranging from 10 to $34 \mu \mathrm{M}$ [75]. Moreover, rhotomentodiones C-E, (222-224) (Figure 24) were produced by Rhodomyrtus tomentosa. Rhotomentodione D (223) demonstrated antibacterial effects towards Propionibacterium acnes (MIC: $12.5 \mu \mathrm{g} / \mathrm{mL}$ ) and AChE inhibitory effects with an $\mathrm{IC}_{50}: 22.9 \mu \mathrm{M}$ [76]. Frutescones S-U (225-227) were isolated from Baeckea frutescens and meroterpene $\mathbf{2 2 5}$ demonstrated potent anti-inflammatory effects with an $\mathrm{IC}_{50}: 0.81 \mu \mathrm{mol} / \mathrm{L}[77]$.

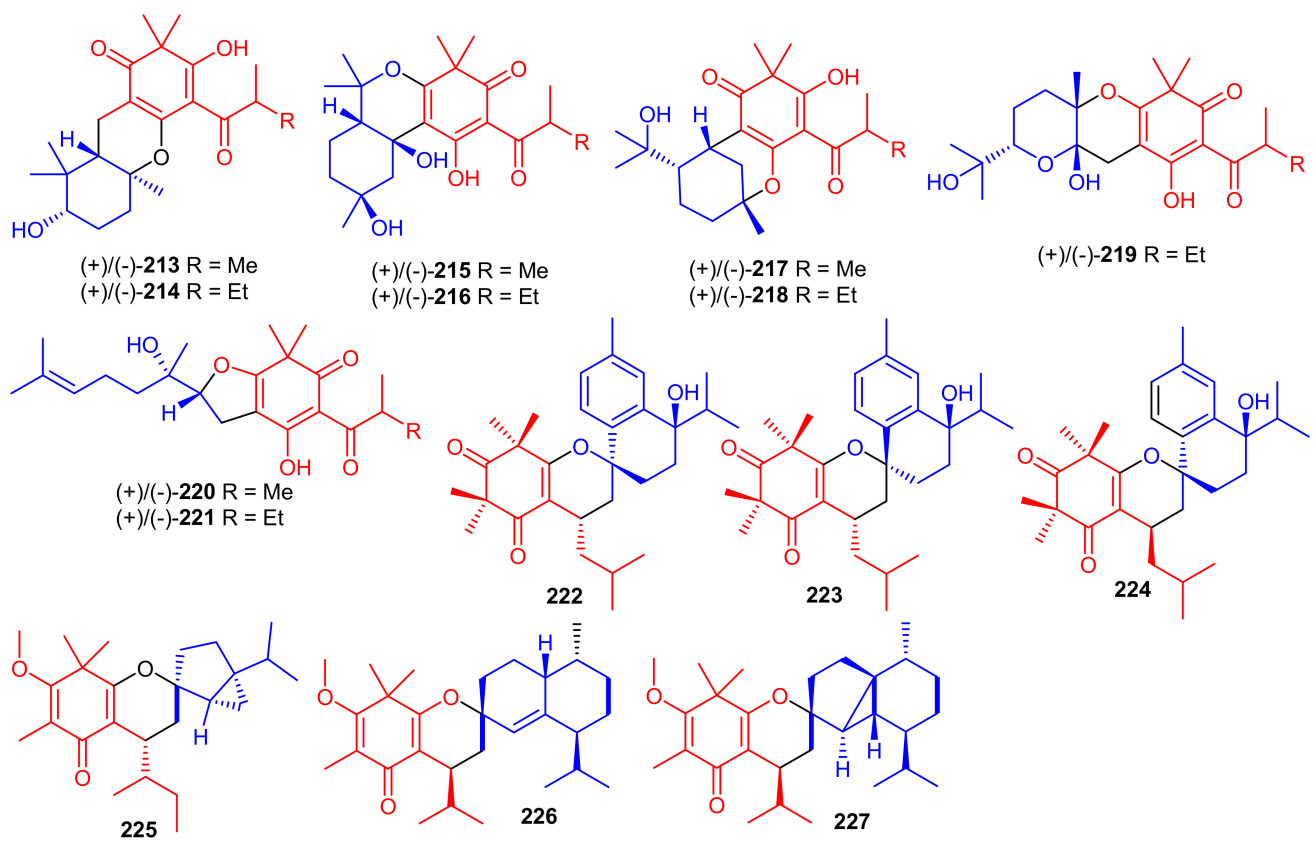

Figure 24. Structures of syncarpic acid/ $\beta$-triketones-based meroterpenes 213-227.

$\beta$-Triketone-based meroterpenes rtomentones A-H (228-235) (Figure 25) were isolated from Rhodomyrtus tomentosa and all compounds were not active towards A549, MDAMB-231, and DLD-1 cancer cells [78]. ( \pm )-Dryocrassoids A-D (236-239) were reported from Dryopteris crassirhizoma. Moreover these compounds exhibited moderate anti-HSV-1 activity with $\mathrm{IC}_{50}$ : ranging from 23.4 to $95.0 \mu \mathrm{M}$. In addition, compounds 236-239 also possessed anti-RSV effects with $\mathrm{IC}_{50}$ : ranging from 11.4 to $50.2 \mu \mathrm{M}$. [50]. 


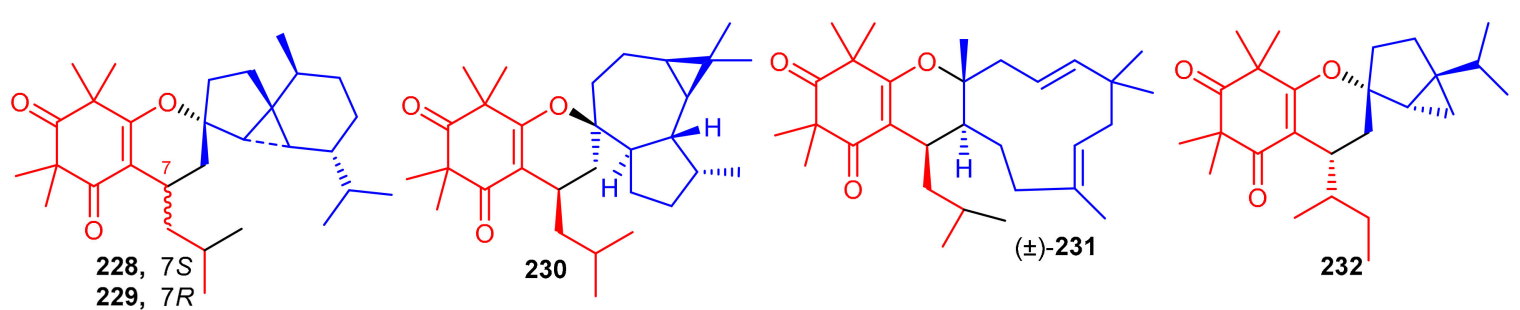

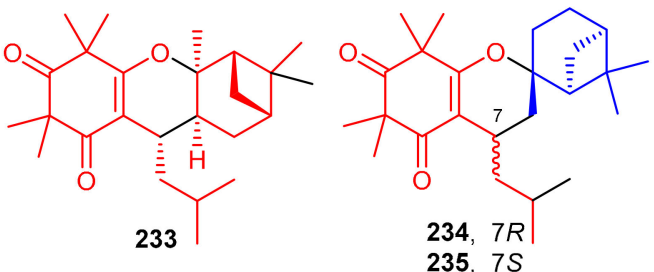

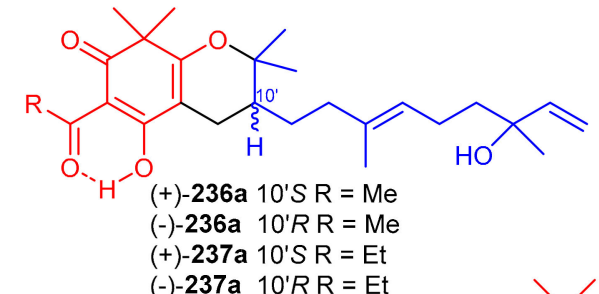

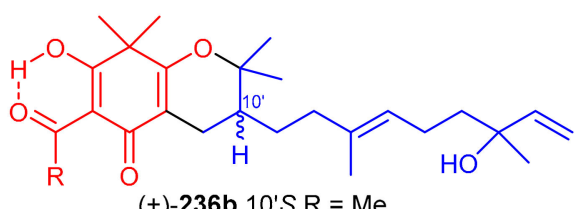

(+)-236b 10'S R = Me

$(-)-236 \mathrm{~b} 10^{\prime} R \mathrm{R}=\mathrm{Me}$

(+)-237b 10'S R = Et

$(-)-237 \mathrm{~b} 10^{\prime} R \mathrm{R}=\mathrm{Et}$

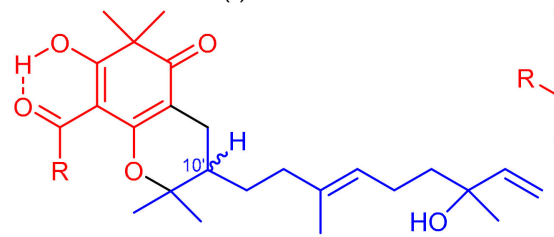

(+)-238 10'S $(-)-23810^{\prime} R$<smiles>[R]C1=C2C(=O)C3=C(O[C@](C)(/C=C\C=C(C)C)[C@@H](C)C3)C(C)(C)C2=C([R])C1=O</smiles>

$(+)-239 b$ 6'S, 7'S

$(-)-239 \mathrm{~b} 66^{\prime} R, 7^{\prime} R$

Figure 25. Structures of syncarpic acid/ $\beta$-triketones-based meroterpenes 228-239.

\section{Alklaoid-Based Meroterpenoids}

Phenazine- and Phyridine-Based Meroterpenoids

Phenazine-derived meroterpenoids, viz., marinocyanins A-F (240-245) (Figure 26) along with lavanducyanin (246) were produced by the marine Actinomycete strains. Compounds $\mathbf{2 4 0 - 2 4 5}$ are unique secondary metabolites comprising the bromo-phenazinone nucleus supplemented by $\mathrm{N}$-isoprenoid moieties or a cyclolavandulyl ring in their structures [79]. Lavanducyanin (246) was re-isolated from Streptomyces sp. as a testosterone $5 \alpha$-reductase inhibitor and was named WS-9659A Quite recently Kohatsu et al. [80] reported the total synthesis of lavanducyanin (246). Marinocyanin A (240) has been reported to be a potent antibiotic, since it potentially inhibited $(\mathrm{MIC}=0.95 \mu \mathrm{M})$ the growth of amphotericin-resistant Candida albicans in vitro, while the other test compounds were reported as only weak inhibitors (Table 3). In addition, marinocyanins A (240) and B (241) illustrated significant in vitro cytotoxic effects towards human colon carcinoma (HCT-116: 240: $\left.\mathrm{IC}_{50}: 0.049 \mu \mathrm{M} ; 170: \mathrm{IC}_{50}: 0.029 \mu \mathrm{M}\right)$. SAR studies showed that the cyclic structure of the terpenoidal part (cyclolavandulyl ring) plays a significant role in the antifungal activity, and that the halogen plays no particular role in the activity [79]. The standard drugs used in these assays were vancomycin ( $\mathrm{MIC}=0.27 \mu \mathrm{M}$ ) for S. aureus and amphotericin B $(\mathrm{MIC}=0.084 \mu \mathrm{M})$ for $C$. albicans. Zhang et al. [81] isolated an unusual C21 pyridine bearing meroterpenoid 247 from the sponge Cacospongia sp. 
<smiles>[R]c1ccc2n(CC3=C(C)C([R4])C([R2])(C)CC3[R])c3ccccc3nc-2c1=O</smiles>

240: $R_{1}=B r, R_{2}=M e, R_{3}=R_{4}=H$

243: $\mathrm{R}_{1}=\mathrm{Br}, \mathrm{R}_{2}=\mathrm{CH}_{2} \mathrm{OH}, \mathrm{R}_{3}=\mathrm{R}_{4}=\mathrm{H}$

244: $R_{1}=B r, R_{2}=M e, R_{3}=H, R_{4}=O H$

245: $R_{1}=B r, R_{2}=M e, R_{3}=O H, R_{4}=H$

246: $R_{1}=H, R_{2}=\mathrm{CH}_{2} \mathrm{OH}, \mathrm{R}_{3}=\mathrm{R}_{4}=\mathrm{H}$

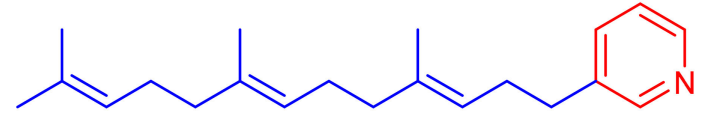

247

Figure 26. Structures of alkaloid-based meroterpenoids 240-247.

Table 3. Alkaloids-based meroterpenoids.

\begin{tabular}{|c|c|c|c|}
\hline Compounds & Source & Anticancer & Ref. \\
\hline Marinocyanin A (240) & Actinomycete strains & $\begin{array}{l}\text { Cytotoxic effects: HCT- } 116=\mathrm{IC}_{50} \\
0.049 \mu \mathrm{M} \text {; Antimicrobial effects: } \\
\text { Candida albicans = MIC } 0.95 \mu \mathrm{M} ; \\
\text { Staphylococcus aureus = MIC } 2.3 \mu \mathrm{M}\end{array}$ & [79] \\
\hline Marinocyanin B (241) & Actinomycete strains & $\begin{array}{c}\text { Cytotoxic effects: HCT- } 116=\mathrm{IC}_{50} \\
0.029 \mu \mathrm{M} ; \text { Antimicrobial effects: } \\
\text { Candida albicans = MIC } 5.79 \mu \mathrm{M} ; \\
\text { Staphylococcus aureus = MIC } 33.92 \mu \mathrm{M}\end{array}$ & [79] \\
\hline Marinocyanin C (242) & Actinomycete strains & $\begin{array}{c}\text { Antimicrobial effects: Candida } \\
\text { albicans }=\text { MIC } 3.90 \mu \mathrm{M} ; \text { Staphylococcus } \\
\text { aureus }=\text { MIC } 30.71 \mu \mathrm{M}\end{array}$ & [79] \\
\hline Marinocyanin D (243) & Actinomycete strains & $\begin{array}{c}\text { Antimicrobial effects: Candida } \\
\text { albicans = MIC } 14.65 \mu \mathrm{M} ; \\
\text { Staphylococcus aureus = MIC } 36.62 \mu \mathrm{M}\end{array}$ & [79] \\
\hline Marinocyanin E (244) & Actinomycete strains & $\begin{array}{c}\text { Antimicrobial effects: Candida } \\
\text { albicans = MIC } 14.65 \mu \mathrm{M} ; \\
\text { Staphylococcus aureus = MIC } 36.62 \mu \mathrm{M}\end{array}$ & [79] \\
\hline Marinocyanin F (245) & Actinomycete strains & $\begin{array}{c}\text { Antimicrobial effects: Candida } \\
\text { albicans = MIC } 14.65 \mu \mathrm{M} ; \\
\text { Staphylococcus aureus = MIC } 36.62 \mu \mathrm{M}\end{array}$ & [79] \\
\hline Lavanducyanin (246) & Streptomyces sp. & $\begin{array}{c}\text { Antimicrobial effects: Candida } \\
\text { albicans = MIC } 114.67 \mu \mathrm{M} ; \\
\text { Staphylococcus aureus }=\text { MIC } 56.93 \mu \mathrm{M}\end{array}$ & {$[79]$} \\
\hline
\end{tabular}

\section{Sesquiterpene-Based Meroterpenoids}

The Vietnamian marine sponge Spongia sp. produces a range of meroterpenoids viz., langcoquinone A (248) and B (249) (Figure 27). On the other hand, compounds 248 and 249 were inactive against K. pneumoniae and E. coli, compared to the positive control Kanamycin ((MIC $=6.25$ and $12.5 \mu \mathrm{M}$, respectively) [82]. 
<smiles>[R]C1=CC(=O)C(O)=C(C[C@@]2(C)[C@@H](C)CC[C@]3(C)C(=C)CCC[C@H]32)C1=O</smiles>

248: $\mathrm{R}=\mathrm{NHCH}_{3}$ 249: $\mathrm{R}=\mathrm{NHCH}_{2} \mathrm{CH}_{2} \mathrm{SCH}_{3}$<smiles>[R]c1c(C(=O)OC)cc(C[C@]2([R7])[C@@H](C)CC[C@]3(C)C(=C)CCC[C@@H]32)c(O)c1[R2]</smiles>

250: $\mathrm{R}_{1}=\mathrm{Me}, \mathrm{R}_{2}=\mathrm{OMe}, \mathrm{R}_{3}=\mathrm{OH}$

251: $\mathrm{R}_{1}=\mathrm{CHO}, \mathrm{R}_{2}=\mathrm{OMe}, \mathrm{R}_{3}=\mathrm{H}$

252: $\mathrm{R}_{1}=\mathrm{CHO}, \mathrm{R}_{2}=\mathrm{OMe}, \mathrm{R}_{3}=\mathrm{OH}$<smiles></smiles>

253

Figure 27. Structures of sesquiterpene-based meroterpenoids 248-253.

In another investigation, Nguyen et al. [83] further isolated sesquiterpene-based meroterpenoids, langconols A-C (250-252) (Figure 27) and langcoquinone C (253) from the same sponge viz., Spongia sp. Furthermore, compounds 250-252 bear the 4,9-friedodrimane skeleton along with phenolic functionality while langcoquinone C (253) has an hydroxyquinone instead of the phenolic group. Compound 253 exhibited significant inhibitory activity (MIC $=6.25 \mu \mathrm{M})$ against $B$. subtilis and $S$. aureus, with the same potential as mentioned above for the reference drug ampicillin, whereas, compounds 250 and 253 only inhibited the growth of $B$. subtilis with MICs of 12.5 and $25.0 \mu \mathrm{M}$, respectively. Compound $\mathbf{2 5 0}$ has good potential to be an antibacterial and non-toxic agent and thus offers itself as a strong candidate to be studied for the development of a potentially new antibiotic [83].

The marine sponge Dysidea sp. produces the sesquiterpene-based meroterpenoids dysidphenols A-C (254-256), along with smenospongimine (257), (Figure 28) all of which were characterized by spectroscopic analyses and ECD calculations [84]. Moreover compounds 254-256 all comprise a drimane-type sesquiterpene unit attached to a phenolic entity through either an oxaspiro center or methylene linkage. On the other hand, compound 267 comprises the 4,9-friedodrimane skeleton attached to hydroxybenzoquinone moieties. Compounds 254 and 256 were weakly active against E. coli, B. subtilis and $S$. aureus. However, the other test compounds 257 was found to be more potent against these three bacterial species with MIC values between 3.1 and $12.5 \mu \mathrm{g} / \mathrm{mL}$ (Table 4).<smiles>COC(=O)c1cc(O)c2c(c1)[C@]1(C)[C@@H](C)CC[C@H]3C(C)(C)CCC[C@]31C2</smiles>

254

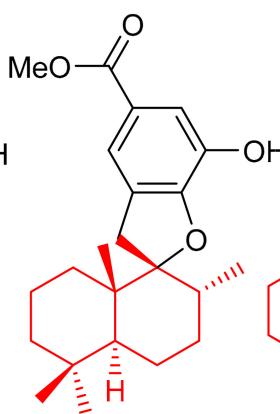

255<smiles>C=C1CCC[C@@H]2[C@@H](C[Pb])[C@@]1(C)CC[C@H](C)[C@]2(C)CC1=C(O)C(=O)C=C(NC)C1=O</smiles>

256
257

Figure 28. Structures of sesquiterpene-based meroterpenoids 254-257.

The marine sponge Dactylospongia sp. has been reported to produce several meroterpenoids including dactylospongins A-D (258-261), melemeleones C-E (262-264), dysidaminone N (265) and 19-O-methylpelorol (266). Compounds 258-265 (Figure 29) have a sesquiterpene moiety attached to either a benzothiazole, phenolic, or benzoquinone core through a C-C bond [85]. An interesting feature of meroterpenoids $\mathbf{2 5 8}$ and $\mathbf{2 5 9}$ is that they comprise a unique thiazole ring which biogenetically, could be derived from cysteine [86]. 
<smiles>C=C1CCC[C@@H]2Oc3cc4ncsc4cc3C[C@@]1(C)CC[C@@H](C)[C@]2(C)Cc1cc2scnc2cc1O</smiles><smiles></smiles><smiles>C=C1CCC[C@@]2(C)C(=C)CCC[C@@H]1[C@H]2CC(=O)O</smiles>

Figure 29. Structures of sesquiterpene-based meroterpenoids 258-266.

Among other meroterpenoid inhibitors of PTP1B, nakijinol G (267) (Figure 30) inhibited the activity of this enzyme with an $\mathrm{IC}_{50}$ value of $4.8 \mu \mathrm{M}$ (Table 4). However, the other metabolites, nakijinol F (268), hyrtiolacton A (269) all isolated from the same marine sponge Hyrtios sp. were inactive. Moreover, none of these compounds were active towards HepG2, RPMI-8226, HeLa, and HL-60 cancer cells. Compounds 267 and 268 have a sesquiterpene coupled to a benzoxazole moiety while in compound 269 the benzoxazole ring is replaced by an $\alpha$-pyrone and benzoquinone unit respectively [87]. Another sponge, Dysidea villosa also produces some unusual meroterpenoids described as dysivillosins A-D (270-273), which all inhibited the release of $\beta$-hexosaminidase with $\mathrm{IC}_{50}$ ranging from 8.2 to $19.9 \mu \mathrm{M}$ (Table 4). In addition, compounds $\mathbf{2 7 0 - 2 7 3}$ exert a positive inhibitory effect on LTB-4 and IL4 and compound 270 potentially inhibited the activation of Syk. It may thus be concluded that this meroterpenoid could potentially be a new chemotherapeutic scaffold targeting Syk-associated allergies [88]. Dysivillosins A-D (270-273) (Figure 30) are meroterpenes bearing a terpene-polyketide-pyridine system and this type of combination is very rare among meroterpenoids. Moreover, meroterpenoids 270-273 have a 2-pyridone core which could be produced biogenetically from L-lysine through amidation, decarboxylation, and dehydrogenation reactions [89]. 


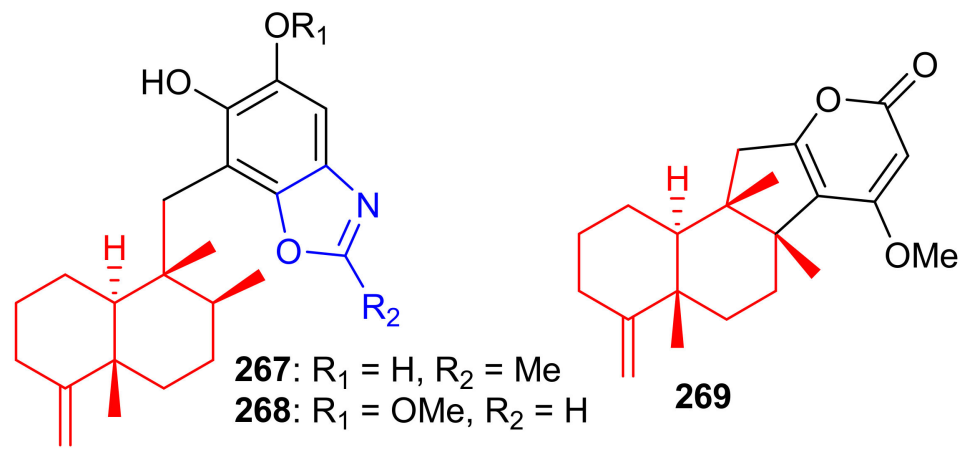<smiles>C=C1CCC[C@H]2[C@]1(C)CC[C@@H](C)[C@@]2(C)Cc1cc(O)c(-c2ccc[nH]c2=O)c(O)c1</smiles><smiles></smiles>

Figure 30. Structures of sesquiterpene-based meroterpenoids 267-273.

Chartarolides A-C (274-276) (Figure 31), the secondary metabolites of the sponge Niphates recondite, were tested for their cytotoxic properties against HCT-116, BGC-823, HepG2, A2780 NCI-H1650, and MCF7 cancer cells. Compound 274 displayed the most potent effects with $\mathrm{IC}_{50}$ values ranging from 1.3 to $1.9 \mu \mathrm{M}$, followed by compound $275\left(\mathrm{IC}_{50}=\right.$ 1.6-4.8 $\mu \mathrm{M}$ ) (Table 4), while compound 276 has been reported to be the least active with $\mathrm{IC}_{50}$ values in the range of 5.4-12.5 $\mu \mathrm{M}$ (Table 4) [90]. However, the activities of these metabolites are lower than the reference drug taxol, which displayed $\mathrm{IC}_{50}$ values of 0.001 to 0.07 against these cell lines. In addition, compounds 274-276 have also been reported as inhibitors $\left(\mathrm{IC}_{50}=2.6-21 \mu \mathrm{M}\right)$ of FGFR3, IGF1R and PDGFRb [90], which is lower than the activity of the positive control satratoxin $\mathrm{H}\left(\mathrm{IC}_{50}=0.05 \mu \mathrm{M}\right)$. Another marine sponge, Dysidea arenaria produces dysiarenone (277) and this compound displayed inhibitory activities towards COX-2 expression with an $\mathrm{IC}_{50}$ value of $6.4 \mu \mathrm{M}$. Compound 277 is a dimeric C-21 meroterpenoid featuring a unique 2-oxaspiro(bicyclo[3.3.1]nonane-9, $1^{\prime}$-cyclopentane) carbon skeleton [91]. This compound reduced the production of PGE2 with IC $\mathrm{I}_{50}: 6.4 \mu \mathrm{M}$ and was $~ 10$ times more potent than that of the positive control avarol [91].

Meroterpenoids 278-281 (Figure 32) were produced by the sponge Dysidea sp. [84] and their absolute configuration were determined via $\mathrm{CD}$ and ECD calculations [92]. In another report, Dysidea sp. also produced meroterpenoids, dysiherbols A-C (282-284) featuring a 6/6/5/6-fused core and dysideanone E (285). Moreover, compounds 282-284 illustrated potent NF- $\mathrm{kB}$ inhibition with $\mathrm{IC}_{50}$ ranging from $0.49-6.4 \mu \mathrm{M}$. Notably, compound 282 was potent towards myeloma cancer (NCI H-929: $\left.\mathrm{IC}_{50}: 0.58 \mu \mathrm{M}\right)$ as well as a potent NF- $\mathrm{KB}$ inhibitor with $\left.\mathrm{IC}_{50}=0.49 \mu \mathrm{M}\right)$ [93]. 


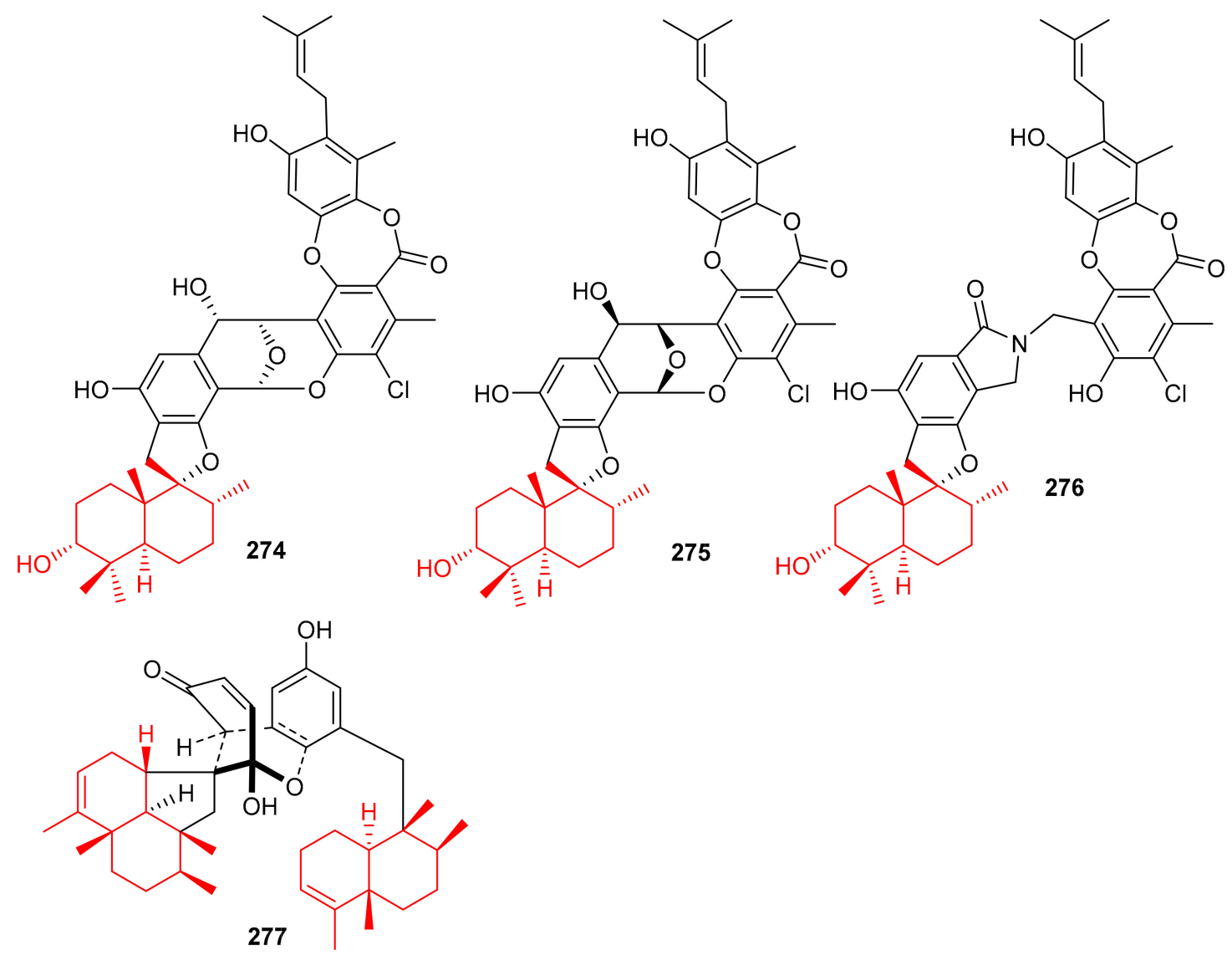

Figure 31. Structures of sesquiterpene-based meroterpenoids 274-277.

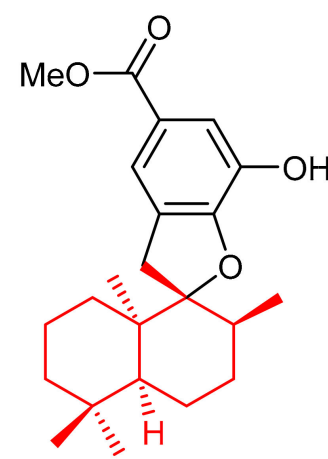

278

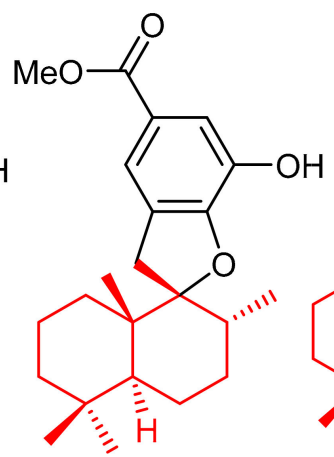

279<smiles>C=C1CCC[C@H]2[C@](C)(CC[C@@H](C)[C@@]2(C)CC2=C(O)C(=O)C=C(NC)C2=O)[C@@H](C)CCC1(C)O</smiles>

280<smiles>C[C@H]1CC[C@]2(C)[C@@](C)(O)CCC[C@]23c2c(O)ccc(O)c2C[C@]13C</smiles>

282<smiles>C[C@@H]1CC[C@]2(C)[C@@](C)(O)[C@@H](O)CC[C@]23c2c(O)ccc(O)c2C[C@]13C</smiles>

283<smiles>C[C@H]1CC[C@@]2(C)[C@@]3(CCC(=O)[C@]2(C)O)c2c(O)ccc(O)c2C[C@]13C</smiles>

284<smiles>C=C1CC[C@]2(C)[C@@H]3[C@@H](CC4=CC(=O)C=C[C@@]43O)[C@@]2(C)CC[C@@H]1C</smiles>

285

Figure 32. Structures of sesquiterpene-based meroterpenoids 278-285. 
Cinerols A-K (286-296) (Figure 33) were produced by the sponge Dysidea cinerea which was collected from the China Sea. Compounds 286-288 illustrated good PTP1B inhibitory effects with $\mathrm{IC}_{50}$ values of 3.8-8.8 $\mu \mathrm{M}$. On the other hand, only compound 291 was active towards the SHP-1 enzyme with $\mathrm{IC}_{50}$ : of $2.7 \mu \mathrm{M}$ [94]. Saccharoquinoline (297) was isolated from the bacterium Saccharomonospora sp. and featured a drimanetype sesquiterpene unit. Saccharoquinoline (286) exhibited good cytotoxicity towards HCT-116 cancer [95]. Three sesquiterpene based meroterpenes 298-300 were isolated from the sponge Dactylospongia elegans and compounds $\mathbf{3 0 0}$ illustrated cytotoxic effects towards SW1990, DU145, PANC-1, and Huh7 with $\mathrm{IC}_{50}$ values ranging from 2.3-37.8 $\mu \mathrm{M}$ [96]. Septosones A-C (301-303) were isolated from the sponge Dysidea septosa and septosone A (301) displayed good in vivo anti-inflammatory effects [97]. Terretonin N (304) (Figure 33) isolated from Nocardiopsis sp. illustrated a $15 \mathrm{~mm}$ of zone of inhibition towards Staphylococcus warneri, which has been observed to be even higher than the reference drug, gentamycin (14 mm) [98].

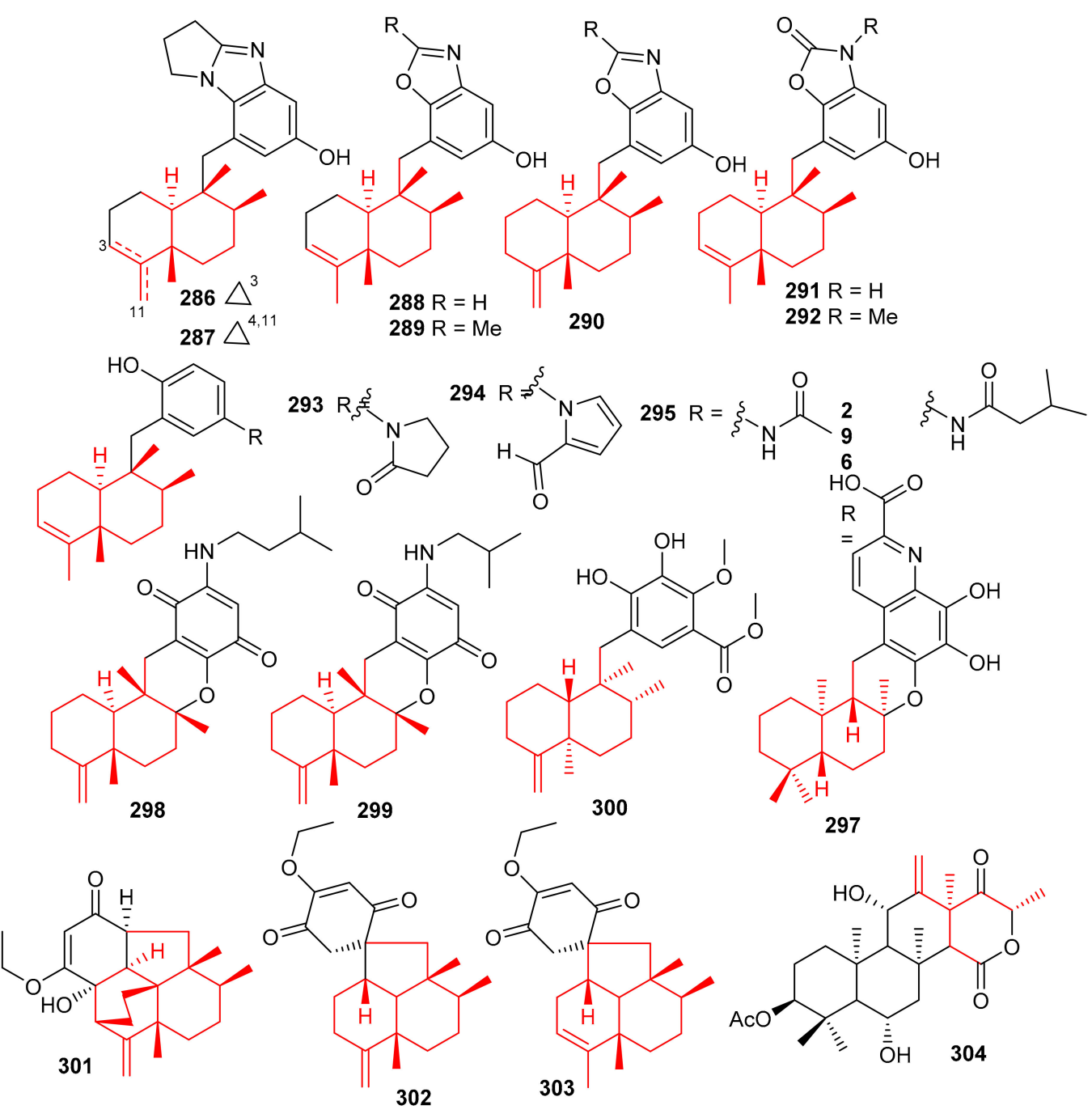

Figure 33. Structures of sesquiterpene-based meroterpenoids 286-304.

\section{Chromane/Chromene and Flavone Derived Meroterpenoids}

Among other metabolites, the chromene-derived meroterpenoid with an additional furan ring within a prenyl moiety, tuberatolide B (305) (Figure 34), was initially reported from Botryllus tuberatus [99] and later from Sargassum macrocarpum [100]. This diastereomeric meroterpenoid is reported to display anticancer activity since it inhibits lung cancers (H1299 and A549), breast cancers (MDA-MB-453, MDA-MB-231, and MCF7), colon cancers 
(CT26, HCT116, and SW620), cervical cancer (HeLa), and prostate cancers (DU145 and PC3) [100]. The mechanistic study revealed that compound 305 inhibits the growth of cancer cells by the production ROS in HCT116, A549, and MDA-MB-231, cells. It also increases DNA damage by the formation of $\gamma \mathrm{H} 2 \mathrm{AX}$ foci and or the phosphorylation of Chk2 and H2AX, which proteins are generally associated with DNA damage [100].

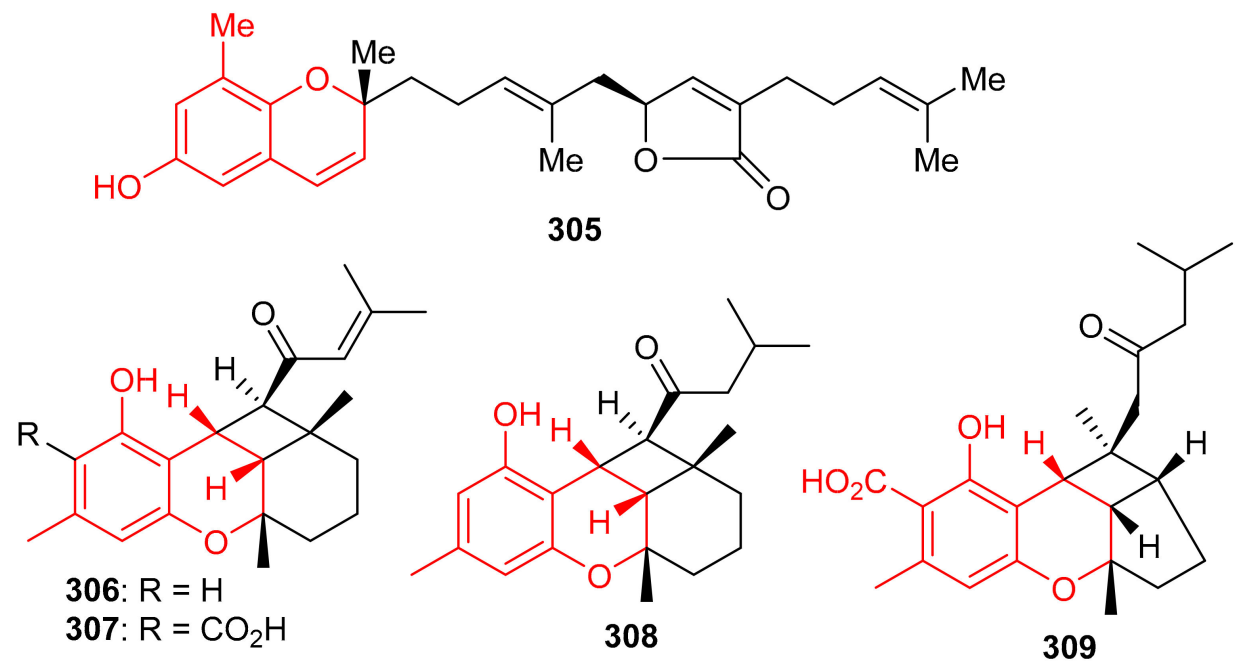

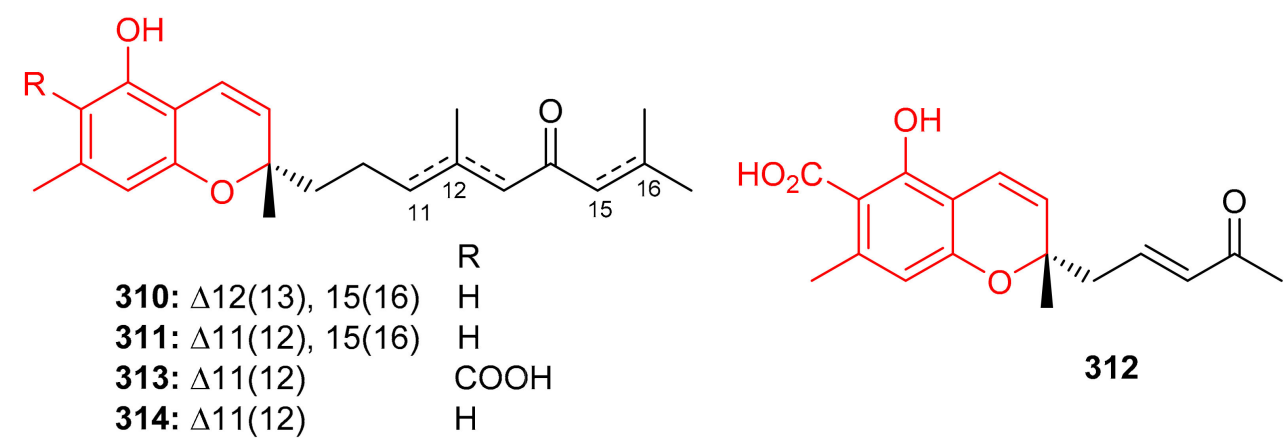

Figure 34. Structures of chromane/chromene derived meroterpenoids 305-314.

Chromane/chromene meroterpenoids (CMs), the rubiginosins A-G (306-312) (Figure 34) and anthopogochromenes A (313) and B (314), were reported from Rhododendron rubiginosum [101]. In addition to spectroscopic techniques, their absolute structures were established by making use of the chromane/chromene helicity rule, X-ray crystallography and $\mathrm{CD}$ analysis. All these compounds were evaluated for their cytotoxic effects towards A549, HCT116, SK-HEP-1, and HL-60 (Table 4). Compound 310 was the most active against all cell lines with $\mathrm{IC}_{50}$ values of $10.91,13.89,11.71$ and $7.40 \mu \mathrm{M}$, respectively and then followed compounds 306, 308 and 314. The other tested metabolites are reported to be inactive [101]. Doxorubicin ( $\mathrm{IC}_{50}=0.01-0.2 \mu \mathrm{M}$ ) was used as positive control in this study. Over $20 \mathrm{CMs}$ have been reported from the genus Rhododendron bearing a cannabinoid-like and orcinol core. Moreover, Rhododendron CM was also designated as a cannabicyclol (CBL)-type or cannabichromene (CBC)-type [102]. Interestingly CBC/CBL-type natural products having an orcinoid skeleton are rare in Cannabis and are mostly reported from Rhododendron species [102]. 
Table 4. Sesquiterpene-based, chromane/chromene and flavone derived meroterpenoids.

\begin{tabular}{|c|c|c|c|}
\hline Compounds & Source & Activities & Ref. \\
\hline Langcoquinone A (248) & Spongia sp. & $\begin{array}{c}\text { Antimicrobial effects: Staphylococcus aureus = MIC } 12.5 \\
\text { M; Bacillus subtilis }=\text { MIC } 12.5 \mu \mathrm{M}\end{array}$ & [82] \\
\hline Langcoquinone B (249) & Spongia sp. & $\begin{array}{c}\text { Antimicrobial effects: Staphylococcus aureus = MIC } 12.5 \\
\text { M; Bacillus subtilis = MIC } 12.5 \mu \mathrm{M}\end{array}$ & [82] \\
\hline Langconol A (250) & & Antimicrobial effects: B. subtilis MIC $12.5 \mu \mathrm{M}$ & [83] \\
\hline Langconol C (252) & & Antimicrobial effects: B. subtilis = MIC $25.0 \mu \mathrm{M}$ & [83] \\
\hline Aminoquinone (257) & Dysidea sp. & $\begin{array}{l}\text { Antimicrobial effects: } B . \text { subtilis = MIC } 50.0 \mu \mathrm{g} / \mathrm{mL} ; S \text {. } \\
\text { aureus = MIC } 50.0 \mu \mathrm{g} / \mathrm{mL} ; \text { E. coli }=\text { MIC } 50.0 \mu \mathrm{g} / \mathrm{mL}\end{array}$ & [84] \\
\hline Nakijinol G (267) & Hyrtios sp. & Enzyme Inhibition: $\mathrm{PTP} 1 \mathrm{~B}=\mathrm{IC}_{50} 4.8 \mu \mathrm{M}$ & [87] \\
\hline Dysivillosin A (270) & Dysidea villosa & Enzyme Inhibition: $\beta$-hexosaminidase $=\mathrm{IC}_{50} 8.2 \mu \mathrm{M}$ & [88] \\
\hline Dysivillosin B (271) & & Enzyme Inhibition: $\beta$-hexosaminidase $=\mathrm{IC}_{50} 10.2 \mu \mathrm{M}$ & [88] \\
\hline Dysivillosin C (272) & & Enzyme Inhibition: $\beta$-hexosaminidase $=\mathrm{IC}_{50} 19.9 \mu \mathrm{M}$ & [88] \\
\hline Chartarolide A (274) & Niphates recondite & $\begin{array}{l}\text { Cytotoxic effects: HCT-116 = IC } \mathrm{IC}_{50} 1.9 \mu \mathrm{M} ; \mathrm{HepG} 2= \\
\mathrm{IC}_{50} 1.8 \mu \mathrm{M} ; \mathrm{BGC}-823=\mathrm{IC}_{50} 1.3 \mu \mathrm{M} ; \mathrm{NCI}-\mathrm{H} 1650=\mathrm{IC}_{50} \\
5.5 \mu \mathrm{M} ; \mathrm{A} 2780=\mathrm{IC}_{50} 1.5 \mu \mathrm{M} ; \mathrm{MCF} 7=\mathrm{IC}_{50} 1.4 \mu \mathrm{M}\end{array}$ & {$[90]$} \\
\hline Chartarolide B (275) & Niphates recondite & $\begin{array}{l}\text { Cytotoxic effects: HCT-116 = IC } \mathrm{IC}_{50} 2.3 \mu \mathrm{M} ; \mathrm{HepG} 2= \\
\mathrm{IC}_{50} 2.8 \mu \mathrm{M} ; \mathrm{BGC}-823=\mathrm{IC}_{50} 1.6 \mu \mathrm{M} ; \mathrm{NCI}-\mathrm{H} 1650=\mathrm{IC}_{50} \\
4.8 \mu \mathrm{M} ; \mathrm{A} 2780=\mathrm{IC}_{50} 3.2 \mu \mathrm{M} ; \mathrm{MCF} 7=\mathrm{IC}_{50} 3.8 \mu \mathrm{M}\end{array}$ & [90] \\
\hline
\end{tabular}


Table 4. Cont

\begin{tabular}{|c|c|c|c|}
\hline Compounds & Source & Activities & Ref. \\
\hline Chartarolide C (276) & Niphates recondite & $\begin{array}{c}\text { Cytotoxic effects: HCT-116 = } \mathrm{IC}_{50} 7.8 \mu \mathrm{M} ; \\
\text { HepG2 = IC } \mathrm{I}_{50} 8.9 \mu \mathrm{M} ; \mathrm{BGC}-823=\mathrm{IC}_{50} 5.4 \mu \mathrm{M} ; \\
\text { NCI-H1650 }=\mathrm{IC}_{50} 11.3 \mu \mathrm{M} ; \mathrm{A} 2780=\mathrm{IC}_{50} 12.5 \\
\quad \mu \mathrm{M} ; \mathrm{MCF}=\mathrm{IC}_{50} 8.7 \mu \mathrm{M}\end{array}$ & {$[90]$} \\
\hline Terretonin N (304) & Nocardiopsis sp. & $\begin{array}{l}\text { Antimicrobial effects: } \text { S. warneri }=\mathrm{IZ} 14 \mathrm{~mm} \\
\qquad \text { E. coli }=\mathrm{IZ} 8 \mathrm{~mm}\end{array}$ & {$[98]$} \\
\hline Rubiginosin A (306) & Rhododendron rubiginosum & $\begin{array}{c}\text { Cytotoxic effects: } \mathrm{A} 549=\mathrm{IC}_{50} 16.15 \mu \mathrm{M} ; \\
\text { HCT116 }=\mathrm{IC}_{50} 15.56 \mu \mathrm{M} ; \mathrm{SK}-\mathrm{HEP}-1=\mathrm{IC}_{50} \\
13.80 \mu \mathrm{M} ; \mathrm{HL}-60=\mathrm{IC}_{50} 12.84 \mu \mathrm{M}\end{array}$ & [101] \\
\hline Rubiginosin B (307) & Rhododendron rubiginosum & $\begin{array}{c}\text { Cytotoxic effects: HCT116 = } \mathrm{IC}_{50} 65.72 \mu \mathrm{M} ; \\
\text { SK-HEP-1 }=\mathrm{IC}_{50} 84.66 \mu \mathrm{M}\end{array}$ & [101] \\
\hline Rubiginosin C (308) & Rhododendron rubiginosum & $\begin{array}{c}\text { Cytotoxic effects: } \mathrm{A} 549=\mathrm{IC}_{50} 40.45 \mu \mathrm{M} ; \\
\text { HCT116 }=\mathrm{IC}_{50} 17.43 \mu \mathrm{M} ; \mathrm{SK}-\mathrm{HEP}-1=\mathrm{IC}_{50} \\
26.26 \mu \mathrm{M} ; \mathrm{HL}-60=\mathrm{IC}_{50} 16.44 \mu \mathrm{M}\end{array}$ & [101] \\
\hline Rubiginosin D (309) & Rhododendron rubiginosum & $\begin{array}{c}\text { Cytotoxic effects: } \mathrm{A} 549=\mathrm{IC}_{50} 49.18 \mu \mathrm{M} ; \\
\text { HCT116 }=\mathrm{IC}_{50} 32.17 \mu \mathrm{M} ; \mathrm{SK}-\mathrm{HEP}-1=\mathrm{IC}_{50} \\
13.66 \mu \mathrm{M} ; \mathrm{HL}-60=\mathrm{IC}_{50} 40.07 \mu \mathrm{M}\end{array}$ & [101] \\
\hline Rubiginosin E (310) & Rhododendron rubiginosum & $\begin{array}{c}\text { Cytotoxic effects: } \mathrm{A} 549=\mathrm{IC}_{50} 38.90 \mu \mathrm{M} ; \\
\text { HCT116 = IC } \mathrm{IC}_{50} 38.90 \mu \mathrm{M} ; \mathrm{SK}-\mathrm{HEP}-1=\mathrm{IC}_{50} \\
38.90 \mu \mathrm{M} ; \mathrm{HL}-60=\mathrm{IC}_{50} 38.90 \mu \mathrm{M}\end{array}$ & [101] \\
\hline Rubiginosin F (311) & Rhododendron rubiginosum & $\begin{array}{c}\text { Cytotoxic effects: } \mathrm{A} 549=\mathrm{IC}_{50} 38.90 \mu \mathrm{M} ; \\
\text { HCT116 }=\mathrm{IC}_{50} 38.90 \mu \mathrm{M} ; \mathrm{SK}-\mathrm{HEP}-1=\mathrm{IC}_{50} \\
38.90 \mu \mathrm{M} ; \mathrm{HL}-60=\mathrm{IC}_{50} 38.90 \mu \mathrm{M}\end{array}$ & [101] \\
\hline Rubiginosins G (312) & Rhododendron rubiginosum & $\begin{array}{c}\text { Cytotoxic effects: } \mathrm{A} 549=\mathrm{IC}_{50} 38.90 \mu \mathrm{M} ; \\
\text { HCT116 = IC } \mathrm{IC}_{50} 38.90 \mu \mathrm{M} ; \mathrm{SK}-\mathrm{HEP}-1=\mathrm{IC}_{50} \\
38.90 \mu \mathrm{M} ; \mathrm{HL}-60=\mathrm{IC}_{50} 38.90 \mu \mathrm{M}\end{array}$ & [101] \\
\hline Anthopogochromene A (313) & Rhododendron rubiginosum & $\begin{array}{l}\text { Cytotoxic effects: } \mathrm{A} 549=\mathrm{IC}_{50} 38.90 \mu \mathrm{M} ; \\
\text { HCT116 = IC } 5038.90 \mu \mathrm{M} ; \mathrm{SK}-\mathrm{HEP}-1=\mathrm{IC}_{50} \\
38.90 \mu \mathrm{M} ; \mathrm{HL}-60=\mathrm{IC}_{50} 38.90 \mu \mathrm{M}\end{array}$ & [101] \\
\hline
\end{tabular}


Table 4. Cont.

\begin{tabular}{|c|c|c|c|}
\hline Compounds & Source & Activities & Ref. \\
\hline Anthopogochromene B (314) & Rhododendron rubiginosum & $\begin{array}{c}\text { Cytotoxic effects: A549 = IC } \mathrm{IC}_{50} 38.90 \mu \mathrm{M} ; \\
\text { HCT116 = IC }{ }_{50} 38.90 \mu \mathrm{M} ; \mathrm{SK}-\mathrm{HEP}-1=\mathrm{IC}_{50} \\
38.90 \mu \mathrm{M} ; \mathrm{HL}-60=\mathrm{IC}_{50} 38.90 \mu \mathrm{M}\end{array}$ & [101] \\
\hline Sargachromanol D (316) & Sargassum siliquastrum & $\begin{array}{c}\text { Antioxidant effects: DPPH }=\mathrm{EC}_{50} 26.35 \mu \mathrm{M} ; \\
\text { ABTS }=\mathrm{EC}_{50} 4.84 \mu \mathrm{M}\end{array}$ & [103] \\
\hline Sargachromanol G (318) & Sargassum siliquastrum & $\begin{array}{c}\text { Antioxidant effects: DPPH }=\mathrm{EC}_{50} 33.43 \mu \mathrm{M} ; \\
\text { ABTS }=\mathrm{EC}_{50} 4.05 \mu \mathrm{M}\end{array}$ & [103] \\
\hline Sargachromanol I (319) & Sargassum siliquastrum & $\begin{array}{c}\text { Antioxidant effects: DPPH }=\mathrm{EC}_{50} 32.83 \mu \mathrm{M} ; \\
\text { ABTS }=\mathrm{EC}_{50} 6.86 \mu \mathrm{M}\end{array}$ & [103] \\
\hline
\end{tabular}


Sargassum siliquastrum produced a small library of the meroterpenoids isopolycerasoidol (315), sargachromanols D (316), E (317), G (318), I (319), S (320), and T (321) (Figure 35) and all were evaluated for their antioxidant effects. Compound 315 was the most active in DPPH and ABTS antioxidant assays with $\mathrm{EC}_{50}$ values of 8.23 and $2.33 \mu \mathrm{M}$, respectively [103]. Compounds 316-318 were weakly active against the DPPH free radical, but induced significant inhibition (EC ${ }_{50}: 4.0$ to $4.8 \mu \mathrm{M}$, Table 4) against the ABTS free radical. On the other hand, compounds 316 and 317 are only weakly active in DPPH and ABTS antioxidant assays with $\mathrm{EC}_{50}$ values ranging from 15.7 to $57.0 \mu \mathrm{M}$. The structure and activity variation of compounds 316-318 suggest that the hydroxyl group at C-13 in the prenyl moiety can be the activity determining factor, since compounds 316 and 317 have the hydroxyl group at C-12, while compounds 318 and 319 have a corresponding keto function. Other literature results show that the chromene nucleus is an important group for antioxidant activities [103].

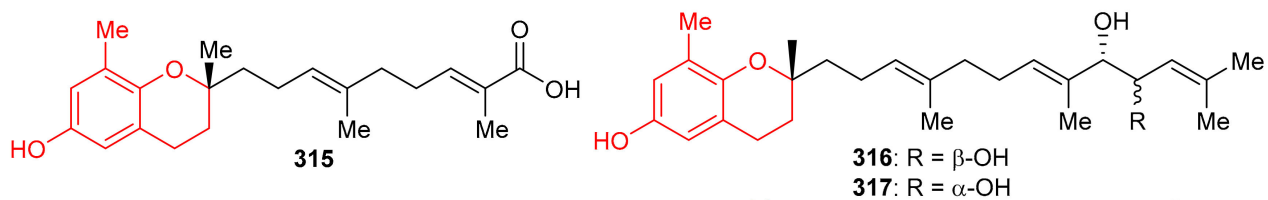<smiles>CC(C)=C[C@H](O)C(=O)C(C)CCC/C(C)=C/CC[C@]1(C)CCc2cc(O)cc(C)c2O1</smiles><smiles>CC(C)=CC(O)C(O)C(C)CCC/C(C)=C/CCC1(C)CCc2cc(O)cc(C)c2O1</smiles>

Figure 35. Structures of chromane/chromene derived meroterpenoids 315-321.

Another medicinal plant, Rhododendron capitatum produces enantiomeric pairs of meroterpenoids, the ( \pm )-rhodonoids C-G (322-326) (Figure 36). These compounds existed as racemates and were subsequently purified via chiral HPLC. Moreover, only 322a inhibited HSV-1 with an $\mathrm{IC}_{50}$ value of $80.6 \mu \mathrm{M}$. Compounds $322 \mathrm{a}$ and $\mathbf{3 2 2} \mathbf{b}$ featured the unusual $6 / 6 / 6 / 5$ tetracyclic ring core while compounds $323 \mathbf{a}$ and $\mathbf{3 2 3 b}$ bore the rather rare 6/6/5/5 tetracyclic ring system [104]. Another Rhododendron sp., viz., $R$. nyingchiense interestingly, also produced enantiomeric pairs of the meromonoterpenoids $\mathbf{3 2 7} \mathbf{a}, \mathbf{b}-\mathbf{3 3 2} \mathbf{a}, \mathbf{b}$ and all racemic mixtures were separated by chiral-phase HPLC. These compounds possess PTP1B inhibition with $\mathrm{IC}_{50}$ values ranging from 29 to $61 \mu \mathrm{M}$. Compounds $327 \mathbf{a}, \mathbf{b}$ feature a quite rare 6/6/5 tricyclic ring core [105].

Sargachromenol (333) (Figure 37) was produced by Sargassum serratifolium and inhibited $\mathrm{BChE}$ and $\mathrm{BACE} 1$ with values for $\mathrm{IC}_{50}: 9.4$ and $7.0 \mu \mathrm{M}$ respectively [106], while the reference compounds used were berberine $\left(\mathrm{IC}_{50}=9.4 \mu \mathrm{M}\right)$ and quercetin $\left(\mathrm{IC}_{50}=5.6\right.$ $\mu \mathrm{M})$ respectively. The alga Cystoseira baccata produced racemic mixtures of two meroterpenoids, tetraprenyltoluquinol $(\mathbf{3 3 4} \mathbf{a}, \mathbf{b})$, and tetraprenyltoluquinone $(\mathbf{3 3 5} \mathbf{a}, \mathbf{b})$. The in vitro anti-leishmanial study demonstrated that compound mixture $\mathbf{3 3 4} \mathbf{a}, \mathbf{b}$ exhibited reasonable effects towards Leishmania infantum with $\mathrm{IC}_{50}: 44.9 \mu \mathrm{M}$, whereas compound mixture 335a,b was found to be a weak inhibitor with $\mathrm{IC}_{50}$ of $94.4 \mu \mathrm{M}$. In an SAR study, it was determined that the C-1 ketone decreases the anti-leishmanial effects since the difference between these two compounds viz., 334 and 335 is the keto group [107]. 


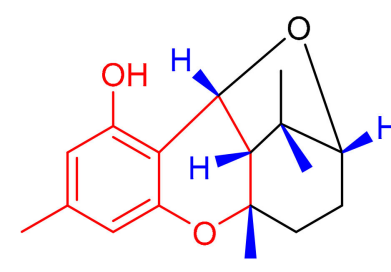

$322 a$

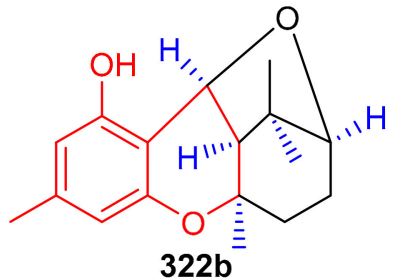

$322 \mathrm{~b}$<smiles>Cc1cc(O)c2c(c1)O[C@]1(C)CCC[C@H]3C(C)(C)O[C@H](O)[C@]231</smiles>

$323 a$<smiles>Cc1cc(O)c2c(c1)O[C@]1(C)CCC[C@@H]3[C@H]1[C@@H]2OC3(C)C</smiles>

$323 b$

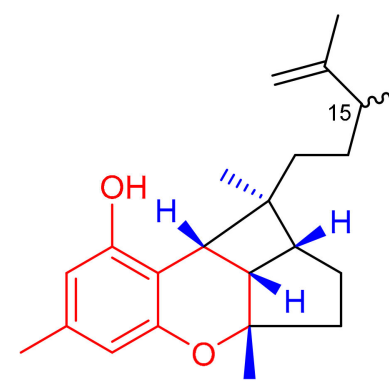

324a: $15 R$

325a: $15 S$

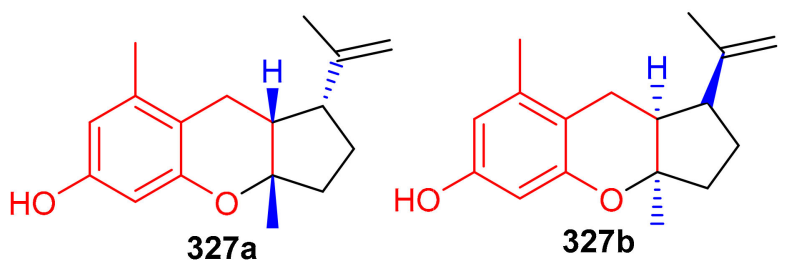

324b: $15 S$

325b: $15 R$<smiles>Cc1cc(O)c2c(c1)O[C@]1(C)CC[C@H](C(C)(C)O)[C@H]2C1</smiles>

$326 a$<smiles>Cc1cc(O)c2c(c1)O[C@]1(C)CC[C@H](C(C)(C)O)[C@H]2C1</smiles>

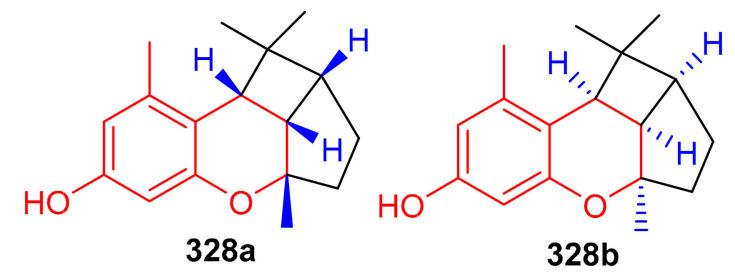

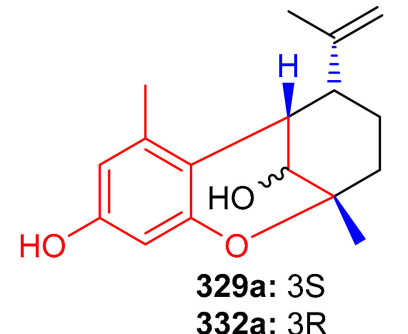<smiles>[R16][R16]1([H])Oc2cc(O)cc(C)c2[C@H]2[C@@H](C(=C)C)CCC(C)(C)[C@@H]21</smiles><smiles>[Y19][R](=O)[PH]([R])=O</smiles>

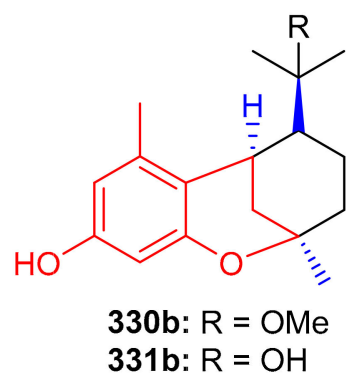

Figure 36. Structures of chromane/chromene derived meroterpenoids 322-332. 
<smiles>CC(C)=CCC/C(=C/CC/C(C)=C/CCC1(C)CCc2cc(O)cc(C)c2O1)C(=O)O</smiles><smiles>COc1cc(C)c2c(c1)CC[C@@](C)(CC1=C(CC(C)(C)O)C(=O)[C@@]3(C)CCC[C@]3(C)C1)O2</smiles><smiles>[R2]c1cc([R1])c2c(=O)cc([C@@]34C(=O)C=C(OC)C(=O)[C@@]3(C)CC=C(C)[C@@H]4C[C@]3(C)[C@@H](C(=O)O)CC=C4CCCC[C@H]43)oc2c1</smiles>

336: $\mathrm{R}_{1}=\mathrm{OH}, \mathrm{R}_{2}=\mathrm{OMe}$

337: $R_{1}=R_{2}=\mathrm{OH}$

338: $R_{1}=H, R_{2}=O M e$

339: $R_{1}=H, R_{2}=O H$<smiles>COc1cc(C)c2c(c1)C(=O)C[C@@](C)(CC1=C(CC(C)(C)O)C(=O)[C@@]3(C)CCC[C@]3(C)C1)O2</smiles>

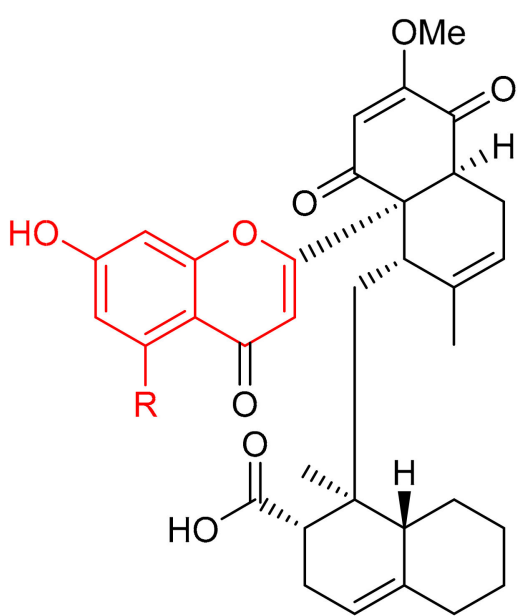

340: $\mathrm{R}=\mathrm{OH}$

341: $\mathrm{R}=\mathrm{H}$

Figure 37. Structures of chromane/chromene derived meroterpenoids 333-341.

Diplomeroterpenoids A-F (336-341) (Figure 37), were isolated from the roots of $\mathrm{Mi}$ mosa diplotricha and featured the diterpenoid unit and chromen-4-one framework. Compounds 336-338 and 340 inhibited protein farnesyl transferase (PFTase) with an $\mathrm{IC}_{50}$ ranging from 5.0 to $8.5 \mu \mathrm{M}$ [108]. Activity of the reference inhibitor FTase inhibitor II is reported as $\mathrm{IC}_{50}=0.1 \mu \mathrm{M}$. Additionally, diplomeroterpenoid A (336) is also reported to inhibit the growth of HepG2 cancer cells with a $\mathrm{GI}_{50}: 8.6 \mu \mathrm{M}$.

Glabralides C (342) was isolated from Sarcandra glabra [109]. Five pure meroterpenoid enantiomers (343a/343b-347a/347b) were produced by Rhododendron fastigiatum. Moreover, meroterpenoids 344a/344b, 345a/345b, demonstrated PTP1B inhibitory effects with $\mathrm{IC}_{50}$ ranging from 40.9 to 47.0 [110]. (+)-/(-)-Anthoponoids A-G, (348-354) (Figure 38), and $(+) /(-)$-daurichromene D (355) were isolated from Rhododendron anthopogonoides and (+)-anthoponoid E (352a), (-)-anthoponoid G (354b), exhibited potent anti-inflammatory effects in RAW 264.7 macrophages [111]. 


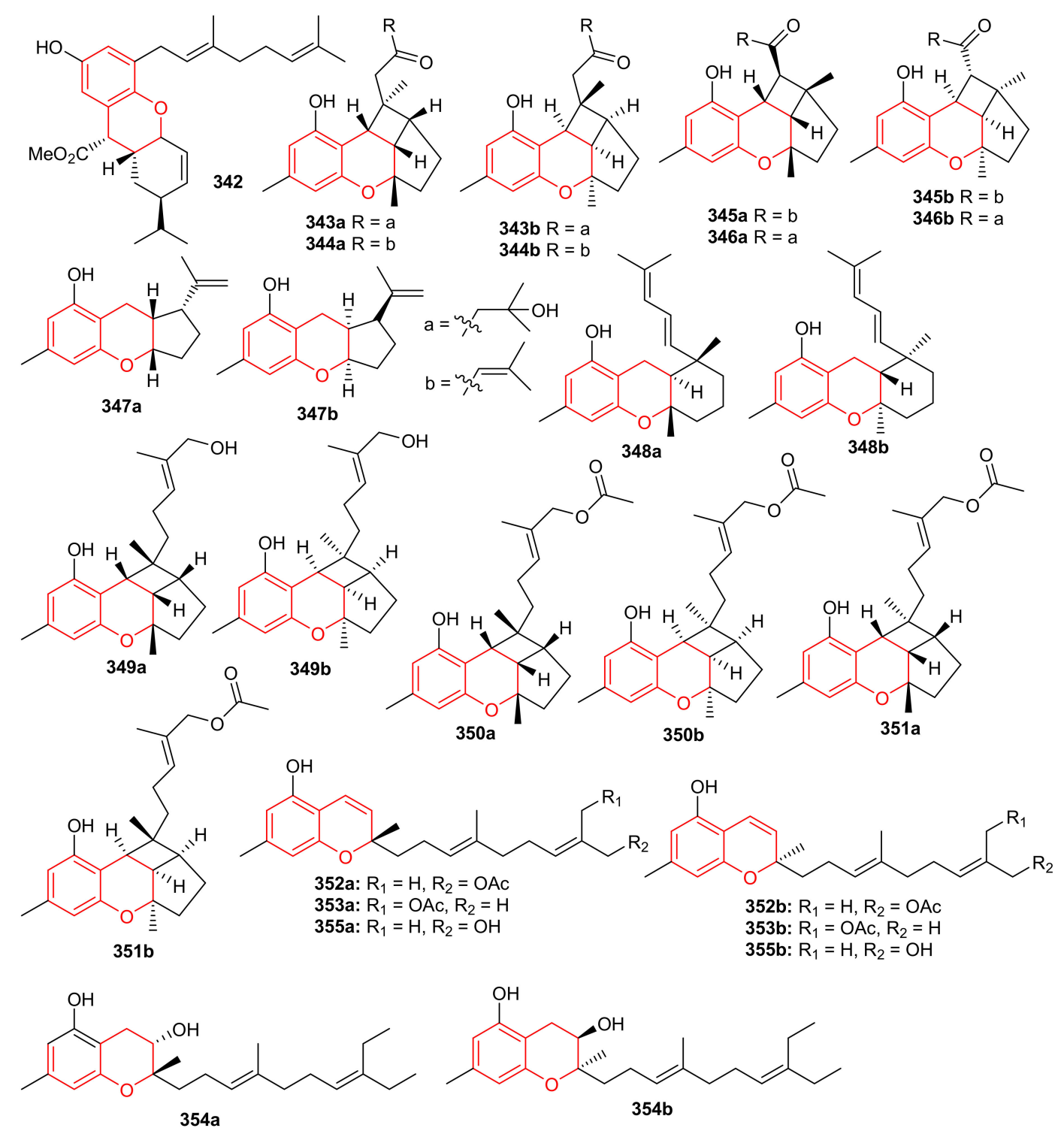

Figure 38. Structures of chromane/chromene derived meroterpenoids 342-354.

Psocorylins A-J (356-365) (Figure 39) were produced by Psoralea corylifolia and evaluated for their cytotoxic effects towards HepG2, NCI-N87, HeLa, HCT-116, and B16-F10. Meroterpene 357 displayed remarkable cytotoxic effects towards these five cell lines with $\mathrm{IC}_{50}:<10 \mu \mathrm{M}$. On the other hand, compounds 358-360 also illustrated significant effects towards HepG2 and NCI-N87 with $\mathrm{IC}_{50}$ : $<10 \mu \mathrm{M}$, which demonstrated selectivity towards these two cancer cells. Compound 356 illustrated no cytotoxicity but indicated the decreased cytotoxic effects due to the presence of a methoxy group at C-7. Notably, compound 361 displayed potent cytotoxic effects towards HepG2, NCI-N87, HeLa, and HCT-116 with $\mathrm{IC}_{50}$ values from 1.82 to $5.74 \mu \mathrm{M}$, respectively [112]. Belamcandanins A-C (366-368), were isolated from Belamcanda chinensis [113]. The highly functionalized, flavonoid-derived triterpene saponin meroterpenoids, clinoposides G (369) and H (370) (Figure 39) were isolated from Clinopodium chinense. Both compounds featured a triterpenoid core linked to a flavonoid framework via a C-C bond. The cardioprotective effects of compounds 369 and 370 were evaluated and both compounds illustrated protective effects towards anoxia/reoxygenation(A/R)-induced injury in H9c2 cells [114]. 


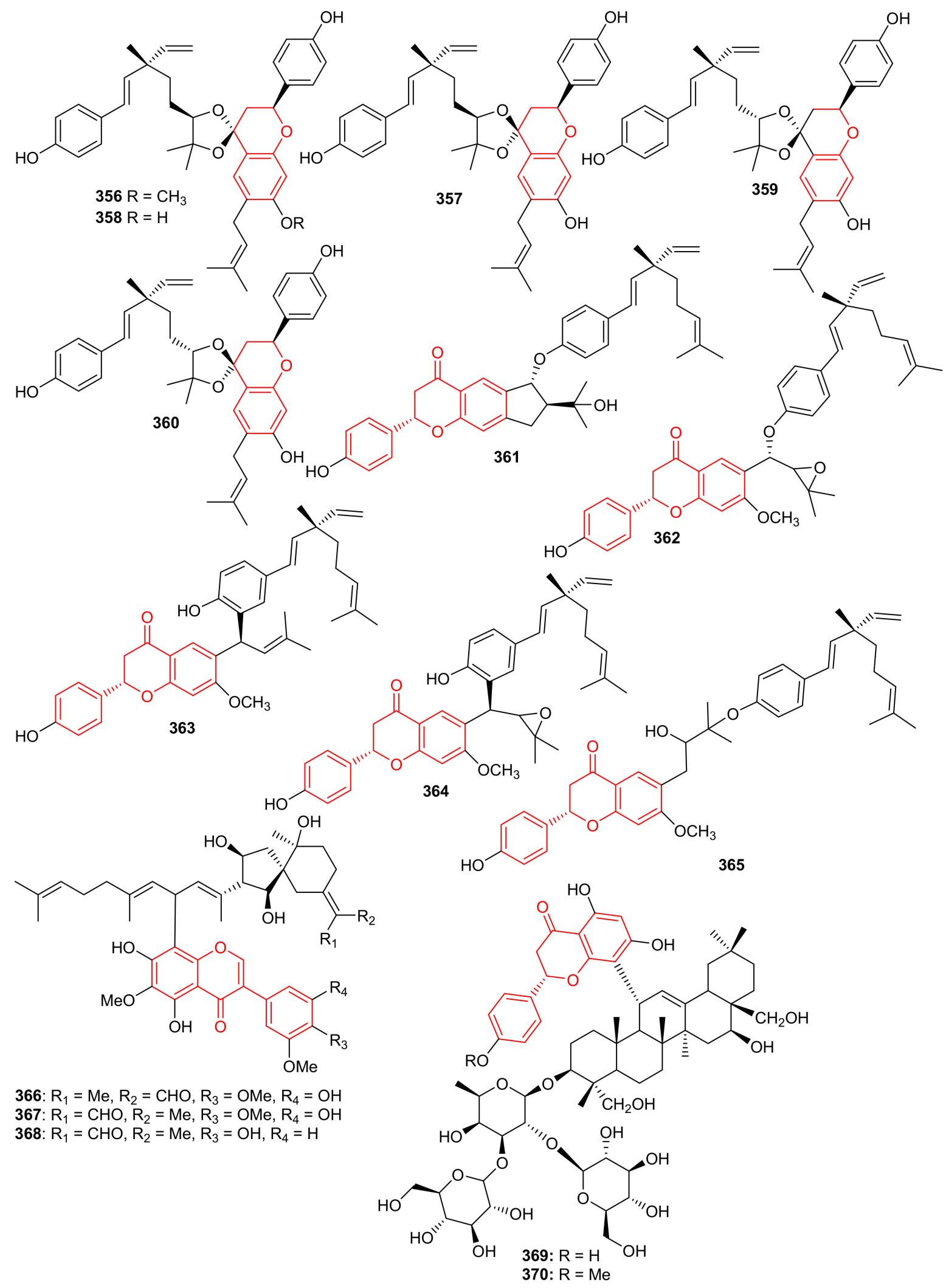

Figure 39. Structures of chromane/chromene and flavone derived meroterpenoids 356-370.

\section{Quinone-Based Meroterpenoids}

Streptomyces sp. produced naphthoquinone based meroterpenoids described as naphthablins B (371) and C (372) (Figure 40) and the absolute structures of these compounds were established by ECD spectra and the TDDFT approach. Compounds 371 and 372 were 
reported to be weakly active towards HeLa cells (19 to 32\%) [115]. Magterpenoid C (373) was reported from Magnolia officinalis var. and illustrated significant PTP1B inhibition with an $\mathrm{IC}_{50}$ value of $0.81 \mu \mathrm{M}$ [116].

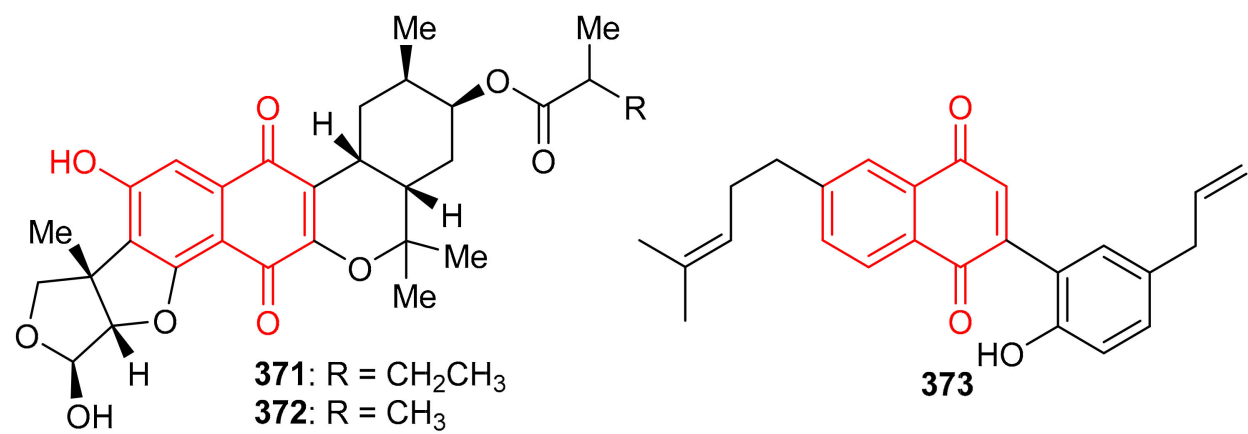

Figure 40. Structures of quinone-based meroterpenoids 371-373.

In 2018, arnebinone B (374), compound 375, arnebifuranone (376), and arnebinone (377) (Figure 41) were isolated from Arnebia euchroma and tested against various liver cancer cell lines viz., SMMC-7721, HepG2/ADM HepG2, and QGY-7703. Compound 375 was the most active among all the tested cell lines with $\mathrm{IC}_{50}$ values ranging from 3.43 to 11.31 $\mu \mathrm{M}$, while compound 374 had $\mathrm{IC}_{50}$ values ranging from 9.6 to $18.7 \mu \mathrm{M}$. These activites are reported even higher than the activity of the reference drug Cisplatin $\left(\mathrm{IC}_{50}=5.66-\right.$ $27.96 \mu \mathrm{M})$. On the other hand, the activities of compounds 376 and 377 were not that impressive (Table 5) [117]. Meroterpneoid 375 was also reported but with a different name viz., JNU-144, from Lithospermum erythrorhizon as a new compound in the same year (2018). The present study seems interesting in the sense that compound $\mathbf{3 7 5}$ has been reported to suppress cell viability and proliferation in hepatoma cells [118]. Toluquinol-derived meroterpenoid (378) bearing a tetraprenyl moiety, was isolated from the Carteriospongia sp. and was subsequently shown to trigger MMP disruption and apoptosis in lymphoma (U937 and Sup-T1 cells), leukemia (Molt 4 and HL60 cells), oral (Ca9-22 and Cal-27 cells), breast (T-47D cells) with $\mathrm{IC}_{50}$ values ranging from 0.33 to $1.06 \mu \mathrm{g} / \mathrm{mL}$ [119]. Doxorubicin, a positive control displayed $\mathrm{IC}_{50}$ values in the range of $0.1-2.47 \mu \mathrm{g} / \mathrm{mL}$, which revealed that the test compounds are equally active against the cell lines. Sargaquinoic acid (379) was reported from Sargassum serratifolium and inhibited the activity of AChE, BChE and BACE1, (Table 5) with $\mathrm{IC}_{50}$ values of $69.3,10.5$, and $12.1 \mu \mathrm{M}$ respectively [106].

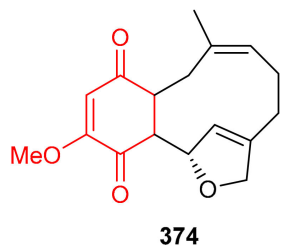

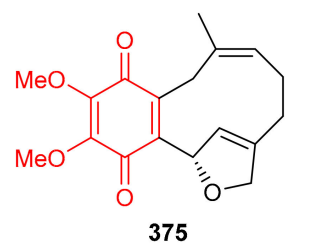<smiles>C=CC1(C)CCC(C(=C)C)C2=C1C(=O)C(OC)=C(OC)C2=O</smiles><smiles>CC(C)=CCC/C(=C\CC/C(C)=C/CC/C(C)=C/CC1=CC(=O)C=C(C)C1=O)CC/C(C)=C/CC/C(C)=C/CC/C(C)=C/CC/C(C)=C/CC1=CC(=O)C=CC1=O</smiles>

Figure 41. Structures of quinone-based meroterpenoids 374-379.

Two naphthoquinone based meroterpenes named Flaviogeranin B (380) and Flaviogeranin D (381) (Figure 42) were produced by Streptomyces sp. Notably, compound 381 illustrated significant antibacterial effects towards Mycobacterium smegmatis and Staphylococcus aureus with MIC values ranging from 5.2 to $9.2 \mu \mathrm{g} / \mathrm{mL}$. In addition, compound 381 also possessed potent cytotoxic effects towards HeLa and A549 with $\mathrm{IC}_{50}$ : 0.4 and $0.6 \mu \mathrm{M}$, respectively [120]. In 
another report Streptomyces sp. also produce merochlorins E (382) and F (383) which exhibited significant antibacterial effects towards B. subtilis, S. aureus, K. rhizophila with MIC ranging from $1-2 \mu \mathrm{g} / \mathrm{mL}[121]$.<smiles>COc1cc(O)c2c(c1N)C(=O)C(OCC=C(C)CCC=C(C)C)=C(C)C2=O</smiles><smiles>CC(C)=CCC/C(C)=C/CC1(O)CC(=O)c2c(cc(O)c(C)c2O)C1=O</smiles>

380<smiles>CC1=CCCC(C)(C)[C@H]1CC(C[C@@]1(O)C(=O)c2cc(O)cc(O)c2C(=O)C1(C)C)=C(C)C</smiles>

Figure 42. Structures of quinone-based meroterpenoids 380-383.

\section{Miscellaneous}

Sargahydroquinoic acid (384) (Figure 43) was isolated from Sargassum serratifolium and inhibited $\mathrm{BChE}$ and BACE1 with values of $\mathrm{IC}_{50}: 15.2$ and $4.4 \mu \mathrm{M}$ respectively [106]. Compared to the reference drugs berberine $\left(\mathrm{IC}_{50}=9.4 \mu \mathrm{M}\right)$ and quercetin $\left(\mathrm{IC}_{50}=5.6 \mu \mathrm{M}\right)$, respectively, the activities of these natural products are significant. Martucci et al. studied anticancer properties of tetronasin (385) which was obtained from Streptomyces sp. CP26-58 by HRLCMS. Tetronasin (385) killed the HeLa cells with an $\mathrm{IC}_{50}$ value of $0.23 \mu \mathrm{M}$ [115].<smiles>CC(C)=CCCC(=CCCC(C)=CCCC(C)=CCc1cc(O)cc(C)c1O)C(=O)O</smiles>

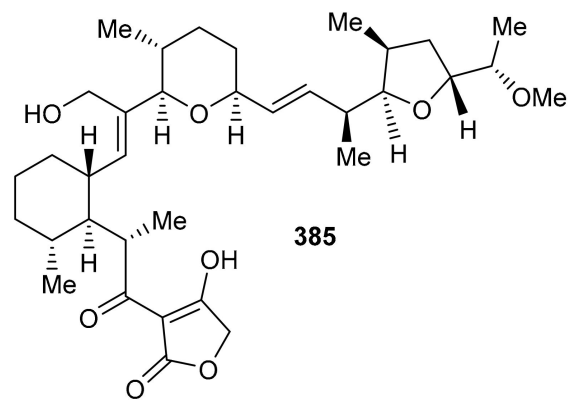

Figure 43. Structures of miscellaneous meroterpenoids 384 and 385.

Magterpenoids A (386) and B (387) (Figure 44) were purified from the bark extract of Magnolia officinalis and tested for PTP1B inhibitory activities. Compound 386 displayed a significant inhibition of the enzyme with an $\mathrm{IC}_{50}$ value of $1.44 \mu \mathrm{M}$, which is higher than the positive control drugs donepezil (45.3\% and 46.2\%). Moreover magterpenoid A (386) featured an interesting 4,6,11-trioxatricyclo[5.3.1.01,5]undecane skeleton while compound 387 had a 6/6/6/6 tetracyclic core [116]. Nyingchinoids A (388a,b) and B $(\mathbf{3 8 9} \mathbf{a}, \mathbf{b})$ were separated from the plant Rhododendron nyingchiense. Metabolites $\mathbf{3 8 8} \mathbf{a} / \mathbf{b}$ and $389 a, b$ featured $6 / 7 / 5 / 5$ and $6 / 6 / 3 / 5$ heterocyclic ring frameworks respectively. Notably, compounds $388 \mathbf{a} / \mathbf{b}$ and $\mathbf{3 8 9} \mathbf{a}, \mathbf{b}$ illustrated PTP1B effects with IC $_{50}$ values of 43.6 and 38.1 $\mu \mathrm{M}$, respectively. In this assay, oleanolic acid was used as positive control, which showed an $\mathrm{IC}_{50}$ value of $2.5 \mu \mathrm{M}$ [105]. 


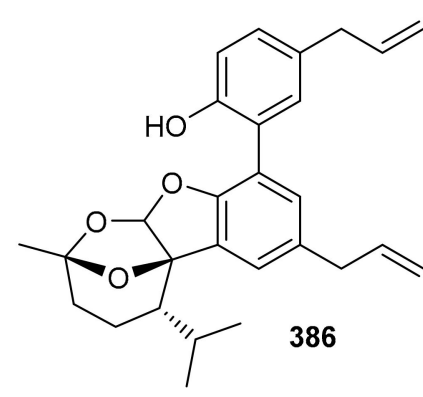<smiles>C=CCCC1=CCC23CC(=O)C=C[C@](O)(Oc4ccc(CC=C)cc42)C3C1</smiles><smiles>C=C(C)Cc1cc(C)c2c(c1)O[C@H]1[C@H](C)CC[C@H]1C(C)(C)O[C@H]2C</smiles><smiles>Cc1cc(O)cc2c1O[C@H]1OC(C)(C)[C@@H]3CC[C@H]([13CH2])[C@H]1[C@H]2O3</smiles>

(士)-387<smiles>CC1=CC(=O)C=C2O[C@]3(C)CC[C@H](C(C)(C)O)[C@H]3[C@H]12</smiles>

$389 a$<smiles>CC1=CC(=O)C=C2O[C@@H]1[C@@H]1[C@@H](C(C)(C)O)CC[C@@]1(C)O2</smiles>

Figure 44. Structures of miscellaneous meroterpenoids 386-389.

O-Spirocyclic ether analogs viz., 390 and 391 along with butanolide (392) (Figure 45) were obtained from Villorita cyprinoides, which is a traditional seafood in the coastal regions of the Arabian Sea. Meroterpenoid 391 illustrated activity towards COX-1 ( $\left.\mathrm{IC}_{50}: 0.86 \mathrm{mg} / \mathrm{mL}\right)$ and COX-2 ( $\mathrm{IC}_{50}: 0.65 \mathrm{mg} / \mathrm{mL}$ ) enzymes, followed by compound 390 (COX-1: $\mathrm{IC}_{50}: 0.94 \mathrm{mg} / \mathrm{mL}$; COX-2: $\mathrm{IC}_{50}: 0.70 \mathrm{mg} / \mathrm{mL}$ ) [122]. On the other hand, compound 392 was also found to be active towards COX-1 ( $\left.\mathrm{IC}_{50}: 0.91 \mathrm{mg} / \mathrm{mL}\right)$ but slightly less active than COX-2 ( $\mathrm{IC}_{50}: 0.74$ $\mathrm{mg} / \mathrm{mL}$ ) when compared to compound 390 and 391. Further biological studies revealed that compounds 390-392 demonstrated 5-LOX inhibitory effects $\left(\mathrm{IC}_{50}=0.77,0.75,0.80 \mathrm{mg} / \mathrm{mL}\right.$, respectively and their effects were higher than the standard ibuprofen $\left(\mathrm{IC}_{50}=0.96 \mathrm{mg} / \mathrm{mL}\right)$. In addition, these meroterpenoids illustrated more potent selectivity indices (SI: IC 50 : 1.231.34) than ibuprofen (SI: $\left.\mathrm{IC}_{50}: 0.63\right)$. Meroterpenoids $390\left(\mathrm{IC}_{50}: 0.59 \mathrm{mg} / \mathrm{mL}\right)$ and $391\left(\mathrm{IC}_{50}\right.$ : $0.54 \mathrm{mg} / \mathrm{mL}$ ) possess potent DPPH antioxidant effects which are higher than the standard $\alpha$-tocopherol $\left(\mathrm{IC}_{50}=0.65 \mathrm{mg} / \mathrm{mL}\right)$. Of note, compound 392 illustrated slightly less activity $\left(\mathrm{IC}_{50}=0.69 \mathrm{mg} / \mathrm{mL}\right)$ than the previous two compounds [122].<smiles>CCCCC(=O)OCC[C@H]1CC=C[C@H]2[C@H]1C(=O)O[C@H]2CO</smiles>

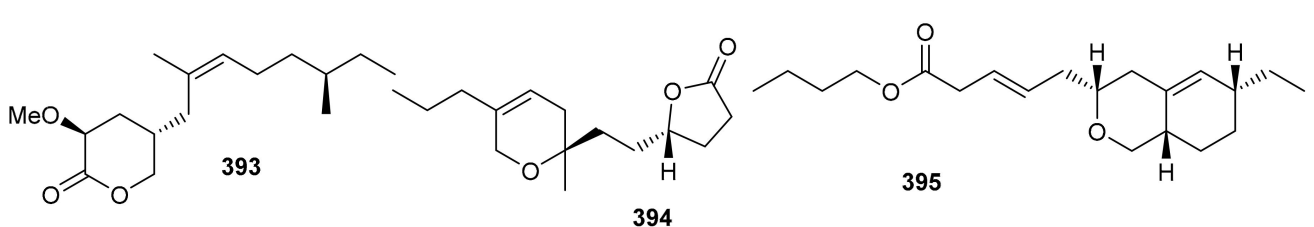<smiles>CCC/C=C/CC(=O)OC[C@H]1CC2=CCCC[C@H]2CO1</smiles>

Figure 45. Structures of miscellaneous meroterpenoids 390-396.

Villorita cyprinoides also produces four meroterpenoids viz., 393-396 (Figure 45) which were evaluated for various biological effects. Meroterpenoids 393-396 illustrated potent effects towards COX-1 and COX-2 in which the $\mathrm{IC}_{50}$ : ranged from 0.84 to $1.09 \mathrm{mg} / \mathrm{mL}$ [123], while the positive control ibuprofen exhibited $\mathrm{IC}_{50}$ values of 0.05 and $0.08 \mathrm{mg} / \mathrm{mL}$, respec- 
tively. Additionally, these compounds possess anti-COX-1/anti-COX-2 activity with $\mathrm{IC}_{50}$ : ranging from 1.12 to $1.22 \mathrm{mg} / \mathrm{mL}$ (Table 5). On the other hand, meroterpenoids 393-396 also display significant anti-5-LOX potential effects with $\mathrm{IC}_{50}$ values ranging from 0.76 to $0.98 \mathrm{mg} / \mathrm{mL}$ with the effects of $393\left(\mathrm{IC}_{50}=0.92 \mathrm{mg} / \mathrm{mL}\right)$ and $394\left(\mathrm{IC}_{50}=0.76 \mathrm{mg} / \mathrm{mL}\right)$ being higher than the standard ibuprofen $\left(\mathrm{IC}_{50}=0.96 \mathrm{mg} / \mathrm{mL}\right)$. Furthermore, meroterpenoids 393-396 also displayed DPPH antioxidant effects with $\mathrm{IC}_{50}$ values ranging from 0.63 to $0.79 \mathrm{mg} / \mathrm{mL}$ [123].

Erlotinib, the reference drug in this study, showed $\mathrm{IC}_{50}$ values of 4.5 and $7.66 \mu \mathrm{M}$, respectively. Meroterpenoids, 2-[tetrahydro-5-(4-hydroxyphenyl)-4-pentylfuran-3-yl]-ethyl-4-hydroxy benzoate (397), 2-2-[(4-hydroxybenzoyl)-oxy]-ethyl-4-methoxy-4-2-[(4-methylpentyl)oxy]-3,4dihydro-2H-6-pyranylbutanoic acid (398) and 3-[(5-butyl-3-methyl-5,6-dihydro-2H-pyran-2-yl)methyl]-4-methoxy-4-oxobutyl benzoate (399) (Figure 46) were reported from the alga Hypnea musciformis and were evaluated for their antioxidant effects. Compound 397 was more potent in terms of its DPPH radical effect $\left(\mathrm{IC}_{50}: 25.0 \mu \mathrm{M}\right)$ and this activity was higher than the standard gallic acid. On the other hand, compounds 398 ( $\left.\mathrm{IC}_{50}: 322.4 .0 \mu \mathrm{M}\right)$ and $399\left(\mathrm{IC}_{50}: 231.2 \mu \mathrm{M}\right)$ exhibit quite low antioxidant activity [124].<smiles>CCCCCC1C(CCOC(=O)c2ccc(O)cc2)COC1c1ccc(O)cc1</smiles>

Figure 46. Structures of miscellaneous meroterpenoids 397-399.

Cystoseira usneoides produces the meroterpenoids; cystodiones G-J (400-403), L (404) and M (405) along with cystones A-F (406-411) (Figure 47) [125]. All these compounds featured the toluhydroquinone core attached to a diterpenoid moiety. Compounds 400 411 illustrated radical-scavenging effects which ranged from $37 \%$ to $87 \%$. Among these compounds, cystodiones $\mathrm{G}(\mathbf{4 0 0} ; 81 \%)$ and $\mathrm{H}(401 ; 77 \%)$ were the most potent. Moreover, cystodione G (400) and cystodione M (405) significantly inhibited the TNF- $\alpha$ production with $81 \%$ and $79 \%$ respectively while cystone $C(408)$ demonstrated a moderate inhibition of 59\% [125]. 2-[(E)-Deca-1,8-dien-10-yl]-11,12-dihydro-13-propyl-2H-pyran (412) and $\left.1^{\prime}-[(10 E)-10-\{10-p e n t a n-4-y l\}-c y c l o h e x-4-e n y l]-a l l y l o x y\right)$-tetrahydro-2' ${ }^{\prime} 2^{\prime}$-dimethyl-2Hpyran (413) (Figure 47) were reported to be isolated from an animal source viz., Paphia malabarica [126]. In the DPPH antioxidant assay, compound 413 was slightly more potent $\left(\mathrm{IC}_{50}=0.76 \mathrm{mg} / \mathrm{mL}\right)$ than compound $412\left(\mathrm{IC}_{50}=0.78 \mathrm{mg} / \mathrm{mL}\right)$, while in the ABTS evaluation, compound $412\left(\mathrm{IC}_{50}=0.92 \mathrm{mg} / \mathrm{mL}\right)$ was slightly more active than compound $413\left(\mathrm{IC}_{50}=0.96 \mathrm{mg} / \mathrm{mL}\right)$. In addition both compounds were active towards COX-1 and COX-2 ( $\mathrm{IC}_{50}$ : 0.92 to $1.07 \mathrm{mg} / \mathrm{mL}$ ) along with selectivity indices of $\sim 1.1 \mathrm{mg} / \mathrm{mL}$. Moreover compounds $412\left(\mathrm{IC}_{50}=1.02 \mathrm{mg} / \mathrm{mL}\right)$ and $413\left(\mathrm{IC}_{50}=1.06 \mathrm{mg} / \mathrm{mL}\right)$ illustrated 5-LOX inhibition effects [126]. 
<smiles>[R7]Oc1c(C)cc(O)cc1C/C=C(\C)CC(=O)/C=C(\C)CC/C=C(\C)CCC(O)C(C)(C)C</smiles>

Figure 47. Structures of miscellaneous meroterpenoids 400-413.

The meroterpenoid enantiomers, $( \pm)$-rasumatranin A-D (414-417) and $( \pm)$-radulanin $\mathrm{M}$ (418) and $\mathrm{N}$ (419) along with meroterpenoids 420, 421 and ( \pm )-radulanin I (422) (Figure 48) were isolated from Radula sumatrana [127]. Compounds 414-417 and 421 (Figure 48) are monoterpene-bibenzyl hybrid metabolites while 421 and 422 are hemiterpenoid-bibenzyl hybrid compounds. Among these compounds, 421 proved to be very potent towards MCF-7 ( $\left.\mathrm{IC}_{50}: 3.8 \mu \mathrm{M}\right)$, PC-3 ( $\left.\mathrm{IC}_{50}: 6.6 \mu \mathrm{M}\right)$ and SMMC-7721 $7\left(\mathrm{IC}_{50}: 3.5 \mu \mathrm{M}\right)$ while 422 was only moderatively active towards these cancer cells with $\mathrm{IC}_{50}$ values $13.9-19.5 \mu \mathrm{M}$. On the other hand compounds $\mathbf{4 1 5}$ and $\mathbf{4 2 2}$ demonstrated moderate effects towards MCF-7 with $\mathrm{IC}_{50}: 38.3$ and $24.6 \mu \mathrm{M}$ respectively [127]. 
Table 5. Sources and biological effects of meroterpenoids 373-422.

\begin{tabular}{|c|c|c|c|}
\hline Compounds & Source & Activities & Ref. \\
\hline Magterpenoid C (373) & Magnolia officinalis & Enzyme Inhibition: PTP1B $=\mathrm{IC}_{50} 0.81 \mu \mathrm{M}$ & {$[116]$} \\
\hline Arnebinone B (374) & Arnebia euchroma & $\begin{array}{c}\text { Cytotoxic effects: HepG2, SMMC-7721, } \\
\text { QGY-7703 and HepG2/ADM IC } 50 \text { ranging } \\
\text { from } 9.6 \text { to } 18.7 \mu \mathrm{M}\end{array}$ & [117] \\
\hline Compound 375 & Arnebia euchroma & $\begin{array}{l}\text { Cytotoxic effects: HepG2, SMMC-7721, } \\
\text { QGY-7703 and HepG2/ADM IC } 50 \text { ranging } \\
\text { from } 3.43 \text { to } 11.31 \mu \mathrm{M}\end{array}$ & [117] \\
\hline Toluquinol-derivative (378) & Carteriospongia sp. & $\begin{array}{l}\text { Cytotoxic effects: Molt } 4=\mathrm{IC}_{50} 0.34 \mu \mathrm{g} / \mathrm{mL} ; \\
\mathrm{HL} 60=\mathrm{IC}_{50} 0.70 \mu \mathrm{g} / \mathrm{mL} ; \text { lymphoma U937 } \\
\mathrm{IC}_{50} 0.65 \mu \mathrm{g} / \mathrm{mL} ; \mathrm{Sup}-\mathrm{T} 1=\mathrm{IC}_{50} 0.33 \mu \mathrm{g} / \mathrm{mL} ; \\
\text { oral Ca9-22 = IC } 50.97 \mu \mathrm{g} / \mathrm{mL} ; \mathrm{Cal}-27=\mathrm{IC}_{50} \\
0.51 \mu \mathrm{g} / \mathrm{mL} ; \text { breast T-47D = } \mathrm{IC}_{50} 1.06 \mu \mathrm{g} / \mathrm{mL}\end{array}$ & [119] \\
\hline Sargaquinoic acid (379) & Sargassum serratifolium & $\begin{array}{c}\text { Enzyme Inhibition: } \mathrm{AChE}=\mathrm{IC}_{50} 69.3 \mu \mathrm{M} \\
\mathrm{BChE}=\mathrm{IC}_{50} 10.5 \mu \mathrm{M} ; \mathrm{BACE} 1=\mathrm{IC}_{50} 12.1 \mu \mathrm{M}\end{array}$ & [106] \\
\hline Sargahydroquinoic acid (384) & Sargassum serratifolium & $\begin{array}{l}\text { Enzyme Inhibition: } \mathrm{AChE}=\mathrm{IC}_{50} 124.3 \mu \mathrm{M} ; \\
\mathrm{BChE}=\mathrm{IC}_{50} 15.2 \mu \mathrm{M} ; \mathrm{BACE} 1=\mathrm{IC}_{50} 4.4 \mu \mathrm{M}\end{array}$ & [106] \\
\hline Magterpenoid A (386) & Magnolia officinalis & Enzyme Inhibition: PTP1B $=\mathrm{IC}_{50} 1.44 \mu \mathrm{M}$ & [116] \\
\hline Nyingchinoids A $(388 a, b)$ & Rhododendron nyingchiense & Enzyme Inhibition: PTP1B $=\mathrm{IC}_{50} 43.6 \mu \mathrm{M}$ & [105] \\
\hline Nyingchinoids B (389a,b) & Rhododendron nyingchiense & Enzyme Inhibition: PTP1B $=\mathrm{IC}_{50} 38.1 \mu \mathrm{M}$ & [105] \\
\hline Compound 390 & Villorita cyprinoides & $\begin{array}{c}\text { Antioxidant effects: } \mathrm{DPPH}=\mathrm{IC}_{50} 0.59 \\
\mathrm{mg} / \mathrm{mL} ; \mathrm{ABTS}=\mathrm{IC}_{50} 0.65 \mathrm{mg} / \mathrm{mL} ; \text { Enzyme } \\
\text { Inhibition: } \mathrm{COX}-1=\mathrm{IC}_{50} 0.94 \mathrm{mg} / \mathrm{mL} ; \\
\text { COX-2 }=\mathrm{IC}_{50} 0.70 \mathrm{mg} / \mathrm{mL}\end{array}$ & [122] \\
\hline Compound 391 & Villorita cyprinoides & $\begin{array}{c}\text { Antioxidant effects: } \mathrm{DPPH}=\mathrm{IC}_{50} 0.54 \\
\mathrm{mg} / \mathrm{mL} ; \mathrm{ABTS}=\mathrm{IC}_{50} 0.62 \mathrm{mg} / \mathrm{mL} ; \text { Enzyme } \\
\text { Inhibition: } \mathrm{COX}-1=\mathrm{IC}_{50} 0.86 \mathrm{mg} / \mathrm{mL} ; \\
\mathrm{COX}-2=\mathrm{IC}_{50} 0.65 \mathrm{mg} / \mathrm{mL}\end{array}$ & [122] \\
\hline
\end{tabular}


Table 5. Cont.

\begin{tabular}{|c|c|c|c|}
\hline Compounds & Source & Activities & Ref. \\
\hline Compound 392 & Villorita cyprinoides & $\begin{array}{c}\text { Antioxidant effects: } \mathrm{DPPH}=\mathrm{IC}_{50} 0.69 \\
\mathrm{mg} / \mathrm{mL} ; \mathrm{ABTS}=\mathrm{IC}_{50} 0.64 \mathrm{mg} / \mathrm{mL} ; \text { Enzyme } \\
\text { Inhibition: } \mathrm{COX}-1=\mathrm{IC}_{50} 0.91 \mathrm{mg} / \mathrm{mL} ; \\
\text { COX-2 }=\mathrm{IC}_{50} 0.74 \mathrm{mg} / \mathrm{mL}\end{array}$ & [122] \\
\hline $\begin{array}{l}\text { \{Tetrahydro-3-methoxy-5-((E)-8,12- } \\
\text { dimethyloct-8-enyl)-pyran-2-one } \\
(\mathbf{3 9 3})\end{array}$ & Villorita cyprinoides & $\begin{array}{c}\text { Antioxidant effects: } \mathrm{DPPH}=\mathrm{IC}_{50} 0.70 \\
\mathrm{mg} / \mathrm{mL} ; \mathrm{ABTS}=\mathrm{IC}_{50} 0.76 \mathrm{mg} / \mathrm{mL} ; \mathrm{Fe}^{2+}= \\
\mathrm{IC}_{50} 0.83 \mathrm{mg} / \mathrm{mL} ; \mathrm{H}_{2} \mathrm{O}_{2}=\mathrm{IC}_{50} 0.85 \mathrm{mg} / \mathrm{mL} ; \\
\mathrm{COX}_{-1}=\mathrm{IC}_{50} 0.99 \mathrm{mg} / \mathrm{mL} ; \mathrm{COX}-2=\mathrm{IC}_{50} 0.89 \\
\mathrm{mg} / \mathrm{mL}\end{array}$ & [123] \\
\hline $\begin{array}{c}\text { Dihydro-5-(8-(9,12-dihydro-8-methyl-11- } \\
\text { propyl-2H-pyran-8-yl)-ethyl)-furan-2(3H)- } \\
\text { one } \\
(\mathbf{3 9 4})\}\end{array}$ & Villorita cyprinoides & $\begin{array}{c}\text { Antioxidant effects: } \mathrm{DPPH}=\mathrm{IC}_{50} 0.63 \\
\mathrm{mg} / \mathrm{mL} ; \mathrm{ABTS}=\mathrm{IC}_{50} 0.79 \mathrm{mg} / \mathrm{mL} ; \mathrm{Fe}^{2+}= \\
\mathrm{IC}_{50} 0.83 \mathrm{mg} / \mathrm{mL} ; \mathrm{H}_{2} \mathrm{O}_{2}=\mathrm{IC}_{50} 0.84 \mathrm{mg} / \mathrm{mL} \\
\text { Enzyme Inhibition: } \mathrm{COX}-1=\mathrm{IC}_{50} 0.96 \\
\mathrm{mg} / \mathrm{mL} ; \mathrm{COX}-2=\mathrm{IC}_{50} 0.84 \mathrm{mg} / \mathrm{mL}\end{array}$ & [123] \\
\hline $\begin{array}{l}\text { Hexahydro-iso-chromenyl-meroterpenoid } \\
\qquad(395)\end{array}$ & Villorita cyprinoides & $\begin{array}{c}\text { Antioxidant effects: } \mathrm{DPPH}=\mathrm{IC}_{50} 0.76 \\
\mathrm{mg} / \mathrm{mL} ; \mathrm{ABTS}=\mathrm{IC}_{50} 0.82 \mathrm{mg} / \mathrm{mL} ; \mathrm{Fe}^{2+}= \\
\mathrm{IC}_{50} 0.90 \mathrm{mg} / \mathrm{mL} ; \mathrm{H}_{2} \mathrm{O}_{2}=\mathrm{IC}_{50} 0.86 \mathrm{mg} / \mathrm{mL} ; \\
\text { Enzyme Inhibition: } \mathrm{COX}-1=\mathrm{IC}_{50} 1.05 \\
\mathrm{mg} / \mathrm{mL} ; \mathrm{COX}-2=\mathrm{IC}_{50} 0.90 \mathrm{mg} / \mathrm{mL}\end{array}$ & [123] \\
\hline $\begin{array}{l}\text { Hexahydro-iso-chromenyl-meroterpenoid } \\
\qquad(396)\end{array}$ & Villorita cyprinoides & $\begin{array}{c}\text { Antioxidant effects: } \mathrm{DPPH}=\mathrm{IC}_{50} 0.79 \\
\mathrm{mg} / \mathrm{mL} ; \mathrm{ABTS}=\mathrm{IC}_{50} 0.81 \mathrm{mg} / \mathrm{mL} ; \mathrm{Fe}^{2+}= \\
\mathrm{IC}_{50} 0.89 \mathrm{mg} / \mathrm{mL} ; \mathrm{H}_{2} \mathrm{O}_{2}=\mathrm{IC}_{50} 0.87 \mathrm{mg} / \mathrm{mL} \\
\text { Enzyme Inhibition: } \mathrm{COX}-1=\mathrm{IC}_{50} 1.09 \\
\mathrm{mg} / \mathrm{mL} ; \mathrm{COX}-2=\mathrm{IC}_{50} 0.89 \mathrm{mg} / \mathrm{mL}\end{array}$ & [123] \\
\hline $\begin{array}{c}\text { 2-(Tetrahydro-5-(4-hydroxyphenyl)-4- } \\
\text { pentylfuran-3-yl)-ethyl-4-hydroxybenzoate } \\
\text { (397) }\end{array}$ & Hypnea musciformis & $\begin{array}{l}\text { Antioxidant effects: } \mathrm{DPPH}=\mathrm{IC}_{50} 25.05 \mu \mathrm{M} \\
\mathrm{Fe}^{2+} \text { ion chelating }=\mathrm{IC}_{50} 350.66 \mu \mathrm{M}\end{array}$ & [124] \\
\hline $\begin{array}{c}\text { 2-2-[(4-Hydroxybenzoyl)-oxy]-ethyl-4- } \\
\text { methoxy-4-2-[(4-methylpentyl)oxy]-3,4- } \\
\text { dihydro-2H-6-pyranylbutanoic acid } \\
(398)\end{array}$ & Hypnea musciformis & $\begin{array}{l}\text { Antioxidant effects: } \mathrm{DPPH}=\mathrm{IC}_{50} 322.4 \mu \mathrm{M} \\
\qquad \mathrm{Fe}^{2+} \text { ion chelating }=\mathrm{IC}_{50} 5115.3 \mu \mathrm{M}\end{array}$ & [124] \\
\hline
\end{tabular}


Table 5. Cont

\begin{tabular}{|c|c|c|c|}
\hline Compounds & Source & Activities & Ref. \\
\hline $\begin{array}{c}\text { 3-((5-Butyl-3-methyl-5,6-dihydro-2H-pyran-2- } \\
\text { yl)-methyl)-4-meth oxy-4-oxobutyl benzoate } \\
\text { (399) }\end{array}$ & Hypnea musciformis & $\begin{array}{l}\text { Antioxidant effects: } \mathrm{DPPH}=\mathrm{IC}_{50} 231.2 \mu \mathrm{M} \\
\mathrm{Fe}^{2+} \text { ion chelating }=\mathrm{IC}_{50} 667.9 \mu \mathrm{M}\end{array}$ & [124] \\
\hline $\begin{array}{c}\text { 2-((E)-deca-1,8-dien-10-yl)-11,12-dihydro-13- } \\
\text { propyl-2H-pyran } \\
(\mathbf{4 1 2})\end{array}$ & Paphia malabarica & $\begin{array}{c}\text { Antioxidant effects: } \mathrm{DPPH}=\mathrm{IC}_{50} 0.78 \\
\mathrm{mg} / \mathrm{mL} ; \mathrm{ABTS}=\mathrm{IC}_{50} 0.92 \mathrm{mg} / \mathrm{mL} ; \text { Enzyme } \\
\text { Inhibition: } \mathrm{COX}-1=\mathrm{IC}_{50} 1.07 \mathrm{mg} / \mathrm{mL} ; \\
\text { COX-2 = } \mathrm{IC}_{50} 0.95 \mathrm{mg} / \mathrm{mL} ; 5-\mathrm{LOX}=\mathrm{IC}_{50} 1.02 \\
\mathrm{mg} / \mathrm{mL}\end{array}$ & [126] \\
\hline $\begin{array}{c}1^{\prime}-((10 E)-10-(10-(\text { pentan-4-yl)-cyclohex-4- } \\
\text { enyl)-allyloxy)-tetrahydro-2', } \\
2^{\prime} \text {-dimethyl-2H-pyran (413) }\end{array}$ & Paphia malabarica & $\begin{array}{c}\text { Antioxidant effects: } \mathrm{DPPH}=\mathrm{IC}_{50} 0.76 \\
\mathrm{mg} / \mathrm{mL} ; \mathrm{ABTS}=\mathrm{IC}_{50} 0.96 \mathrm{mg} / \mathrm{mL} ; \text { Enzyme } \\
\text { Inhibition: } \mathrm{COX}-1=\mathrm{IC}_{50} 1.05 \mathrm{mg} / \mathrm{mL} ; \\
\mathrm{COX}-2=\mathrm{IC}_{50} 0.92 \mathrm{mg} / \mathrm{mL} ; 5-\mathrm{LOX}=\mathrm{IC}_{50} 1.06 \\
\mathrm{mg} / \mathrm{mL}\end{array}$ & [126] \\
\hline$( \pm)$-Rasumatranin B (415) & Radula sumatrana & Cytotoxic effects: MCF-7 $=\mathrm{IC}_{50}: 38.3 \mu \mathrm{M}$ & [127] \\
\hline Compound 421 & Radula sumatrana & $\begin{array}{l}\text { Cytotoxic effects: MCF-7: } \mathrm{IC}_{50}: 3.8 \mu \mathrm{M} \text {; PC-3: } \\
\mathrm{IC}_{50}: 6.6 \mu \mathrm{M} ; \mathrm{SMMC}-7721 \text { 7: } \mathrm{IC}_{50}: 3.5 \mu \mathrm{M}\end{array}$ & [127] \\
\hline
\end{tabular}




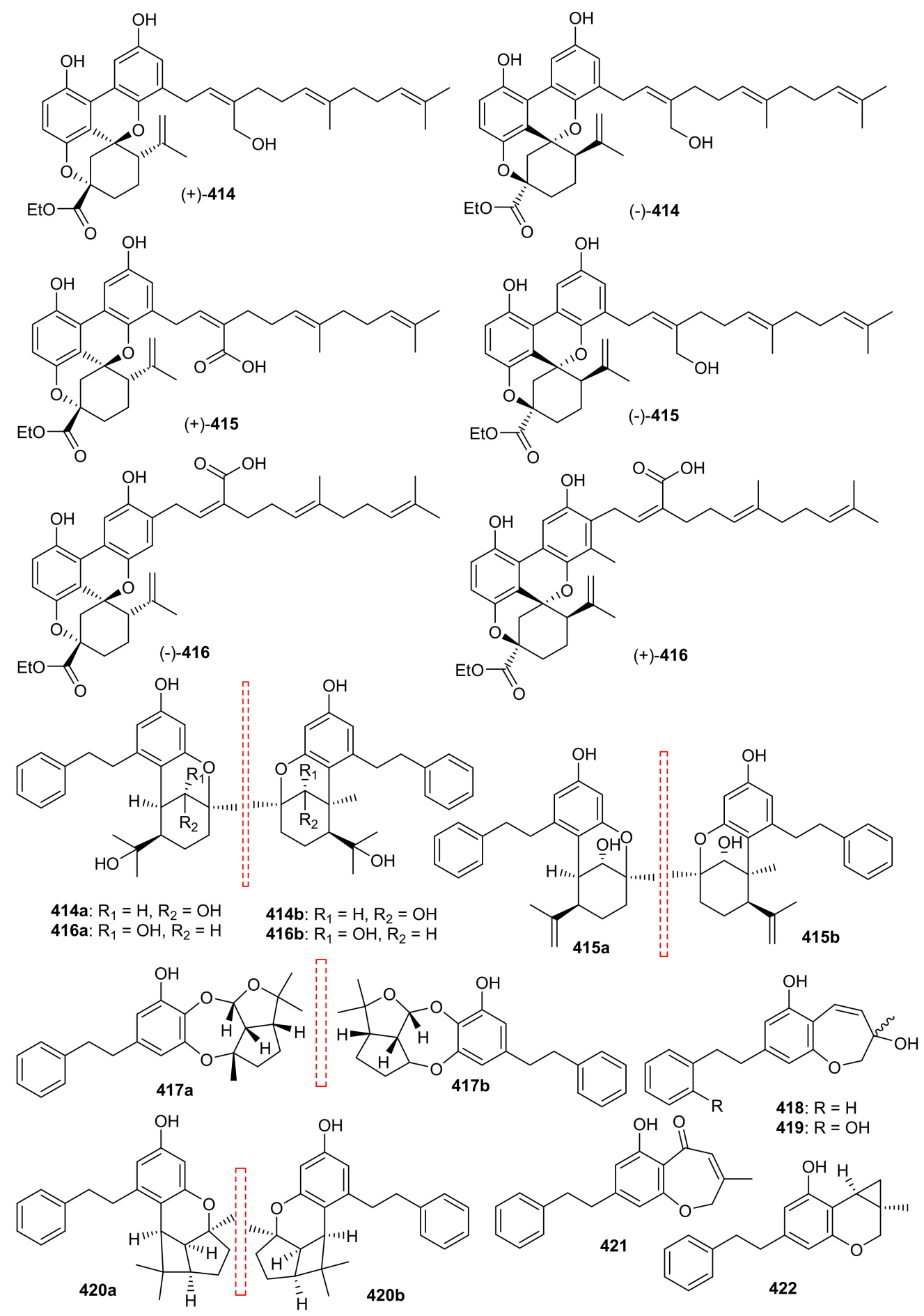

Figure 48. Structures of miscellaneous meroterpenoids 414-422.

Meroindenon (423) was isolated from Streptomyces and illustrated moderate antimicrobial effects towards B. subtilis, K. rhizophila, and S. aureus [121]. Hyperinoids A (424) and B (425) (Figure 49) were isolated from Hypericum patulum and both compounds significantly inhibited NF-kB in RAW 246.7 macrophages [128]. Meroterpenoids 426 and 427 were produced by the mushroom Panus lecomtei [129] and prenylbruceol A was isolated from Philotheca myoporoides [130]. Moreover 6-deoxytolypodiol (428) and 11-hydroxytolypodiol (429) were produced by cyanobacterial sources. Only compound 430 displayed potent antiinflammatory effects with $\mathrm{IC}_{50}: 0.1 \mu \mathrm{M}$ while the same compound possessed similar levels of $\mathrm{TXB}_{2}$ inhibitory effects as NSAID flurbiprofen [131]. Hyperprins A (431) and B (432) 
were produced by Hypericum przewalskii and both compounds featured a 6/6/6/6/5/5 hexacyclic core and a 6/8/6/6 tetracyclic system respectively [132]. In another report hypulatones A (433) and B (434) (Figure 49) were produced by Hypericum patulum and the latter compound remarkably inhibited the late current of $\mathrm{Na}_{\mathrm{v}} 1.5$ with $\mathrm{IC}_{50}: 0.2 \mu \mathrm{M}$ ) [133].

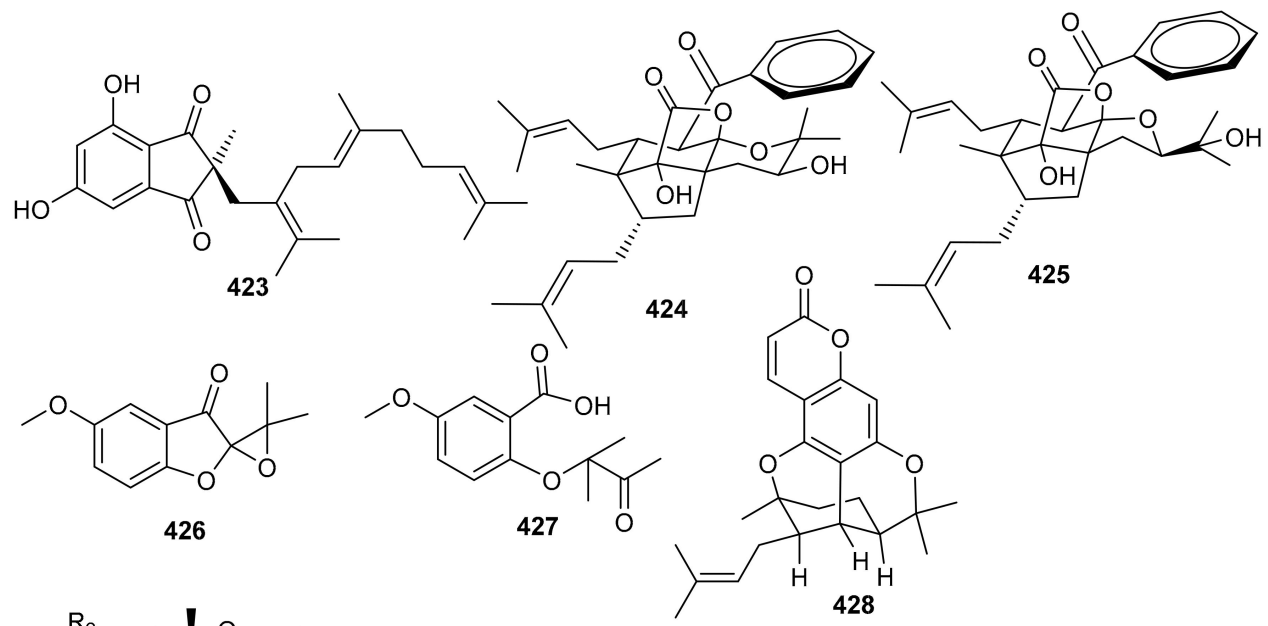

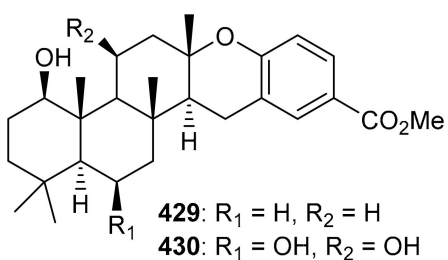

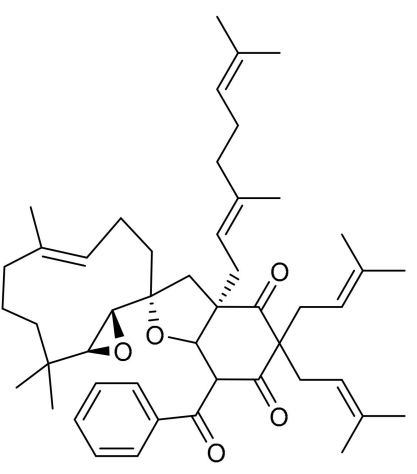

$( \pm)-433$

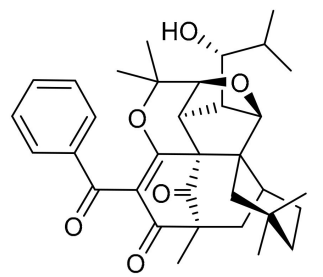

431

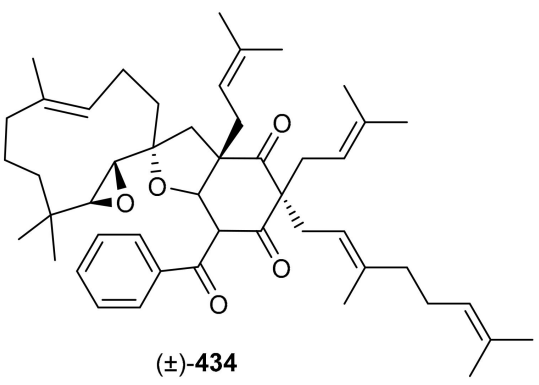

Figure 49. Structures of miscellaneous meroterpenoids 423-434.

Ampechromonol A (435) and B (436) (Figure 50) were produced by Ampelopsis cantoniensis and displayed weak cytotoxic effects towards MCF-7 cells [134]. Psoralea corylifolia seed produced $7 \beta, 13 \beta$-psoracorylifol B (437) and $7 \beta, 8 \alpha$-psoracorylifol D (438). Moreover, compounds $435-439$ displayed moderate inhibitory effects towards DGAT1 with IC $_{50}$ : 67.1 and $99.5 \mu \mathrm{M}$, respectively. On the other hand, both compounds 435 and 436 illustrated weak inhibition towards DGAT2 with $\mathrm{IC}_{50}: 132.9$ and $134.2 \mu \mathrm{M}$, respectively [135]. In another report, corypsoriols A-N (439-452) (Figure 50) were isolated from Psoralea corylifolia [136]. 
<smiles>[R]OC(=O)CCc1oc2cc(O)cc(O)c2c(=O)c1O</smiles>

435: $\mathrm{R}=\mathrm{H}$

436: $\mathrm{R}=\mathrm{Me}$<smiles>C=C[C@H]1CC[C@@H](C(=C)C)O[C@]1(C)[C@H](O)c1ccc(O)cc1</smiles><smiles>C=C[C@]1(C)CC[C@@H](C(C)C)[C@](C)(Cc2ccc(O)cc2)O1</smiles>

437<smiles>C=C[C@]1(C)CC[C@H](C(C)C)[C@@]2(C)C[C@H]1C[C@H]2c1ccc(O)cc1</smiles>

438<smiles>C=C[C@]1(C)CC[C@@H]2[C@@H](c3ccc(O)cc3C(C)(C)[C@@H]2O)[C@H]1O</smiles>

$4398-\mathrm{OH}, \beta$<smiles>[R][R14]#[R]</smiles>

$442 \mathrm{R}_{1}=\mathrm{H}, \mathrm{R}_{2}=\mathrm{A}$ $444 \mathrm{R}_{1}=\mathrm{OH}, \mathrm{R}_{2}=\mathrm{B}$

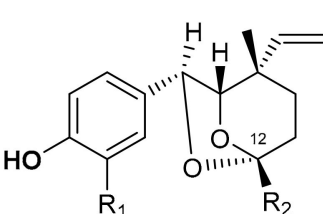

$443 \mathrm{R}_{1}=\mathrm{H}, \mathrm{R}_{2}=\mathrm{A}$ $445 \mathrm{R}_{1}=\mathrm{OH}, \mathrm{R}_{2}=\mathrm{B}$

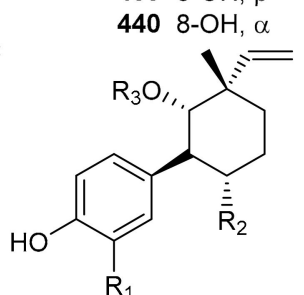

$\mathrm{R}_{1}$<smiles>C=C[C@]1(C)CC[C@@H](C(=C)C)[C@H](c2ccc(O)c(O)c2)[C@@H]1OC</smiles>

449<smiles>C=C[C@]1(C)CC[C@@H](C(=C)C)[C@H](c2ccc(O)c(O)c2)[C@@H]1OC</smiles>

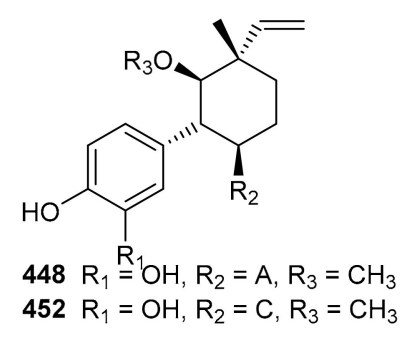

Figure 50. Structures of miscellaneous meroterpenoids 435-452.

\section{Conclusions}

In this review, the structures, chemical diversity, and biological properties of 452 new meroterpenoids have been reported. The chemical structures of meroterpenoids are extremely diverse, as may be noted by the various biosynthetic pathways and clearly demonstrated nature's sophisticated synthetic protocols to generate this tremendous chemical diversity via simple and achiral starting units. As comprehensively explored in each section, these types of secondary metabolites possess a tremendous structural diversity resulting from such reactions as condensation, alkylation, oxidation, and reduction. Moreover, meroterpenoids incorporated multiple prenyl moieties or very complex ring cores, which furnish abundant molecular scaffolds for such a wide range of biological activities.

Due to their structural diversity, meroterpenoids illustrated a wide range of biological and pharmacological activities, viz., antimicrobial, anticancer/cytotoxic, antioxidant, anti-inflammatory, antiviral immunosuppressive, and anti-Leishmania. Moreover, these compounds are also reported to possess important enzyme inhibitory effects, viz., acetylcholinesterase, protein tyrosine phosphatase 1B (PTP1B), BACE1, dehydrogenase 1 (IDH1), $\alpha$-glucosidase, influenza neuraminidase, Janus Kinase 3 (JAK3), HMG-CoA, aldose reductase, maltase, and $\beta$-hexosaminidase. Among phloroglucinol-derived metroterpenoids, psiguajavadial B (2), which was isolated from Psidium guajava, illustrated potent cytotoxic effects towards lung cancer (A549) with $\mathrm{IC}_{50} 0.90 \mu \mathrm{M}$. Moreover, eugenial C (18), which was produced by Eugenia umbelliflora, possessed significant cytotoxic effects towards leukemia cells (K562) with $\mathrm{IC}_{50} 0.38 \mu \mathrm{M}$. On the other hand, Eucalyptus robusta produced eucarobustols A (63), G (69), and I (71) and these compounds illustrated significant PTP1B inhibition (antidiabetic effects) with $\mathrm{IC}_{50} 1.3,1.8$, and $1.6 \mu \mathrm{M}$, respectively. Among syncarpic acid/ $\beta$ triketones-based meroterpenes, frutescones $\mathrm{O}(\mathbf{1 8 2})$ exhibited anti-inflammatory activity with an $\mathrm{IC}_{50}$ value of $0.36 \mu \mathrm{M}$ while hyperjaponols $\mathrm{B}$ (195a) D (197) demonstrated potent antiviral effects towards the EBV virus with $\mathrm{EC}_{50} 0.57$ and $0.49 \mu \mathrm{M}$ respectively.

Marinocyanin A (240) is an alkaloidal based meroterpenoid and is isolated from Actinomycete strains; it demonstrates potent cytotoxic effects towards colon cancer (HCT-116) with $\mathrm{IC}_{50} 0.049 \mu \mathrm{M}$. Furthermore, this compound also possesses significant antimicrobial 
effects towards Candida albicans (MIC: $0.95 \mu \mathrm{M}$ ), and Staphylococcus aureus (MIC: $2.3 \mu \mathrm{M}$ ). Similarly, another alkaloidal based meroterpenoid marinocyanin B (241) also demonstrated potent cytotoxic effects towards HCT-116 with $\mathrm{IC}_{50} 0.029 \mu \mathrm{M}$. Among sesquiterpene-based meroterpenoids, chartarolide A (274) was produced by Niphates recondite and demonstrated significant cytotoxic effects towards HCT-116, HepG2, BGC-823, A2780, and MCF7 with $\mathrm{IC}_{50} 1.9,1.8,1.3,1.5$, and $1.4 \mu \mathrm{M}$, respectively. Magterpenoid C (373) demonstrated potent antidiabetic effects $\left(\mathrm{PTP} 1 \mathrm{~B}=\mathrm{IC}_{50} 0.81 \mu \mathrm{M}\right)$. Notably meroterpene 378 possessed potent cytotoxic effects towards Molt 4, HL60, lymphoma U937, Sup-T1, oral Ca9-22, and with $\mathrm{IC}_{50}<1.0 \mu \mathrm{g} / \mathrm{mL}$. In addition, tetronasin (385) which was produced by Streptomyces sp., illustrated significant cytotoxic effects towards HeLa cells with $\mathrm{IC}_{50} 0.23 \mu \mathrm{M}$. From the large library of bioactive meroterpenoids described in this detailed review, we hope a reasonable range of new lead structures may enter into the drug development process to treat debillitating diseases in the future.

Author Contributions: All authors made contributions to preparing this manuscript. Conceptualization, M.N., M.S. and H.H.; writing—original draft preparation, M.N., M.S., M.I.T., M.A.A., F.S., I.A. (Iftikhar Ali), N.Z.M. and I.A. (Ishtiaq Ahmed); writing-review and editing, D.W., I.R.G., H.H., M.L.A. and M.S.; funding acquisition, E. and M.L.A. All authors have read and agreed to the published version of the manuscript.

Funding: This research was funded by the Deanship of Scientific Research at Princess Nourah Bint Abdulrahman University through the Fast-track Research Funding Program.

Acknowledgments: The authors (H.H. and N.Z.M.) thanks the Alexander von Humboldt Foundation for its generous support in providing the opportunity to work in Germany which facilitated the writing of this review. The authors extend their appreciation to the Deanship of Scientific Research at Princess Nourah Bint Abdulrahman University for funding this work through the Fast-track Research Funding Program.

Conflicts of Interest: The authors declare no conflict of interest.

\section{References}

1. Brahmachari, G. Discovery and Development of Neuroprotective Agents from Natural Products: An Overview. In Discovery and Development of Neuroprotective Agents from Natural Products, 1st ed.; Brahmachari, G., Ed.; Elsevier: Amsterdam, The Netherlands, 2018; pp. 1-7.

2. Swargiary, A. Recent trends in traditionally used medicinal plants and drug discovery. Asian J. Pharm. Pharmacol. 2017, 3, 111-120.

3. Annang, F.; Genilloud, O.; Vicente, F. Contribution of Natural Products to Drug Discovery in Tropical Diseases. In Comprehensive Analysis of Parasite Biology, From Metabolism to Drug Discovery; Müller, S., Cerdan, R., Radulescu, O., Eds.; Wiley-VCH: Weheim, Germany, 2016; pp. 75-104.

4. Shen, B. A new golden age of natural products drug discovery. Cell 2015, 163, 1297-1300. [CrossRef] [PubMed]

5. David, B.; Wolfender, J.L.; Dias, D.A. The pharmaceutical industry and natural products, historical status and new trends. Phytochem. Rev. 2015, 14, 299-315. [CrossRef]

6. Newman, D.J.; Cragg, G.M.; Snader, K.M. Natural products as sources of new drugs over the period 1981-2002. J. Nat. Prod. 2003, 66, 1022-1037. [CrossRef] [PubMed]

7. Koehn, F.E.; Carter, G.T. The evolving role of natural products in drug discovery. Nat. Rev. Drug Discov. 2005, 4, 206. [CrossRef]

8. Wolfender, J.L.; Queiroz, E.F. Chemical diversity of natural resources and the bioactivity of their constituents. CHIMIA Int. J. Chem. 2012, 66, 324-329. [CrossRef]

9. Kikuchi, H.; Kawai, K.; Nakashiro, Y.; Yonezawa, T.; Kawaji, K.; Kodama, E.N.; Oshima, Y. Construction of a meroterpenoid-like compounds library based on diversity-enhanced extracts. Chem. Eur. J. 2019, 25, 1106-1112. [CrossRef]

10. Cornforth, J.W. Terpenoid biosynthesis. Chem. Br. 1968, 4, 102-106.

11. Matsuda, Y.; Abe, I. Biosynthesis of fungal meroterpenoids. Nat. Prod. Rep. 2016, 33, 26-53. [CrossRef]

12. Geris, R.; Simpson, T.J. Meroterpenoids produced by fungi. Nat. Prod. Rep. 2009, 26, 1063-1094. [CrossRef]

13. Peng, X.; Qiu, M. Meroterpenoids from Ganoderma Species: A review of last five years. Nat. Prod. Bioprospect. 2018, 8, 137-149. [CrossRef]

14. Matsuda, Y.; Awakawa, T.; Abe, I. Reconstituted biosynthesis of fungal meroterpenoid andrastin A. Tetrahedron 2013, 69, 8199-8204. [CrossRef]

15. El-Elimat, T.; Raja, H.A.; Ayers, S.; Kurina, S.J.; Burdette, J.E.; Mattes, Z.; Sabatelle, R.; Bacon, J.W.; Colby, A.H.; Grinstaff, M.W. Meroterpenoids from Neosetophoma sp., A dioxa [4.3. 3] ropellane ring system, potent cytotoxicity, and prolific expression. Org. Lett. 2019, 21, 529-534. [CrossRef] 
16. Joung, E.-J.; Gwon, W.-G.; Shin, T.; Jung, B.-M.; Choi, J.; Kim, H.-R. Anti-inflammatory action of the ethanolic extract from Sargassum serratifolium on lipopolysaccharide-stimulated mouse peritoneal macrophages and identification of active components. J. Appl. Phycol. 2017, 29, 563-573. [CrossRef]

17. Seo, Y.; Park, K.E.; Kim, Y.A.; Lee, H.-J.; Yoo, J.-S.; Ahn, J.-W.; Lee, B.-J. Isolation of tetraprenyltoluquinols from the brown alga Sargassum thunbergii. Chem. Pharm. Bull. 2006, 54, 1730-1733. [CrossRef]

18. Kim, J.A.; Ahn, B.N.; Kong, C.S.; Kim, S.K. Protective effect of chromene isolated from Sargassum horneri against UV-A-induced damage in skin dermal fibroblasts. Exp. Dermatol. 2012, 21, 630-631. [CrossRef]

19. Kim, S.; Lee, M.-S.; Lee, B.; Gwon, W.-G.; Joung, E.-J.; Yoon, N.-Y.; Kim, H.-R. Anti-inflammatory effects of sargachromenol-rich ethanolic extract of Myagropsis myagroides on lipopolysaccharide-stimulated BV-2 cells. BMC Complement. Altern. Med. 2014, 14, 231. [CrossRef]

20. Jeon, Y.; Jung, Y.; Kim, M.C.; Kwon, H.C.; Kang, K.S.; Kim, Y.K.; Kim, S.-N. Sargahydroquinoic acid inhibits TNF $\alpha$-induced AP-1 and NF- $\mathrm{kB}$ signaling in HaCaT cells through PPAR $\alpha$ activation. Biochem. Biophys. Res. Commun. 2014, 450, 1553-1559. [CrossRef]

21. Joung, E.-J.; Lee, B.; Gwon, W.-G.; Shin, T.; Jung, B.-M.; Yoon, N.-Y.; Choi, J.-S.; Oh, C.W.; Kim, H.-R. Sargaquinoic acid attenuates inflammatory responses by regulating NF- $\mathrm{kB}$ and Nrf2 Pathways in lipopolysaccharide-stimulated RAW 264.7 cells. Int. Immunopharmacol. 2015, 29, 693-700. [CrossRef]

22. Oh, S.-J.; Joung, E.-J.; Kwon, M.-S.; Lee, B.; Utsuki, T.; Oh, C.-W.; Kim, H.-R. Anti-inflammatory effect of ethanolic extract of Sargassum serratifolium in lipopolysaccharide-stimulated BV2 microglial cells. J. Med. Food 2016, 19, 1023-1031. [CrossRef]

23. Gwon, W.-G.; Joung, E.-J.; Kwon, M.-S.; Lim, S.-J.; Utsuki, T.; Kim, H.-R. Sargachromenol protects against vascular inflammation by preventing TNF- $\alpha$-induced monocyte adhesion to primary endothelial cells via inhibition of NF- $\mathrm{KB}$ activation. Int. Immunopharmacol. 2017, 42, 81-89. [CrossRef]

24. Kwon, M.; Lim, S.-J.; Lee, B.; Shin, T.; Kim, H.-R. Ethanolic extract of Sargassum serratifolium inhibits adipogenesis in 3T3-L1 preadipocytes by cell cycle arrest. J. Appl. Phycol. 2018, 30, 559-568. [CrossRef]

25. Kim, S.-N.; Lee, W.; Bae, G.-U.; Kim, Y.K. Anti-diabetic and hypolipidemic effects of Sargassum yezoense in db/db mice. Biochem. Biophys. Res. Commun. 2012, 424, 675-680. [CrossRef]

26. De la Mare, J.-A.; Lawson, J.C.; Chiwakata, M.T.; Beukes, D.R.; Edkins, A.L.; Blatch, G.L. Quinones and halogenated monoterpenes of algal origin show anti-proliferative effects against breast cancer cells in vitro. Investig. N. Drugs 2012, 30, 2187-2200. [CrossRef]

27. Kang, C.-W.; Park, M.-S.; Kim, N.-H.; Lee, J.-H.; Oh, C.-W.; Kim, H.-R.; Kim, G.-D. Hexane extract from Sargassum serratifolium inhibits the cell proliferation and metastatic ability of human glioblastoma U87MG cells. Oncol. Rep. 2015, 34, 2602-2608. [CrossRef]

28. Tsang, C.K.; Kamei, Y. Sargaquinoic acid supports the survival of neuronal PC12D cells in a nerve growth factor-independent manner. Eur. J. Pharmacol. 2004, 488, 11-18. [CrossRef]

29. El-Demerdash, A.; Kumla, D.; Kijjoa, A. Chemical diversity and biological activities of meroterpenoids from marine derived-fungi: A comprehensive update. Mar. Drugs 2020, 18, 317. [CrossRef]

30. Zhao, M.; Tang, Y.; Xie, J.; Zhao, Z.; Cui, H. Meroterpenoids produced by fungi: Occurrence, structural diversity, biological activities, and their molecular targets. Eur. J. Med. Chem. 2021, 209, 112860. [CrossRef]

31. Gozari, M.; Alborz, M.; El-Seedi, R.H.; Reza, R.J. Chemistry, biosynthesis and biological activity of terpenoids and meroterpenoids in bacteria and fungi isolated from different marine habitats. Eur. J. Med. Chem. 2021, 210, 112957. [CrossRef]

32. Jiang, M.; Wu, Z.; Liu, L.; Chen, S. The chemistry and biology of fungal meroterpenoids (2009-2019). Org. Biomol. Chem. 2021, 19, 1644-1704. [CrossRef]

33. Qin, X.-J.; Yu, Q.; Yan, H.; Khan, A.; Feng, M.-Y.; Li, P.-P.; Hao, X.-J.; An, L.-K.; Liu, H.-Y. Meroterpenoids with antitumor activities from guava (Psidium guajava). J. Agric. Food Chem. 2017, 65, 4993-4999. [CrossRef] [PubMed]

34. Wang, Y.; Duan, M.; Zhao, L.; Ma, P. Guajadial inhibits NSCLC growth and migration following activation of the VEGF receptor-2. Fitoterapia 2018, 129, 73-77. [CrossRef] [PubMed]

35. Qin, X.-J.; Yan, H.; Ni, W.; Yu, M.-Y.; Khan, A.; Liu, H.; Zhang, H.-X.; He, L.; Hao, X.-J.; Di, Y.-T. Cytotoxic meroterpenoids with rare skeletons from Psidium guajava cultivated in temperate zone. Sci. Rep. 2016, 6, 32748. [CrossRef] [PubMed]

36. Shang, Z.C.; Yang, M.H.; Jian, K.L.; Wang, X.B.; Kong, L.Y. ${ }^{1}$ H NMR-guided isolation of formyl-phloroglucinol meroterpenoids from the leaves of Eucalyptus robusta. Chem. Eur. J. 2016, 22, 11778-11784. [CrossRef]

37. Farias, I.V.; Faqueti, L.G.; Noldin, V.F.; Junior, G.F.; Nowil, A.E.; Schuquel, I.T.; DelleMonache, F.; García, P.A.; López-Pérez, J.L.; SanFeliciano, A. Cytotoxic phloroglucinol meroterpenoid from Eugenia umbelliflora fruits. Phytochem. Lett. 2018, $27,187-192$. [CrossRef]

38. Liu, H.; Feng, M.-Y.; Yu, Q.; Yan, H.; Zeng, Y.; Qin, X.-J.; He, L.; Liu, H.-Y. Formyl phloroglucinol meroterpenoids from Eucalyptus tereticornis and their bioactivities. Tetrahedron 2018, 74, 1540-1545. [CrossRef]

39. Qin, X.-J.; Jin, L.-Y.; Yu, Q.; Liu, H.; Khan, A.; Yan, H.; Hao, X.-J.; An, L.-K.; Liu, H.-Y. Eucalypglobulusals A-J: Formylphloroglucinol-terpene meroterpenoids from Eucalyptus globulus fruits. J. Nat. Prod. 2018, 81, 2638-2646. [CrossRef]

40. Hu, L.; Xue, Y.; Zhang, J.; Zhu, H.; Chen, C.; Li, X.-N.; Liu, J.; Wang, Z.; Zhang, Y.; Zhang, Y. ( \pm )-Japonicols A-D: Acylphloroglucinol-based meroterpenoid enantiomers with anti-KSHV activities from Hypericum japonicum. J. Nat. Prod. 2016, 79, 1322-1328. [CrossRef]

41. Hu, L.; Liu, Y.; Wang, Y.; Wang, Z.; Huang, J.; Xue, Y.; Liu, J.; Liu, Z.; Chen, Y.; Zhang, Y. Discovery of acylphloroglucinol-based meroterpenoid enantiomers as KSHV inhibitors from Hypericum japonicum. RSC Adv. 2018, 8, 24101-24109. [CrossRef] 
42. Tang, G.-H.; Dong, Z.; Guo, Y.-Q.; Cheng, Z.-B.; Zhou, C.-J.; Yin, S. Psiguajadials A-K: Unusual Psidium meroterpenoids as phosphodiesterase-4 inhibitors from the leaves of Psidium guajava. Sci. Rep. 2017, 7, 1047. [CrossRef]

43. Shang, Z.-C.; Yang, M.-H.; Liu, R.-H.; Wang, X.-B.; Kong, L.-Y. New formyl phloroglucinol meroterpenoids from the leaves of Eucalyptus robusta. Sci. Rep. 2016, 6, 39815. [CrossRef]

44. Yu, Y.; Gan, L.-S.; Yang, S.-P.; Sheng, L.; Liu, Q.-F.; Chen, S.-N.; Li, J.; Yue, J.-M. Eucarobustols A-I: Conjugates of sesquiterpenoids and acylphloroglucinols from Eucalyptus robusta. J. Nat. Prod. 2016, 79, 1365-1372. [CrossRef]

45. Liu, J.; Jiang, L.-R.; Liu, M.-F. Unusual meroterpenoids from leaves of Psidium guajava. Chem. Nat. Compd. 2016, 52, 67-70. [CrossRef]

46. Qin, X.-J.; Feng, M.-Y.; Liu, H.; Ni, W.; Rauwolf, T.; Porco, J.A., Jr.; Yan, H.; He, L.; Liu, H.-Y. Eucalyptusdimers A-C: Dimeric phloroglucinol-phellandrene meroterpenoids from Eucalyptus robusta. Org. Lett. 2018, 20, 5066-5070. [CrossRef]

47. Qin, X.-J.; Liu, H.; Yu, Q.; Yan, H.; Tang, J.-F.; An, L.-K.; Khan, A.; Chen, Q.-R.; Hao, X.-J.; Liu, H.-Y. Acylphloroglucinol derivatives from the twigs and leaves of Callistemon salignus. Tetrahedron 2017, 73, 1803-1811. [CrossRef]

48. Chen, N.-H.; Zhang, Y.-B.; Huang, X.-J.; Jiang, L.; Jiang, S.-Q.; Li, G.-Q.; Li, Y.-L.; Wang, G.-C. Drychampones A-C: Three meroterpenoids from Dryopteris championii. J. Org. Chem. 2016, 81, 9443-9448. [CrossRef]

49. Li, C.-J.; Ma, J.; Sun, H.; Zhang, D.; Zhang, D.-M. Guajavadimer A, a dimeric caryophyllene-derived meroterpenoid with a new carbon skeleton from the leaves of Psidium guajava. Org. Lett. 2016, 18, 168-171. [CrossRef]

50. Chen, N.; Wu, Z.; Li, W.; Li, Y.; Luo, D.; Chen, L.; Zhang, X.; Zhang, Y.; Wang, G.; Li, Y. Acylphloroglucinols-based meroterpenoid enantiomers with antiviral activities from Dryopteris crassirhizoma. Ind. Crops Prod. 2020, 150, 112415. [CrossRef]

51. Hou, J.-Q.; Fan, C.-L.; Pei, X.; Hang, P.-L.; Deng, F.; Jiang, W.-Q.; Wang, G.-C.; Zhang, X.-Q.; Ye, W.-C.; Wang, H. Psiguadiols A-J, rearranged meroterpenoids as potent PTP1B inhibitors from Psidium guajava. J. Nat. Prod. 2019, 82, 3267-3278. [CrossRef]

52. Xu, J.; Zhu, H.-L.; Zhang, J.; Liu, W.-Y.; Luo, J.-G.; Pan, K.; Cao, W.-Y.; Bi, Q.-R.; Feng, F.; Qu, W. Littordials A-E, novel formyl-phloroglucinol- $\beta$-caryophyllene meroterpenoids from the leaves of Psidium littorale. Org. Chem. Front. 2019, 6, 1667-1673. [CrossRef]

53. Shang, Z.-C.; Han, C.; Xu, J.-L.; Liu, R.-H.; Yin, Y.; Wang, X.-B.; Yang, M.-H.; Kong, L.-Y. Twelve formyl phloroglucinol meroterpenoids from the leaves of Eucalyptus robusta. Phytochemistry 2019, 163, 111-117. [CrossRef]

54. Xie, X.; Wu, L.; Cui, Z.; Yang, M.; Yin, Y.; Luo, J.; Kong, L. Melaleucadines A and B: Two rare benzylic phloroglucinol-terpene hybrids from Melaleuca leucadendron. Tetrahedron Lett. 2019, 60, 1011-1013. [CrossRef]

55. Zhu, H.-L.; Hu, Y.-W.; Qu, W.; Zhang, J.; Guo, E.-Y.; Jiang, X.-Y.; Liu, W.-Y.; Feng, F.; Xu, J. Littordial F, a novel phloroglucinol meroterpenoid from the Leaves of Psidium littorale. Tetrahedron Lett. 2019, 60, 1868-1870. [CrossRef]

56. Zhang, J.; He, J.; Cheng, Y.-C.; Zhang, P.-C.; Yan, Y.; Zhang, H.-J.; Zhang, W.-K.; Xu, J.-K. Fischernolides A-D, four novel diterpene-based eroterpenoid scaffolds with antitumor activities from Euphorbia fischeriana. Org. Chem. Front. 2019, 6, $2312-2318$. [CrossRef]

57. Xie, R.; Li, L.; Fan, X.; Zi, J. Euphoractone, a cytotoxic meroterpenoid with an unusual ent-abietane-phloroglucinol skeleton, from Euphorbia fischeriana Steud. Chin. Chem. Lett. 2020, 31, 431-433. [CrossRef]

58. Qin, X.-J.; Zhi, Y.-E.; Yan, H.; Zhang, Y.; Liu, H.; Yu, Q.; Wang, S.; Zhao, Q.; He, L.; Ma, X. Baeckfrutones A-L: Polymethylated phloroglucinol meroterpenoids from the twigs and leaves of Baeckea frutescens. Tetrahedron 2018, 74, 6658-6666. [CrossRef]

59. Zhi, Y.-E.; Qi, X.-J.; Liu, H.; Zeng, Y.; Ni, W.; He, L.; Wang, Z.-D.; Liu, H.-Y. Structurally diverse polymethylated phloroglucinol meroterpenoids from Baeckea frutescens. Nat. Products Bioprospect. 2018, 8, 431-439. [CrossRef]

60. Wu, R.; Le, Z.; Wang, Z.; Tian, S.; Xue, Y.; Chen, Y.; Hu, L.; Zhang, Y. Hyperjaponol H: A new bioactive filicinic acid-based meroterpenoid from Hypericum japonicum Thunb. ex Murray. Molecules 2018, 23, 683. [CrossRef] [PubMed]

61. Liu, H.-X.; Zhang, W.-M.; Xu, Z.-F.; Chen, Y.-C.; Tan, H.-B.; Qiu, S.-X. Isolation, synthesis, and biological activity of tomentosenol A from the leaves of Rhodomyrtus tomentosa. RSC Adv. 2016, 6, 25882-25886. [CrossRef]

62. Liu, C.; Ang, S.; Huang, X.-J.; Tian, H.-Y.; Deng, Y.-Y.; Zhang, D.-M.; Wang, Y.; Ye, W.-C.; Wang, L. Meroterpenoids with new skeletons from Myrtus communis and structure revision of myrtucommulone K. Org. Lett. 2016, 18, 4004-4007. [CrossRef] [PubMed]

63. Cottiglia, F.; Casu, L.; Leonti, M.; Caboni, P.; Floris, C.; Busonera, B.; Farci, P.; Ouhtit, A.; Sanna, G. Cytotoxic phloroglucinols from the leaves of Myrtus communis. J. Nat. Prod. 2012, 75, 225-229. [CrossRef]

64. Hou, J.-Q.; Guo, C.; Zhao, J.-J.; He, Q.-W.; Zhang, B.-B.; Wang, H. Frutescone A-G: Tasmanone-based meroterpenoids from the aerial parts of Baeckea frutescens. J. Org. Chem. 2017, 82, 1448-1457. [CrossRef]

65. Zhang, Y.-L.; Chen, C.; Wang, X.-B.; Wu, L.; Yang, M.-H.; Luo, J.; Zhang, C.; Sun, H.-B.; Luo, J.-G.; Kong, L.-Y. Rhodomyrtials A and B: Two meroterpenoids with a triketone-sesquiterpene-triketone skeleton from Rhodomyrtus tomentosa, structural elucidation and biomimetic synthesis. Org. Lett. 2016, 18, 4068-4071. [CrossRef]

66. Zhang, Y.-L.; Zhou, X.-W.; Wu, L.; Wang, X.-B.; Yang, M.-H.; Luo, J.; Luo, J.-G.; Kong, L.-Y. Isolation, structure elucidation, and absolute configuration of syncarpic acid-conjugated terpenoids from Rhodomyrtus tomentosa. J. Nat. Prod. 2017, 80, 989-998. [CrossRef]

67. Qin, X.-J.; Shu, T.; Yu, Q.; Yan, H.; Ni, W.; An, L.-K.; Li, P.-P.; Zhi, Y.-E.; Khan, A.; Liu, H.-Y. Cytotoxic acylphloroglucinol derivatives from Callistemon salignus. Nat. Prod. Bioprospect. 2017, 7, 315-321. [CrossRef]

68. Hou, J.-Q.; Guo, C.; Zhao, J.-J.; Dong, Y.-Y.; Hu, X.-L.; He, Q.-W.; Zhang, B.-B.; Yan, M.; Wang, H. Anti-inflammatory meroterpenoids from Baeckea frutescens. J. Nat. Prod. 2017, 80, 2204-2214. [CrossRef] 
69. Hou, J.-Q.; Wang, B.-L.; Han, C.; Xu, J.; Wang, Z.; He, Q.-W.; Zhang, P.-L.; Zhao, S.-M.; Pei, X.; Wang, H. Atropisomeric meroterpenoids with rare triketone-phloroglucinol-terpene hybrids from Baeckea frutescens. Org. Biomol. Chem. 2018, 16, 8513-8524. [CrossRef]

70. Hu, L.; Zhang, Y.; Zhu, H.; Liu, J.; Li, H.; Li, X.-N.; Sun, W.; Zeng, J.; Xue, Y.; Zhang, Y. Filicinic acid based meroterpenoids with anti-epstein-barr virus activities from Hypericum japonicum. Org. Lett. 2016, 18, 2272-2275. [CrossRef]

71. Zhang, H.-X.; Lunga, P.K.; Li, Z.J.; Dai, Q.; Du, Z.-Z. Flavonoids and stilbenoids from Derris eriocarpa. Fitoterapia 2014, 95, 147-153. [CrossRef]

72. Chen, M.; Chen, L.-F.; Li, M.-M.; Li, N.-P.; Cao, J.-Q.; Wang, Y.; Li, Y.-L.; Wang, L.; Ye, W.-C. Myrtucomvalones A-C, three unusual triketone-Sesquiterpene adducts from the leaves of Myrtus communis 'Variegata'. RSC Adv. 2017, 7, 22735-22740. [CrossRef]

73. Liu, H.-X.; Chen, K.; Yuan, Y.; Xu, Z.-F.; Tan, H.-B.; Qiu, S.-X. Rhodomentones A and B, novel meroterpenoids with unique NMR characteristics from Rhodomyrtus tomentosa. Org. Biomol. Chem. 2016, 14, 7354-7360. [CrossRef] [PubMed]

74. Senadeera, S.P.D.; Robertson, L.P.; Duffy, S.; Wang, Y.; Avery, V.M.; Carroll, A.R. $\beta$-Triketone-monoterpene mybrids from the flowers of the australian tree Corymbia intermedia. J. Nat. Prod. 2018, 81, 2455-2461. [CrossRef]

75. Li, Q.-J.; Tang, P.-F.; Zhou, X.; Lu, W.-J.; Xu, W.-J.; Luo, J.; Kong, L.-Y. Dimethylated acylphloroglucinol meroterpenoids with anti-oral-bacterialand anti-inflammatory activities from Hypericum elodeoides. Bioorgan. Chem. 2020, 104, 104275. [CrossRef] [PubMed]

76. Liu, H.; Li, P.; Bi, L.-S.; Wu, W.-J.; Yan, H.; He, L.; Qin, X.-J.; Liu, H.-Y. Polymethylated phloroglucinol meroterpenoids from Rhodomyrtus tomentosa and their antibacterial and acetylcholinesterase inhibitory effects. Chem. Biodivers. 2020, 17, e2000489. [CrossRef] [PubMed]

77. Hou, J.-Q.; Zhao, H.; Yu, J.-H.; Chen, L.-F.; Hao, W. New meroterpenoids and C-methylated flavonoid isolated from Baeckea frutescens. Chin. J. Nat. Med. 2020, 18, 379-384. [CrossRef]

78. Deng, X.; Wang, X.; Wu, L. Triketone-terpene meroterpenoids from the leaves of Rhodomyrtus tomentosa. Fitoterapia 2020, 143, 104585. [CrossRef] [PubMed]

79. Asolkar, R.N.; Singh, A.; Jensen, P.R.; Aalbersberg, W.; Carte, B.K.; Feussner, K.-D.; Subramani, R.; DiPasquale, A.; Rheingold, A.L.; Fenical, W. Marinocyanins: Cytotoxic bromo-phenazinone meroterpenoids from a marine bacterium from the Streptomycete clade MAR4. Tetrahedron 2017, 73, 2234-2241. [CrossRef] [PubMed]

80. Kohatsu, H.; Kamo, S.; Tomoshige, S.; Kuramochi, K. Total syntheses of pyocyanin, lavanducyanin, and marinocyanins A and B. Org. Lett. 2019, 21, 7311-7314. [CrossRef]

81. Zhang, X.; Li, P.-L.; Qin, G.-F.; Li, S.; de Voogd, N.J.; Tang, X.-L.; Li, G.-Q. Isolation and absolute configurations of diversiform C17, C21 and C25 terpenoids from the marine Sponge Cacospongia sp. Mar. Drugs 2019, 17, 14. [CrossRef]

82. Nguyen, H.M.; Ito, T.; Win, N.N.; Kodama, T.; Hung, V.Q.; Nguyen, H.T.; Morita, H. New antibacterial sesquiterpene aminoquinones from a vietnamese marine sponge of Spongia sp. Phytochem. Lett. 2016, 17, 288-292. [CrossRef]

83. Nguyen, H.M.; Ito, T.; Kurimoto, S.-I.; Ogawa, M.; Win, N.N.; Hung, V.Q.; Nguyen, H.T.; Kubota, T.; Kobayashi, J.I.; Morita, H. New merosesquiterpenes from a Vietnamese marine sponge of Spongia sp. and their biological activities. Bioorg. Med. Chem. Lett. 2017, 27, 3043-3047. [CrossRef]

84. Zhang, X.; Xu, H.-Y.; Huang, A.-M.; Wang, L.; Wang, Q.; Cao, P.-Y.; Yang, P.-M. Antibacterial meroterpenoids from the south China sea sponge Dysidea sp. Chem. Pharm. Bull. 2016, 64, 1036-1042. [CrossRef]

85. Li, J.; Yang, F.; Wang, Z.; Wu, W.; Liu, L.; Wang, S.-P.; Zhao, B.-X.; Jiao, W.-H.; Xu, S.-H.; Lin, H.-W. Unusual anti-inflammatory meroterpenoids from the marine sponge Dactylospongia sp. Org. Biomol. Chem. 2018, 16, 6773-6782. [CrossRef]

86. Oba, Y.; Yoshida, N.; Kanie, S.; Ojika, M.; Inouye, S. Biosynthesis of firefly luciferin in adult lantern, decarboxylation of L-cysteine is a key step for benzothiazole ring formation in firefly luciferin synthesis. PLoS ONE 2013, 8, e84023. [CrossRef]

87. Wang, J.; Mu, F.-R.; Jiao, W.-H.; Huang, J.; Hong, L.-L.; Yang, F.; Xu, Y.; Wang, S.-P.; Sun, F.; Lin, H.-W. Meroterpenoids with protein tyrosine phosphatase $1 \mathrm{~b}$ inhibitory activity from a Hyrtios sp. marine sponge. J. Nat. Prod. 2017, 80, 2509-2514. [CrossRef]

88. Jiao, W.-H.; Cheng, B.-H.; Shi, G.-H.; Chen, G.-D.; Gu, B.-B.; Zhou, Y.-J.; Hong, L.-L.; Yang, F.; Liu, Z.-Q.; Qiu, S.-Q. Dysivillosins A-D: Unusual anti-allergic meroterpenoids from the marine sponge Dysidea villosa. Sci. Rep. 2017, 7, 8947. [CrossRef]

89. Salame, R.; Gravel, E.; Leblanc, K.; Poupon, E. Biomimetic synthesis of tangutorine following new biogenetic proposals. Org. Lett. 2009, 11, 1891-1894. [CrossRef]

90. Liu, D.; Li, Y.; Li, X.; Cheng, Z.; Huang, J.; Proksch, P.; Lin, W. Chartarolides A-C: Novel meroterpenoids with antitumor activities. Tetrahedron Lett. 2017, 58, 1826-1829. [CrossRef]

91. Jiao, W.-H.; Cheng, B.-H.; Chen, G.-D.; Shi, G.-H.; Li, J.; Hu, T.-Y.; Lin, H.-W. Dysiarenone, a dimeric C21 meroterpenoid with inhibition of COX-2 expression from the marine sponge Dysidea arenaria. Org. Lett. 2018, 20, 3092-3095. [CrossRef]

92. Rehman, N.; Hussain, H.; Al-Shidhani, S.; Avual, S.K.; Abbas, G.; Anwar, M.U.; Górecki, M.; Pescitelli, G.; Al-Harrasi, A. Incensfuran: Isolation, $\mathrm{X}$-ray crystal structure and absolute configuration by means of chiroptical studies in solution and solid state. RSC Adv. 2017, 7, 42357-42362. [CrossRef]

93. Jiao, W.-H.; Shi, G.-H.; Xu, T.-T.; Chen, G.-D.; Gu, B.-B.; Wang, Z.; Peng, S.; Wang, S.-P.; Li, J.; Han, B.-N.; et al. Dysiherbols A-C and dysideanone E, cytotoxic and NF- $\mathrm{kB}$ inhibitory tetracyclic meroterpenes from a Dysidea sp. marine sponge. J. Nat. Prod. 2016, 79, 406-411. [CrossRef]

94. Jiao, W.-H.; Li, J.; Wang, D.; Zhang, M.-M.; Liu, L.-Y.; Sun, F.; Li, J.Y.; Capon, R.J.; Lin, H.-W. Cinerols, nitrogenous meroterpenoids from the marine sponge Dysidea cinerea. J. Nat. Prod. 2019, 82, 2586-2593. [CrossRef] 
95. Le, T.C.; Lee, E.J.; Lee, J.; Hong, A.; Yim, C.-Y.; Yang, I.; Choi, H.; Chin, J.; Cho, S.J.; Ko, J.; et al. Saccharoquinoline, a cytotoxic alkaloidal meroterpenoid from marine-derived bacterium Saccharomonospora sp. Mar. Drugs 2019, 17, 98. [CrossRef]

96. Yu, H.-B.; Yin, Z.-F.; Gu, B.-N.; Zhang, J.-P.; Wang, S.-P.; Yang, F.; Lin, H.-W. Cytotoxic meroterpenoids from the marine sponge Dactylospongia elegans. Nat. Prod. Res. 2021. in print. [CrossRef]

97. Gui, Y.-H.; Jiao, W.-H.; Zhou, M.; Zhang, Y.; Zeng, D.-Q.; Zhu, H.-R.; Liu, K.-C.; Sun, F.; Chen, H.-F.; Lin, H.W. Septosones A-C, in vivo anti-inflammatory meroterpenoids with rearranged carbon skeletons from the marine sponge Dysidea septosa. Org. Lett. 2019, 21, 767-770. [CrossRef]

98. Hamed, A.; Abdel-Razek, A.; Frese, M.; Stammler, H.; El-Haddad, A.; Ibrahim, T.; Sewald, N.; Shaaban, M.; Terretonin, N. A new meroterpenoid from Nocardiopsis sp. Molecules 2018, 23, 299. [CrossRef]

99. Choi, H.; Hwang, H.; Chin, J.; Kim, E.; Lee, J.; Nam, S.-J.; Lee, B.C.; Rho, B.J.; Kang, H. Tuberatolides, potent fxr antagonists from the korean marine tunicate Botryllus tuberatus. J. Nat. Prod. 2011, 74, 90-94. [CrossRef]

100. Choi, Y.; Kim, J.; Lee, K.; Choi, Y.-J.; Ye, B.-R.; Kim, M.-S.; Ko, S.-G.; Lee, S.-H.; Kang, D.-H.; Heo, S.-J. Tuberatolide B suppresses cancer progression by promoting ROS-mediated inhibition of STAT3 signaling. Mar. Drugs 2017, 15, 55. [CrossRef]

101. Yang, Y.-X.; Wang, J.-X.; Wang, Q.; Li, H.-I.; Tao, M.; Luo, Q.; Liu, H. New chromane and chromene meroterpenoids from flowers of Rhododendron rubiginosum Franch. var. rubiginosum. Fitoterapia 2018, 127, 396-401. [CrossRef]

102. Hanuš, L.O.; Meyer, S.M.; Muñoz, E.; Taglialatela-Scafati, O.; Appendino, G. Phytocannabinoids, a unified critical inventory. Nat. Prod. Rep. 2016, 33, 1357-1392. [CrossRef]

103. Kang, H.-S.; Kim, J.-P. New chromene derivatives with radical scavenging activities from the brown alga Sargassum siliquastrum. J. Chem. Res. 2017, 41, 116-119. [CrossRef]

104. Liao, H.-B.; Huang, G.-H.; Yu, M.-H.; Lei, C.; Hou, A.-J. Five pairs of meroterpenoid enantiomers from Rhododendron capitatum. J. Org. Chem. 2017, 82, 1632-1637. [CrossRef] [PubMed]

105. Huang, G.-H.; Hu, Z.; Lei, C.; Wang, P.-P.; Yang, J.; Li, J.-Y.; Li, J.; Hou, A.-J. Enantiomeric pairs of meroterpenoids with diverse heterocyclic systems from Rhododendron nyingchiense. J. Nat. Prod. 2018, 81, 1810-1818. [CrossRef] [PubMed]

106. Seong, S.H.; Ali, M.Y.; Kim, H.-R.; Jung, H.A.; Choi, J.S. BACE1 inhibitory activity and molecular docking analysis of meroterpenoids from Sargassum serratifolium. Bioorg. Med. Chem. 2017, 25, 3964-3970. [CrossRef] [PubMed]

107. De Sousa, C.B.; Gangadhar, K.N.; Morais, T.R.; Conserva, G.A.; Vizetto-Duarte, C.; Pereira, H.; Laurenti, M.D.; Campino, L.; Levy, D.; Uemi, M. Antileishmanial activity of meroditerpenoids from the macroalgae Cystoseira baccata. Exp. Parasitol. 2017, 174, 1-9. [CrossRef] [PubMed]

108. Chiou, C.-T.; Shen, C.-C.; Tsai, T.-H.; Chen, Y.-J.; Lin, L.-C. Meroterpenoids and chalcone-lignoids from the roots of Mimosa diplotricha. J. Nat. Prod. 2016, 79, 2439-2445. [CrossRef] [PubMed]

109. Yang, W.-Q.; Hai, P.; Xiao, H.; Gao, Y.; Tao, Y.; Miao, D.; Wang, F. Glabralides A-C, three novel meroterpenoids from Sarcandra glabra. Tetrahedron 2018, 74, 341-347. [CrossRef]

110. Huang, G.-H.; Lei, C.; Zhu, K.-X.; Li, J.-Y.; Li, J.; Hou, A.-J. Enantiomeric pairs of meroterpenoids from Rhododendron fastigiatum. Chin. J. Nat. Med. 2019, 17, 963-969. [CrossRef]

111. Shi, Q.; Li, F.-T.; Wu, Y.-M.; Sun, X.-Y.; Lei, C.; Li, J.-Y.; Hou, A.-J. Meroterpenoids with diverse structures and anti-inflammatory activities from Rhododendron anthopogonoides. Phytochemistry 2020, 180, 112524. [CrossRef]

112. Xu, Q.-X.; Zhang, Y.-B.; Liu, X.-Y.; Xu, W.; Yang, X.-W. Cytotoxic heterodimers of meroterpene phenol from the fruits of Psoralea Corylifolia. Phytochemistry 2020, 176, 112394. [CrossRef]

113. Ni, G.; Shi, G.-R.; Li, J.-Y.; Yu, D.-Q. The unprecedented iridal lactone and adducts of spiroiridal and isoflavonoid from Belamcanda chinensis. RSC Adv. 2017, 7, 20160-20166. [CrossRef]

114. Zhu, Y.-D.; Chen, R.-C.; Wang, H.; Jiang, H.; Huang, X.-L.; Zhang, M.-L.; Li, L.-Y.; Hu, Z.; Xu, X.-D.; Wang, C.-J. Two new flavonoidtriterpene saponin meroterpenoids from Clinopodium chinense and their protective effects against anoxia/reoxygenation-induced apoptosis in H9c2 cells. Fitoterapia 2018, 128, 180-186. [CrossRef]

115. Martucci, H.; Campit, S.E.; Gee, S.R.; Bray, W.M.; Gokey, T.; Cada, A.K.; Yen, T.-Y.; Minoura, K.; Guliaev, A.B.; Lokey, R.S. Naphthablins B and C: Meroterpenoids identified from the marine sediment-derived Streptomyces sp. CP26-58 using HeLa cell-based cytological profiling. J. Nat. Prod. 2017, 80, 684-691. [CrossRef]

116. Li, C.; Li, C.-J.; Ma, J.; Chen, F.-Y.; Li, L.; Wang, X.-L.; Ye, F.; Zhang, D.-M. Magterpenoids A-C: Three polycyclic meroterpenoids with PTP1B inhibitory activity from the bark of Magnolia officinalis var. biloba. Org. Lett. 2018, 20, 3682-3686. [CrossRef]

117. Wang, Y.; Zhu, Y.; Xiao, L.; Ge, L.; Wu, X.; Wu, W.; Wan, H.; Zhang, K.; Li, J.; Zhou, B. Meroterpenoids isolated from Arnebia euchroma (Royle) Johnst. and their cytotoxic activity in human hepatocellular carcinoma cells. Fitoterapia 2018, 131, 236-244. [CrossRef]

118. Wan, H.; Li, J.; Zhang, K.; Zou, X.; Ge, L.; Zhu, F.; Zhou, H.; Gong, M.; Wang, T.; Chen, D. A new meroterpenoid functions as an anti-tumor agent in hepatoma cells by downregulating mTOR activation and inhibiting EMT. Sci. Rep. 2018, 8, 13152. [CrossRef]

119. Lai, K.-H.; Liu, Y.-C.; Su, J.-H.; El-Shazly, M.; Wu, C.-F.; Du, Y.-C.; Hsu, Y.-M.; Yang, J.-C.; Weng, M.-K.; Chou, C.-H. Antileukemic scalarane sesterterpenoids and meroditerpenoid from Carteriospongia (Phyllospongia) sp., induce apoptosis via dual inhibitory effects on topoisomerase II and Hsp90. Sci. Rep. 2016, 6, 36170. [CrossRef]

120. Shen, X.; Wang, X.; Huang, T.; Deng, Z.; Lin, S. Naphthoquinone-based meroterpenoids from marine-derived streptomyces sp. B9173. Biomolecules 2020, 10, 1187. [CrossRef] 
121. Ryu, M.-J.; Hwang, S.; Kim, S.; Yang, I.; Oh, D.-C.; Nam, S.-J.; Fenical, W. Meroindenon and merochlorins E and F, antibacterial meroterpenoids from a marine-derived sediment bacterium of the Genus Streptomyces. Org. Lett. 2019, 21, 5779-5783. [CrossRef]

122. Joy, M.; Chakraborty, K. Specialized oxygenated heterocyclics from Villorita cyprinoides with cyclooxygenase-2 and 5-lipoxygenase inhibitory properties. Food Res. Int. 2018, 106, 164-172. [CrossRef]

123. Joy, M.; Chakraborty, K. Antioxidative and anti-inflammatory pyranoids and isochromenyl analogues from Corbiculid bivalve clam, Villorita cyprinoides. Food Chem. 2018, 251, 125-134. [CrossRef]

124. Chakraborty, K.; Joseph, D.; Joy, M.; Raola, V.K. Characterization of substituted aryl meroterpenoids from red seaweed Hypnea musciformis as potential antioxidants. Food Chem. 2016, 212, 778-788. [CrossRef]

125. De los Reyes, C.; Ortega, M.J.; Zbakh, H.; Motilva, V.; Zubía, E. Cystoseira usneoides, A brown alga rich in antioxidant and anti-inflammatory meroditerpenoids. J. Nat. Prod. 2016, 79, 395-405. [CrossRef]

126. Joy, M.; Chakraborty, K. First report of two new antioxidative meroterpeno $2 \mathrm{H}$-pyranoids from short-necked yellow-foot clam Paphia malabarica (family, Veneridae) with bioactivity against pro-inflammatory cyclooxygenases and lipoxygenase. Nat. Prod. Res. 2016, 31, 615-625. [CrossRef] [PubMed]

127. Wang, X.; Li, L.; Zhu, R.; Zhang, J.; Zhou, J.; Lou, H. Bibenzyl-based meroterpenoid enantiomers from the Chinese liverwort Radula sumatrana. J. Nat. Prod. 2017, 80, 3143-3150. [CrossRef]

128. Jiaa, X.; Wu, Y.; Lei, C.; Yu, Y.; Li, J.; Li, J.; Hou, A. Hyperinoids A and B, two polycyclic meroterpenoids from Hypericum patulum. Chin. Chem. Lett. 2020, 31, 1263-1266. [CrossRef]

129. Wang, S.-X.; Zhao, R.-L.; Guo, C.; Chen, B.-S.; Dai, H.-Q.; Liu, G.-Q.; Liu, H.-W. New meroterpenoid compounds from the culture of mushroom Panus lecomtei. Chin. J. Nat. Med. 2020, 18, 268-272. [CrossRef]

130. Day, A.J.; George, J.H. Isolation and biomimetic oxidation of prenylbruceol A, an anticipated meroterpenoid natural product from Philotheca myoporoides. J. Nat. Prod. 2020, 83, 2305-2309. [CrossRef]

131. Gurr, J.R.; O’Donnell, T.J.; Luo, Y.; Yoshida, W.Y.; Hall, M.L.; Mayer, A.M.S.; Sun, R.; Williams, P.G. 6-Deoxy- and 11hydroxytolypodiols: Meroterpenoids from the cyanobacterium HT-58-2. J. Nat. Prod. 2020, 83, 1691-1695. [CrossRef]

132. Zong, J.-F.; Hu, Z.; Shao, Y.-Y.; Shi, Q.; Zhang, M.-M.; Zhou, Y.-B.; Li, J.; Hou, A.J. Hyperprins A and B, two complex meroterpenoids from Hypericum przewalskii. Org. Lett. 2020, 22, 2797-2800. [CrossRef]

133. Ye, Y.-S.; Du, S.-Z.; Jiang, N.-N.; Xu, H.-X.; Yang, J.; Fu, W.-W.; Nian, Y.; Xu, G. Novel meroterpenoids from Hypericum patulum: Highly potent late Nav1.5 sodium current inhibitors. Org. Lett. 2020, 22, 6339-6343. [CrossRef]

134. Nguyen, N.Y.T.; Pham, N.S.L.; Dang, P.H.; Huu, D.M.N.; Dang, H.P.; Tran, Q.L. Two new meroterpenoids from the aerial parts of Ampelopsis cantoniensis (Vitaceae). J. Asian Nat. Prod. Res. 2020, 22, 1152-1158. [CrossRef]

135. Wang, D.; Xiu, M.-X.; Li, H.-Z.; Xiong, D.-X.; Lee, H.-X.; Sun, Y.-N.; Cui, L. Two new meroterpenes with activity against diacylglycerol acyltransferase from seeds of Psoralea corylifolia. Phytochem. Lett. 2020, 40, 171-175. [CrossRef]

136. Xu, Q.-X.; Xu, W.; Yang, X.-W. Meroterpenoids from the fruits of Psoralea corylifolia. Tetrahedron 2020, 76, 131343. [CrossRef] 